\title{
Central Bank Intervention and the Intraday Process of Price Formation in the Currency Markets
}

\author{
Paolo Pasquariello ${ }^{1}$
}

October 4, 2002

1 Please address comments to the author at the Leonard N. Stern School of Business, New York University, Kaufman Management Education Center, Suite 9-180, 44 West 4th Street, New York, NY 10012-1126, or through email: ppasquar@stern.nyu.edu. This work has benefited from the encouragement and suggestions of Joel Hasbrouck and Marti Subrahmanyam, and from the comments of Viral Acharya, Deepak Agrawal, Yakov Amihud, Menachem Brenner, José Campa, Monique Ebell, Massimo Guidolin, Malcolm Harris, Jerry Kallberg, Richard Lyons, Arun Muralidhar, Gideon Saar, Alex Shapiro, William Silber, Raghu Sundaram, and other participants at the Stern Finance Seminar, the 2001 EFMA Doctoral Symposium, the 2001 EFA Meeting, the 2001 FMA Meeting, and the 2002 MFA Meeting. Any remaining errors are naturally my own. 


\begin{abstract}
We study the impact of sterilized Central Bank interventions on the microstructure of currency markets. We analyze their major channels of effectiveness, imperfect substitutability and signaling, in a model of sequential trading in which the stylized monetary authority is a rational, but not necessarily profit-maximizing player. In such a setting, and consistent with available empirical evidence, we find that intervention has endogenous long-lived effects on quotes when informative about policy objectives and fundamentals, or when the threat of future actions by the Central Bank is significant and credible, for these circumstances lead uninformed investors or dealers to a permanent revision in their beliefs. Portfolio balance effects of such transactions are instead short-lived, because of trading occurring sequentially and not because of high asset substitutability, as often argued in the literature. We also find that a monetary authority attempting to lean against the wind or to chase the trend of the domestic currency is generally more successful when dealers compete against each other for the incoming trades. Intuitively, competition induces the dealers to pass all revenues or losses they expect from trading with the Central Bank onto the population of investors. This is accomplished by greater, and generally asymmetric, revisions of their bid and ask quotes. The resulting equilibrium process of intraday price formation and bid-ask spreads are shown to depend crucially on the degree of market power held by the forex dealers, on the sign and magnitude of announced and realized interventions, on the perceived likelihood of a future intervention to occur, and on the transparency of the order flow induced by the intervention.
\end{abstract}




\section{Introduction and motivation}

The foreign exchange market is probably the most active financial market in the world in terms of volume, frequency, and intensity of trading. ${ }^{1}$ Nonetheless, constraints to the availability of data on intraday quotes, spreads, and transactions prevented investigations of the microstructure of currency markets from occurring with the same degree of detail as in the case of centralized equity markets like the NYSE. These constraints arise from the fact that currencies are traded over-the counter (OTC), hence banks and other dealers have no obligation to report their activity to the public. However, progress in computer and communications technology over the past few years has made live trading data available at shorter time intervals. A renewed interest in analyzing the microstructure of the foreign exchange (forex) market has ensued. In most of these cases however, with the notable exceptions of Lyons (1997), Naranjo and Nimalendran (2000), and Evans and Lyons (2001), researchers' efforts have been typically oriented toward applying and testing existing microstructure theories. Indeed, the empirical analysis of these rich datasets has not been preceded by theoretical studies of how institutional features specific to currency markets may affect their functioning and the resulting process of price formation. The main objective of this paper is to attempt to fill this gap. We focus on some important aspects of the global currency markets that are not shared by any equity market, in particular the likelihood of intervention by a rational, but not necessarily profit-maximizing player like the Central Bank $(\mathrm{CB})$, and derive from their inclusion several empirically testable implications for quotes and bid-ask spreads.

Many macroeconomics textbooks describe the exchange rate as an intermediate target of monetary policy. This simply means that CBs choose specific levels (or bands of fluctuation) for the domestic currency that are compatible with the "ultimate" trade-off of monetary policy, between a sustainable growth of the economy (the "output gap") and moderate levels of inflation. Each monetary authority weighs growth and price stability differently, but all of them are expected to participate in the currency markets to serve their agenda of political economy. CBs may also promote less stringent economic agendas, under pressures from political power, interest lobbies, etc. Nonetheless, in both cases the resulting actions by the CB may not be motivated by pure profit. Indeed, there is much anecdotal and empirical evidence that policy objectives and maximization of long-term wealth or short-term profit often collide. For example, Taylor (1982) uses the profit criterion of Friedman (1953) to show that, during the 1970s, CBs obtained only mixed results when actively resisting currency fluctuations, but lost billions of dollars in the process.

In the market microstructure literature on asymmetric information, insiders always experience profits at the expense of market-makers and uninformed (noise) traders. ${ }^{2}$ Forex dealers may instead reasonably expect not only to suffer potential losses but also to earn potential gains from trading against the government, even if the domestic monetary authorities are better informed about economic fundamentals and policy objectives. This again might occur when CB actions are rational, but not consistent with trading profit or wealth maximization. The market power held by dealers in the currency market would then determine how much of these potential gains

\footnotetext{
${ }^{1}$ The BIS (1999) triennial survey of currency market activity for 1998 reports that about $\$ 1.5$ trillion in transactions are executed in the global foreign exchange market every trading day.

${ }^{2}$ O'Hara (1995) reviews the market microstructure literature dealing with issues associated with information economics.
} 
or losses will be passed to investors via quotes' revisions and spreads' adjustments. CBs may also take positions in the currency markets following purely speculative motives, as in the frequently cited example of Bank Negara, the Malaysian monetary authority, in the early 1990s. ${ }^{3}$ In such circumstances, currency dealers may incur losses against those potentially better-informed agents.

Motivated by these considerations, this paper explores the short- and long-term impact of official spot interventions on different dimensions of the intraday process of price formation in the forex market: quotes, quotes' revisions, absolute and proportional bid-ask spreads, price volatility, and investors' order flow. ${ }^{4}$ To that end, we develop a model of sequential exchange rate trading in the spirit of Garman (1976), Brock and Kleidon (1992), and Saar (2000a, b). There are two assets in the economy, a domestic and a foreign currency-denominated riskless bond. Investors reach the market one at a time, according to an exogenously selected arrival process, and trade just once. The investors' population is made of two different types of pricetaking agents, depending on their initial endowment of the domestic asset (high or low). These agents formulate orders that maximize constant absolute risk-aversion (CARA) expected utility of their final wealth. Liquidity is provided, and prices are set by a monopolist or by competitive risk-neutral market-makers who maximize expected instantaneous profits from trading under a market-clearing constraint and while facing uncertainty about the identity of the next incoming order.

We then extend this basic setup by including a stylized monetary authority whose actions are fully consistent with the available literature on official intervention. At the core of our work is in fact Madhavan's (2000) recognition of "the potential value from combining microstructure and macro variables within a single model" to enhance the understanding of the global currency markets. We concentrate on the most common form of intervention, i.e., on CB transactions that, by leaving the domestic monetary base unchanged, do not affect monetary policy and economic fundamentals. The effectiveness of sterilized interventions, as those actions are known in the literature, is still controversial and, as such, at the center of the current theoretical and empirical debate. The resulting model, albeit parsimonious, allows us to consider the two major channels of effectiveness of sterilized intervention, imperfect substitutability (or portfolio balance) and signaling, without sacrificing its analytical tractability. ${ }^{5}$

Our framework integrates a rational, but not necessarily profit-maximizing CB in a purely microstructure setting in which orders do not clear simultaneously, dealers hold different degrees

\footnotetext{
${ }^{3}$ Bank Negara is commonly believed to be responsible for a series of speculative attacks against many currencies from 1990 to 1992. However, according to Brown (2000), Bank Negara was "using its inside information as a member of the club of central bankers to speculate in currencies, sometimes to an amount in excess of $\$ 1$ Billion a day," since late 1989. At that time, Dr. Mahathir, Malaysia's Prime Minister, was reported claiming that the CB's practices were no more than "active reserve management."

${ }^{4}$ Lyons (2001) defines the spot market "the essence of the forex market." However, CBs can (and sometimes do) use alternative financial instruments for their interventions, like interest rates, forward contracts, or derivatives. There is some agreement (e.g., Eaton and Turnovsky (1984), Miller (1998), Sarno and Taylor (2001)) that spot trades are nontheless the most effective transactions in signaling CB's intentions and objectives to the market at large, as they have a direct cost for the CB.

${ }^{5}$ In the first case (e.g., Branson (1983, 1984)), CB trades alter the composition of the agents' portfolios. The exchange rate must then shift to change the expected returns from holding less than perfectly substitutable domestic and foreign bonds, and so induce the market to adjust to the new currency supply. In the second case (Mussa (1981), Bhattacharya and Weller (1997)), intervention affects exchange rates by providing investors and forex dealers with supposedly new and relevant information.
} 
of market power, and there is full information or different degrees of asymmetric information among the players. Our analysis of the role of a monetary authority, and of its effectiveness in managing the exchange rate in such a setting is, to our knowledge, an original contribution to the economic and financial literature. Our model generates both macro and microstructure implications. We show that endogenously formulated sterilized CB intervention, or merely its likelihood, has long-lived effects on the exchange rate when the actions of the monetary authority are informative about policy objectives or economic fundamentals, or when the threat of future intervention is significant and credible, for these circumstances lead uninformed investors or dealers to a permanent revision in their beliefs. Portfolio balance effects of CB trades are instead short-lived because of trading occurring sequentially, and not because of high asset substitutability, as often maintained in the literature.

We also find that such interventions are more successful when dealers compete with each other for the incoming trade. Intuitively, competition induces the dealers to transfer any additional revenue or loss they expect from trading with the $\mathrm{CB}$ onto the population of investors. This is accomplished by greater and generally asymmetric revisions of their bid and ask quotes. The impact of currency management policies on intraday quotes and absolute and proportional spreads is not unidirectional, and depends crucially not only on the degree of market power held by the forex dealers, as suggested above, but also on the sign and magnitude of the announced and realized intervention, on the perceived likelihood of a future intervention to occur, and on the transparency of the order flow induced by the intervention.

Closely related to our study are two recent papers, Naranjo and Nimalendran (2000) and Evans and Lyons (2001). Naranjo and Nimalendran explain the estimated increase in daily bidask spreads in proximity of unexpected interventions in the Deutschemark/U.S. Dollar market with adverse selection considerations. Their argument however depends crucially on the restrictive assumptions that $\mathrm{CBs}$, acting as large insiders, attempt to disguise their presence among uninformed investors and that their trades are exogenously effective. Evans and Lyons (2001) instead concentrate exclusively on the portfolio balance impact of order flow uncertainty induced by exogenous interventions on inter-dealer trading by extending the "hot potato" model of Lyons (1997). Information asymmetry and signaling effects, although of greater empirical significance (e.g., Edison (1993), Pasquariello (2002)), are therefore ruled out by construction. Furthermore, their model is in the spirit of simultaneous-move games, and does not study the intraday dynamics of bid-offer spreads.

The paper is organized as follows. Section 2 describes the basic model. In Section 3 we derive our first set of results for quotes and spreads assuming full information and for different degrees of market power held by the dealers. Section 4 extends the setting of the previous two sections to a unifying example of information asymmetry between the $\mathrm{CB}$ and the population of investors and dealers. Section 5 explores the effects of such additional uncertainty on the equilibrium process of price formation using sequential stages of a stylized trading day in the forex market. In Section 6 we conclude by summarizing our results and outlining potential extensions of our work. 


\section{The basic model}

Foreign exchange (forex) markets share with most financial markets the attribute that investors do not necessarily hit dealers's quotes with orders at the same time. Uncertainty regarding the nature and the timing of the incoming order affects the way dealers formulate prices at which they are willing to sell and to buy the traded asset, in this case the currency. Hence, they use the arrival of orders, and their characteristics (like their size and sign) to extract information not only regarding the exchange rate but also regarding the composition of the investors' population at large or the nature of investors' demand function.

The financial literature has developed several models for the sequential arrival of investors to a market. ${ }^{6}$ The basic setup of our model is similar in spirit to Bhattacharya and Weller (1997) and Saar (2000a, b). In the following subsections we describe such framework, define how the three categories of players we consider in this paper, investors, dealers (MM), and Central Banks (CB), interact in the economy, and present a base scenario for equilibrium quotes and spreads under two extreme degrees of market power (monopoly and perfect competition) and no CB intervention. Finally, we allow the CB to be part of the general population of investors, and derive our first set of results in the context of full information. We will introduce information asymmetry in Section 4.

\subsection{Assets}

There are two assets in the economy, a riskless bond paying $R>1$ units of the domestic currency (e.g., U.S. dollars, USD) and a riskless bond paying $R_{F}>1$ units of the foreign currency (e.g., British pounds, GBP) at time $T^{\prime}$. We assume, for sake of simplicity, that investors, dealers, and CBs are dollar-based agents, i.e., that the domestic currency is the numeraire. Hence, the dollar payoff at time $T^{\prime}$ of the GBP-denominated bond, $F$, is uncertain (and consequently risky) because the amount of USD necessary to buy one GBP at time $T^{\prime}, S_{T^{\prime}}$, is uncertain, i.e., $F=R_{F} S_{T^{\prime}}$. In this economy, trading exchange rates is then equivalent to trading USD versus GBP-denominated T-bills. To ease the notation, we let $R_{F}$ be equal to 1 , so to interpret $F$ simply as the uncertain future value of the exchange rate between USD and GBP. We assume that nature draws $F$ at time $T^{\prime}$ from a Normal distribution with (random and chosen at time zero) mean $f$ and variance $\sigma_{F}^{2}$. Only in a full information scenario all players (investors, dealers, and monetary authority) are aware of nature's initial selection for $f$. The exchange rate market opens at time zero, and trading occurs until time $T<T^{\prime}$. The interval $[0, T]$ can be interpreted as a short period of time, for example one trading day. The distance between $T$ and $T^{\prime}$ is controlled by $R$; for a given set of annual riskless rates, higher $R$ (and $R_{F}$ ) implies that the long-term realization $S_{T^{\prime}}$ is further in the future. Intraday interest rates are assumed to be zero.

Arrivals of orders, like in traditional sequential trading models, are here exogenous and driven by an orderly point process $G(t)$ (as in Saar $(2000 \mathrm{a}, \mathrm{b})$ ), so that only one arrival is allowed at any discrete point in time $t$. We assume that for each $t \in[0, T]$ there is a probability $l \in[0,1]$ of a CB's order, and a probability $(1-l)$ of an investor's order arriving to the market. The

\footnotetext{
${ }^{6}$ Models of non-simultaneous trading have been used to study the problem of a dealer facing incoming orders that move him away from his desired inventory position (as in Amihud and Mendelson (1980) and Ho and Stoll (1981)), or of a market-maker facing incoming orders from potentially better-informed traders (e.g., Glosten and Milgrom (1985) or Easley and O'Hara (1987, 1991, and 1992)).
} 
parameter $l$ can alternatively be interpreted as the frequency (or credibility) of CB's arrivals during a trading day, and it is assumed to be exogenously chosen by the Central Banker, i.e., by the Governor, policymakers, politicians, etc.

\subsection{Investors}

Investors are risk-averse, and may belong to two different types, 1 and 2, depending on their initial endowment of risky GBP-denominated T-bills and riskless USD-denominated T-bills, $\bar{X}_{1}$ and $\bar{X}_{2}$, and $\bar{B}_{1}$ and $\bar{B}_{2}$, respectively. There is a fraction $q$ of investors of type 1 in the general population of investors; hence $q$ (always known to all agents) can be interpreted as the probability that, if an investor's order arrived to the market, that order comes from a type 1 investor. We assume that $\bar{X}_{1}>\bar{X}_{2}$, i.e., that type 1 investors are potentially net sellers, and type 2 investors are potentially net buyers of GBP. While this assumption is not relevant to any of the results we obtain in the paper, it nonetheless facilitates the interpretation of investors' actions and the analysis of the demand elasticity of the investors' population. All investors maximize the expected CARA utility of their final wealth, $W_{T^{\prime}}$, with the same degree of risk-aversion $\alpha$. All investors take market prices as given, and do not return to the market after they traded to rebalance their endowment portfolio. ${ }^{7}$ In such setting, the optimal demand schedule for GBP and USD-denominated T-bills, $Q_{i, t}$ and $B_{i, t}$, respectively, of an investor belonging to type $i$ $\in(1,2)$, with information set $I_{i, t}$, are the solution of the following problem:

$$
\begin{aligned}
\max _{Q_{\mathrm{i}, \mathrm{t}}, B_{\mathrm{i}, \mathrm{t}}} E\left[-e^{-\alpha W_{\mathrm{i}, \mathrm{T}^{\prime}}} \mid I_{i, t}\right] & \\
\text { s.t. } \quad B_{i, t}+S_{t} Q_{i, t} & =\bar{B}_{i}+S_{t} \bar{X}_{i} \\
R B_{i, t}+F Q_{i, t} & =W_{i, T^{\prime}},
\end{aligned}
$$

where the USD (GBP) price of the USD (GBP)-denominated riskless bond is set to unity. The solution of this problem is well-known in the financial literature, and the optimal demand for an investor of type $i$ is given by $^{8}$

$$
Q_{i, t}=\frac{E\left[f \mid I_{t}\right]-R S_{t}}{\alpha \sigma_{F}^{2}}
$$

Under full information $E\left[f \mid I_{t}\right]$ is equal to $f$, and we can write the expression for the net demand for GBP-denominated T-bills of type $i$ investors as

$$
X_{i, t}=\frac{1}{\pi}\left(\frac{f}{R}-S_{t}\right)-\bar{X}_{i}
$$

where $\pi=\frac{\alpha \sigma_{F}^{2}}{R}$ is a parameter controlling the elasticity of investors' demand for risky assets, i.e., the less than perfect substitutability of domestic and foreign currency-denominated T-bills. A positive (negative) $X_{i, t}$ is interpreted as a buy (sell) order for the foreign currency.

\footnotetext{
${ }^{7}$ This assumption is reasonable if we interpret the interval $[0, T]$ as one trading day.

${ }^{8}$ See Grossman and Stiglitz (1980). Bhattacharya and Weller (1997) use a similar description of investors' activity in the currency markets, and label those agents foreign exchange speculators.
} 


\subsection{Dealers}

As observed by Lyons (1995), most currency dealers tend to close their working day with a zero net position in their inventory. Why does this occur? Lyons suggests that, because trading in currencies might continue during the evening, the prospect of carrying an open position through the night without being able to properly monitor it is not particularly appealing to both the dealers and the banks for which they operate. There is also some anecdotal evidence of the fact that most forex dealers, because of the pace of the trading day, do not formulate any opinion about where the true value of the currency should be, hence they do not trade as investors.

It then seems reasonable to imagine that forex dealers, in setting their quotes, would try to impose no expected (positive or negative) drift to their inventory at any point in time, but simply to profit from incoming orders by charging a bid-ask spread as compensation for providing liquidity services to investors (and $\mathrm{CBs}$ ). It is equally reasonable to suspect that different degrees of market power held by currency dealers have an effect on the process of intraday price formation, and on their ability to extract rents from the exogenously driven order flow. For some exchange rates, e.g., Yen versus USD (JPYUSD) or Euro versus USD (EURUSD), competition among dealers is intense and may exert a downward pressure on their compensation for the liquidity they provide to the market. However, for some other exchange rates, especially in emerging economies, there are often very few dealers providing that service on a regular basis and for significant trade sizes.

Consistently with this view of the activity of market-makers (MMs) in the forex markets, we consider the extreme cases of a monopolist dealer, and of a set of competitive dealers. In the monopolist case, at each $t$ the dealer sets quotes that maximize the expected profit per unit time, i.e., that maximize the expected cash inflows from selling shares minus the expected cash outflows from buying shares to and from investors (and maybe the CB). ${ }^{9}$ Many competing dealers instead set the same quotes such that the expected profit for the representative MM is equal to zero, by virtue of Bertrand competition. ${ }^{10}$ In order to isolate the impact of interventions on quotes and spreads, we assume that the MMs do not face any order processing costs or inventory control, i.e., that our stylized currency market is frictionless. Then, if we define $M_{t}$ as the information set available to the dealer(s) at time $t$, before the next incoming order arrives, $S_{i, t}$ as the price quoted to an investor of type $i$, and $S_{t}^{C B}$ as the price quoted to the $\mathrm{CB}$, the expected instantaneous profit $\Pi_{t}$ earned by the MM can be represented as

$$
E\left[\Pi_{t} \mid M_{t}\right]=(1-l) q X_{1, t} S_{1, t}+(1-l)(1-q) X_{2, t} S_{2, t}+l X_{t}^{C B} S_{t}^{C B},
$$

where $X_{t}^{C B}$ is the $\mathrm{CB}$ order. In the full information scenario, $M_{t}$ includes all parameters controlling the terminal payoff of the risky GBP bond, all characteristics of the investor (and CB) population, including their demand functions, and all past transactions.

In both cases, dealers are subject to the constraint that their inventory has no expected drift at each point in time. Hence, before an order actually arrives, quotes are set so that the market is always cleared by balancing the expected flow of currency bought and sold at every instant $t$.

\footnotetext{
${ }^{9}$ Amihud and Mendelson (1980) use a similar specification, but allow the MM to set prices so that his expected inventory position may lay inside some pre-specified bounds.

${ }^{10}$ This is also the case in many other sequential trading models (e.g., Glosten and Milgrom (1985) and Madhavan (1992), just to name a few) under the assumptions of risk-neutrality and no capital constraint for the MM. For more on this topic see Saar (2000a).
} 
The no-expected inventory condition at each point in time is

$$
E\left[Z_{t} \mid M_{t}\right]=(1-l) q X_{1, t}+(1-l)(1-q) X_{2, t}+l X_{t}^{C B}=0,
$$

where $\left(-Z_{t}\right)$ is the instantaneous inventory position of the dealer(s). This assumption captures the nature of MMs' activity in the currency markets. It in fact translates our earlier observation that forex dealers basically attempt to balance the expected flow of currency bought and sold at each moment without imposing any expected drift to their inventory, instead of behaving similarly to investors holding GBP-denominated bonds until maturity.

At each instant $t$ dealers in this economy set potential (or reservation) prices, i.e., quotes at which they are willing to buy, if a type 2 investor arrives, to sell, if a type 1 investor arrives, and to trade with a $\mathrm{CB}$, if the monetary authority intervenes. When an investor's order hits the dealers' screens, the only equilibrium price is going to be the one, and the one only, resulting from the most recent transaction. We define the expected transaction price as $S_{t}^{*}=q S_{1, t}+(1-q) S_{2, t}$. Nonetheless, it is that declaration of intents by the dealer(s) that constitutes the bid and offer quotes. Trading is not anonymous with respect to whether the incoming order is from the population of investors or from the $\mathrm{CB}$, as it appears to be the case in most forex markets. However, MMs can distinguish the type of an arriving investor just from the sign and size of the submitted order.

We start with the assumption that $l$ is zero, i.e., that the "true" likelihood of the CB to intervene during the interval $[0, T]$ is zero and known to all market participants (as is $f$ ), and then solve the problem of both the monopolist and the competitive MMs. To this end, we adopt the convention that each incoming order is split evenly among all dealers quoting the same price, as in Saar (2000a). We report the resulting equilibrium prices in Proposition 1, as they serve as a benchmark scenario to evaluate the impact of the introduction of a CB in the next subsection.

Proposition 1 With full information and $l=0$, the competitive $M M s^{\prime}$ reservation bid and ask quotes at any point in time $t$, before an investor's order arrives, are

$$
S_{1, t}^{l=0}=S_{2, t}^{l=0}={ }^{C} S_{t}^{*, l=0}=\frac{f}{R}-\pi X^{*},
$$

where $X^{*}=q \bar{X}_{1}+(1-q) \bar{X}_{2}$. The resulting bid-ask spread is equal to zero. The monopolist MM's reservation bid and ask quotes are instead given by

$$
\begin{aligned}
& S_{1, t}^{l=0}=\frac{f}{R}-\pi X^{*}-\frac{\pi(1-q)}{2}\left(\bar{X}_{1}-\bar{X}_{2}\right), \\
& S_{2, t}^{l=0}=\frac{f}{R}-\pi X^{*}+\frac{\pi q}{2}\left(\bar{X}_{1}-\bar{X}_{2}\right) .
\end{aligned}
$$

The expected transaction price and the absolute spread are then, respectively,

$$
{ }^{M} S_{t}^{*, l=0}=\frac{f}{R}-\pi X^{*}={ }^{C} S_{t}^{*, l=0}=S_{t}^{*, l=0}
$$

and

$$
S_{2, t}^{l=0}-S_{1, t}^{l=0}=\frac{\pi}{2}\left(\bar{X}_{1}-\bar{X}_{2}\right) .
$$


Proof. See Section 1.2 in Saar (2000a).

In this study we define the bid-ask spread with respect to the investors as the difference between the prices dealers quote to type 2 and type 1 traders, i.e., $S_{2, t}-S_{1, t}$. In the remainder of the paper, we will specify conditions on the model's parameters such that type 1 investors are always net sellers hitting the dealers' bid quotes $S_{1, t}$ and type 2 investors are always net buyers hitting the dealers' ask quotes $S_{2, t}$. For example, in Proposition 1 we have that $X_{2, t}>0$ and $X_{1, t}<0$ in both the monopolistic and competitive scenarios if and only if $\bar{X}_{1}>\bar{X}_{2}$, as we previously assumed. Under these conditions, we can interpret our spread as a wedge between the price at which the dealers are willing to sell GBP to type 2 investors and the price at which the dealers are willing to buy GBP from type 1 investors.

The expected transaction price from the competitive dealers' setting, ${ }^{C} S_{t}^{*, l=0}$ in Eq. (6), corresponds to the price we would observe if all investors arrive at the same time in a competitive market, i.e., to the market-clearing price. Consequently, the no-expected drift condition of Eq. (5) in the full information scenario is equivalent to the market-clearing condition in a competitive equilibrium. The price of the GBP-denominated bond is equal to the discounted future payoff (the risk-neutral component of Kyle (1985)) minus a risk adjustment factor that is necessary to induce risk-averse USD-based investors to hold assets in GBP. This component depends positively on the expected amount of foreign currency already held by the investors $X^{*}$ (hence on the relative types' weight $q$ ) and on the parameter measuring the elasticity of the investor's demand $\pi$. No spread emerges in this case, because of the assumption of full information in this otherwise frictionless forex market.

A monopolist dealer uses his market power to extract positive rents from investors by charging a higher ask to the net buyer type $(i=2)$, and by paying a lower bid to the net seller type $(i=1)$. The ensuing spread depends on the parameters controlling investors' order flow in $\pi$, and is equal to half of the unconstrained profit-maximizing wedge $\pi\left(\bar{X}_{1}-\bar{X}_{2}\right)$ because the market-clearing condition is binding. The expected transaction price in Eq. (9), ${ }^{M} S_{t}^{*, l=0}$, is nevertheless the same as in the competitive scenario. As evident from Eqs. (7) to (10), this result does not depend on whether type 1 investors hold more or less of the GBP bond than type 2 investors, but on whether there is trading in this economy, i.e., $\bar{X}_{1} \neq \bar{X}_{2}{ }^{11}$

\subsection{Central Bank}

Most Central Banks frequently intervene in the foreign exchange market in order to manage otherwise free-floating rates, to comply with existing international currency agreements, to serve broad macroeconomic and monetary agendas, or as a result of domestic political pressure. In less frequent occasions, interventions are conducted with the purpose of remedying balance of payments problems, although such actions are explicitly prohibited by the IMF Article 4, Section 1. In some circumstances CBs have also acted in pursuit of purely speculative motives, as in the case of Bank Negara in the early 1990s, although uncovering their profits and losses from

\footnotetext{
${ }^{11}$ It is easy to show that, by plugging the equilibrium monopoly or competitive quotes in the optimal demand function of type 1 and type 2 investors of Eq. (3), both $X_{1, t}$ and $X_{2, t}$ are equal to zero for $\bar{X}_{1}=\bar{X}_{2}$. Therefore, when otherwise identical investors have the same initial endowments, no risk-sharing (hence, no trading) occurs in the market.
} 
foreign exchange trading may be a daunting task. ${ }^{12}$ Lewis (1995) identifies several common features in the interventions conducted by the Federal Reserve (Fed), Bank of Japan (BoJ), and Bundesbank (BuBa) on DEMUSD and JPYUSD between 1985 and 1987. First, most of those actions were aimed at preventing the above exchange rates from moving away from some target levels. Second, in few but significant cases the interventions went in the opposite direction with respect to the exchange rate. It is possible that, in these circumstances, the CBs were attempting either to stabilize fluctuations of their domestic currencies around the target levels, or to speculate against them by following the trend and, in doing so, preserving their expected future wealth from depletion. Third, although the intervention events may have been announced to or observed by the market, the magnitude of the incoming CB orders was usually not, as also suggested by Goodhart and Hesse (1991), among others.

Policy and wealth-preservation (or speculative) motives may be conflicting. Suppose in fact that a CB believes, based on its superior knowledge of the domestic economy, that the domestic currency (USD) is fundamentally overvalued, and that it is reasonable to expect its reversion, i.e., that $S_{t}<f$. A sudden devaluation however could create excessive inflationary pressures. To attenuate those pressures, the CB could set an intermediate target level for the exchange rate, $\bar{S}$, between $S_{t}$ and $f$, and sell some amounts of foreign currency (GBP) to prevent the exchange rate from breaking its current trend too rapidly toward its long-term value. Nonetheless, given its knowledge of $f$, the CB's action, if effective on $S_{t}$, is not properly profit-maximizing (as buying GBP would be instead) and leads to a reduction of its future expected wealth.

In this paper, we model such trade-off between management of the exchange rate around a specified target and wealth-preservation (or speculative) motives in a parsimonious way by assuming that the $\mathrm{CB}$ chooses the net amount of foreign (domestic) currency to buy/sell, $X_{t}^{C B}$ $\left(B_{t}^{C B}\right)$, that minimizes the following loss function:

$$
L(\bar{S}, \lambda)=\left[E\left(S_{t}^{*} \mid \mathbf{z}_{t}\right)-\bar{S}\right]^{2}-\lambda E\left(W_{T^{\prime}}^{C B} \mid \mathbf{z}_{t}\right),
$$

in which $W_{T^{\prime}}^{C B}=R\left(B_{t}^{C B}+\bar{B}_{t}\right)+F\left(X_{t}^{C B}+\overline{R E S}_{t}\right)$, subject to the budget constraint

$$
B_{t}^{C B}=-S_{t}^{C B} X_{t}^{C B}
$$

where $\mathbf{z}_{t}$ is the CB's information set at time $t, E\left(S_{t}^{*} \mid \mathbf{z}_{t}\right)=q S_{1, t}+(1-q) S_{2, t}$ is the expected transaction price from trading between the dealer(s) and the population of investors, $\tau \in(0, t)$ is when the most recent past intervention occurred, and $\overline{R E S}_{t}=\overline{R E S}_{\tau}+X_{\tau}^{C B}$ and $\bar{B}_{t}=\bar{B}_{\tau}+B_{\tau}^{C B}$ are the endowments of foreign and domestic currency-denominated bonds held by the $\mathrm{CB}$ at time $t$ before intervening. ${ }^{13}$

Let's examine carefully the loss function of the CB. The first term measures the quadratic distance between $E\left(S_{t}^{*} \mid \mathbf{z}_{t}\right)$ and an exogenously selected target level for the exchange rate. $E\left(S_{t}^{*} \mid \mathbf{z}_{t}\right)$ is a relevant price, as it represents the expected market-clearing exchange rate that would be observed if all investors and dealers could trade simultaneously at time $t$. It is beyond the scope

\footnotetext{
${ }^{12}$ Taylor (1995) offers an overview on the economics of official interventions. See also Brown (2000) and Chancellor (2000) for further details on the actions by the Malaysian Central Bank in the 1990s.

${ }^{13}$ Given that the interval $[0, T]$ represents a short period of time (e.g., one trading day), we abstract from the issue of a monetary authority being unable to intervene because of lack of reserves by assuming that the initial endowments $\overline{R E S}_{0}$ and $\bar{B}_{0}$ are big enough so that $\overline{R E S}_{t}>0$ and $\bar{B}_{t}>0$ before and after an intervention occurred.
} 
of this paper to analyze how a target level for the exchange rate is selected and agreed upon by domestic and foreign monetary authorities; it suffices for us to know that the chosen level $\bar{S}$ is assumed to guide the CB's action in the forex market. ${ }^{14}$ At each point in time $t$ the CB is myopic, i.e., like the investors, does not expect to return to the market by the end of the trading period $T$. This assumption, by implicitly imposing that the CB's trading activity is controlled exogenously by the parameter $l$, allows us to abstract from the complex macro issue of analyzing its endogenous intertemporal strategic behavior (explored, for example, by Cadenillas and Zapatero $(1999,2000)$ ) and to make the problem of the monetary authority more tractable, although at the cost of less realism. We nonetheless decided to bear this cost, given our micro objective to study the impact of the existence of a (not necessarily profit-maximizing $\mathrm{CB}$ ) on the relationship between dealers and investors over a single trading day $[0, T]$.

The second component of $L(\bar{S}, \lambda)$ incorporates what we called the wealth-preservation (or, alternatively, speculative) motive in the decision process of the CB. Intervention has a cost, and this cost is obviously higher when CB's actions are unprofitable from a pure trading perspective. We assume that actions reducing its expected future wealth are linearly painful for a risk-neutral, price-taking $\mathrm{CB}$, and use the parameter $\lambda$ to control for its potential trade-off between policy motives and speculation in selecting the optimal size (and sign) of $X_{t}^{C B} .{ }^{15}$ The specification of Eq. (11), with a CB not directly motivated by profit considerations, is similar in spirit to Stein (1989) and to the objective functions adopted by Bhattacharya and Weller (1997) and Vitale (1999). The budget constraint in Eq. (12) implies that each GBP trade by the CB is accompanied by an open-market trade in the opposite direction. In addition, as mentioned in Section 2.1, both the fundamental value of the currency $(f)$ and the realized long-term exchange rate $\left(S_{T^{\prime}}\right)$ are drawn by nature independently of any $\mathrm{CB}$ action. From these assumptions it follows that, in our setting, interventions are always sterilized.

Nonetheless, our model does not exogenously impose a relationship between the actions of the stylized CB and the exchange rate, as, for example, in Naranjo and Nimalendran (2000). What is then the market friction that allows interventions to be potentially effective in this economy? The CB uses the market-clearing condition (Eq. (5)) and the investors' optimal net demand for GBP-denominated bonds (Eq. (3)) to conjecture that the expected transaction price $q S_{1, t}+(1-q) S_{2, t}$ is given by

$$
E\left(S_{t}^{*} \mid \mathbf{z}_{t}\right)=\frac{E\left[f \mid I_{t}\right]}{R}-\pi X^{*}+\pi L X_{t}^{C B},
$$

where $L=\frac{l}{1-l} \cdot{ }^{16}$ Hence, for a given $l>0$, the optimal intervention schedule $X_{t}^{C B}$ for each price level $S_{t}^{C B}$ minimizes the loss function of Eq. (11) subject to Eq. (13) and the wealth constraint of Eq. (12). The following result ensues.

\footnotetext{
${ }^{14}$ The CB's loss function can be easily generalized to the case of a target band of fluctuation $\left(\bar{S}_{L}, \bar{S}_{H}\right)$ for the currency by specifying the policy term in Eq. (11) as $\left[E\left(S_{t}^{*} \mid \mathbf{z}_{t}\right)-\frac{1}{2}\left(\bar{S}_{L}+\bar{S}_{H}\right)\right]^{2}$, i.e., in terms of movements of $S_{t}^{*}$ away from the center of the band.

${ }^{15}$ Observe that Eq. (11) can alternatively be interpreted as the Lagrangian for the minimization of its policy component under a wealth constraint. Neely (2000) finds a small but significant correlation between CB intervention and changes in its reserves of foreign assets.

${ }^{16}$ Eq. (13) follows straightforwardly from the market-clearing condition $E\left[Z_{t} \mid M_{t}\right]=0$ when the optimal demand for GBP of type $i, X_{i, t}$, is replaced by the expression in Eq. (3) and the definition for $E\left(S_{t}^{*} \mid \mathbf{z}_{t}\right), q S_{1, t}+(1-q) S_{2, t}$ (as $\mathbf{Z}_{t}$ includes $q$ ) is applied.
} 
Proposition 2 With full information and $l>0$, the demand function of the $C B$ is given by

$$
X_{t}^{C B}=\gamma\left[\bar{S}-\left(\frac{f}{R}-\pi X^{*}\right)+\frac{\lambda}{2 \pi L}\left(f-R S_{t}^{C B}\right)\right],
$$

where $\gamma=\frac{1}{\pi L}$.

Proof. Straightforward from the first order condition of the constrained minimization of the loss function $L(\bar{S}, \lambda)$ described in Eq.(11) with respect to $X_{t}^{C B}$. The second order condition, $2 \pi^{2} L^{2}>0$, is always satisfied.

It is evident from Eq. (13) that, ceteris paribus for $S_{t}^{C B}$, the optimal intervention size declines for higher $l$. This means that the less likely is the $\mathrm{CB}$ to intervene (or the less frequently the CB intervenes), the higher is, for a given sign, CB's order size. Intuitively, a lower $l$ makes the threat of intervention less significant for the dealers, therefore a bigger $X_{t}^{C B}$ is necessary to move the expected transaction price toward the target rate $\bar{S}$. This property of Eq. (13) is consistent with some evidence in Naranjo and Nimalendran (2000); indeed, it is there shown that BuBa's interventions in the DEMUSD market are more frequent than the Fed's, but smaller in absolute dollar size.

The CB's optimal demand function depends, in a very intuitive fashion, on both the policy and wealth-preservation motives. The CB needs to buy (sell) the foreign currency to push the expected transaction rate closer to the target level, if the difference between $\bar{S}$ and the price that would emerge in a purely competitive economy with no monetary authority $(l=0), \frac{f}{R}-\pi X^{*}$, is positive (negative). If $\bar{S}>\frac{f}{R}-\pi X^{*}$, the CB is chasing the trend, i.e., is attempting to induce a faster depreciation of $S_{t}^{*}$ toward its long-term fundamental value, hence it buys GBP. ${ }^{17}$ If instead $\bar{S}<\frac{f}{R}-\pi X^{*}$, the $\mathrm{CB}$ is leaning against the wind, i.e., is attempting to resist $S_{t}^{*}$ 's long-term depreciation trend by selling GBP. ${ }^{18}$ The second term in $X_{t}^{C B}$ is more properly wealth-preserving. It in fact implies that the CB buys more GBP-denominated bonds (and simultaneously sells more dollar-denominated T-bills) if the expected net future value (NFV) in dollars of that investment, $f-R S_{t}^{C B}$, is higher. The amounts of GBP bought (or sold) by the GBP depend crucially on $\lambda$, the parameter controlling the trade-off between policy and speculative motives. This trade-off is not surprisingly highest when the monetary authority is trying to lean against the wind and, in doing so, is reducing its expected future wealth.

The likelihood of an order to arrive from the monetary authority $(l)$ and the elasticity of investors' demand for GBP $(\pi)$ affect (via $\gamma$ ) the impact of both motives on the size of the CB orders, consistently with the intuition of the portfolio balance theories of $\mathrm{CB}$ intervention. If $l$ goes up, the CB needs to trade a smaller amount of currency to achieve its objectives, since its trade has a bigger impact on the expected transaction price $S_{t}^{*}$, as evident from Eq. (13).

${ }^{17}$ In particular, if $\bar{S}>\frac{f}{R}$ we say that the CB is riding the wave, i.e., is aggressively pursuing a depreciation of the dollar beyond the long-term risk-neutral rate $\frac{f}{R}$. We already observed that such competitive devaluations are explicitly forbidden by the IMF Article 4, Section 1.

${ }^{18}$ In Section 2.1 we assumed that $R>1$ and $R_{F}=1$ (or, more generally, $\frac{R_{F}}{R}<1$ ). If we had assumed that $R<1$ (or $\frac{R_{F}}{R}>1$ ) insomuch that, when $l=0$, the dollar were weaker than its long-term expected value $\left(S_{t}^{*, l=0}>E\left[S_{T^{0}}\right]=f\right)$, then the $\mathrm{CB}$ would be chasing the trend if $\bar{S}<\frac{f}{R}-\pi X^{*}$, and leaning against the wind if $\bar{S}>\frac{f}{R}-\pi X^{*}$. For simplicity, we ignore this possibility and concentrate just on the scenario in which $R>1$. 
Moreover, ceteris paribus for $S_{t}^{C B}$, if investors are more risk-averse (higher $\alpha$ ), if there is more uncertainty surrounding the long-term exchange rate $F$ (higher $\sigma_{F}^{2}$ ), or if investors have a higher expected endowment of GBP (higher $X^{*}$ ), then their demand for GBP is less elastic. Hence, a bigger increase (decrease) in the potential prices is needed to induce them to hold more (less) GBP and push $S_{t}$ toward the high (low) $\bar{S}$. Therefore, a bigger positive (negative) intervention is necessary. It is also easy to verify that $X_{t}^{C B}$ depends positively on the selected target level, on the NFV of investing in GBP-denominated assets, and on the expected individual endowment level $X^{*}$, but negatively on the quoted rate $S_{t}^{C B}$.

\section{The full information case}

In this section, we allow the $\mathrm{CB}$ to intervene with its orders $X_{t}^{C B}$. We then solve for the resulting equilibrium quotes for monopoly and competition among currency dealers, and present our first set of results under the assumption of full information, i.e., that all agents know all parameters of the economy (in particular $l, \bar{S}$, and $f$ ), demand functions, and past order flow. Full information restricts the effectiveness of CB's actions to the portfolio balance channel where investors (if risk-averse, as in our model) have to be compensated for having to hold more (or less) of the foreign currency than they otherwise would if $l=0$.

We construct the equilibrium in three steps. We first assume that in equilibrium dealers can always distinguish whether the incoming order is from a CB or from the population of investors, that they can just conjecture the investor's type from the size and sign of his observed order, and that they use this knowledge to formulate their reservation prices for each potential arrival. ${ }^{19}$ We also assume that the CB conjectures an expression for the expected transaction price in its loss function $L(\bar{S}, \lambda)$. Second, we compute type 1 and type 2 agents' optimal demands for GBPdenominated bonds and the optimal intervention at the reservation prices $S_{1, t}, S_{2, t}$, and $S_{t}^{C B}$. Finally, we show that the resulting investors' orders, given those prices, are indeed different, and that $E\left[S_{t}^{*} \mid \mathbf{z}_{t}\right]$ is indeed equal to $q S_{1, t}+(1-q) S_{2, t}$, confirming the dealers' and CB's initial guesses, respectively, as in classic fixed-point problems.

The full information hypothesis also implies that all agents (including investors) not only know exactly $X_{t}^{C B}$ of Eq. (14) but also observe the size of the trade executed by the CB. Available empirical evidence (in particular Goodhart and Hesse (1991), Lewis (1995), and Peiers (1997)) seems to suggest otherwise. Nevertheless, we choose to use this simplified scenario to explain the basic intuition for how dealers adjust their quotes in response to the (likelihood of) arrival of a CB order, and to understand why the adjustment does (or does not) induce a change in the spreads with respect to the basic case of Eq. (6) to (10). In ,Section 4 we eventually introduce information asymmetry among agents regarding some of the model's parameters and more realistic assumptions for their information sets, and study the impact of interventions on the process of price formation when the signaling channel of effectiveness is active as well.

\footnotetext{
${ }^{19}$ This assumption is reasonable, because it captures two typical aspects of OTC currency markets: lack of anonymous trading and, consequently, price discrimination. For more on the trading relationship between the CB and MMs for the domestic currency, see Peiers (1997).
} 


\subsection{The monopolist dealer}

We showed in Proposition 1 that a monopolist dealer widens an otherwise zero spread (in absence of inventory control or information asymmetry), centered around the competitive equilibrium exchange rate $\frac{f}{R}-\pi X^{*}$, to induce positive expected instantaneous profit from trading. What happens if we now introduce the possibility for the CB to hit the dealer with an order? Is the dealer's incentive to extract rents from investors somehow limited or enhanced by the likelihood of CB intervention being positive? The problem of the monopolist dealer is now

$$
\begin{gathered}
\max _{S_{1, t}, S_{2, t}, S_{\mathrm{t}}^{C B}}(1-l) q X_{1, t} S_{1, t}+(1-l)(1-q) X_{2, t} S_{2, t}+l X_{t}^{C B} S_{t}^{C B} \\
\text { s.t. } \quad E\left[Z_{t} \mid M_{t}\right]=(1-l) q X_{1, t}+(1-l)(1-q) X_{2, t}+l X_{t}^{C B}=0 \\
X_{t}^{C B}=\gamma\left[\bar{S}-\left(\frac{f}{R}-\pi X^{*}\right)+\frac{\lambda}{2 \pi L}\left(f-R S_{t}^{C B}\right)\right] \\
X_{i, t}=\frac{1}{\pi}\left(\frac{f}{R}-S_{i, t}\right)-\bar{X}_{i} \quad i=1,2 .
\end{gathered}
$$

Equilibrium construction in this setting generates the following result.

Proposition 3 With full information and $l>0$, the monopolist MM's reservation bid and ask quotes at any point in time $t$, before an investor's order arrives, are

$$
\begin{aligned}
& S_{1, t}=S_{1, t}^{l=0}+\pi L X_{t}^{C B} \\
& S_{2, t}=S_{2, t}^{l=0}+\pi L X_{t}^{C B},
\end{aligned}
$$

while the reservation exchange rate if a $C B$ intervenes at $t$ is given by

$$
S_{t}^{C B}=\omega_{1} \frac{f}{r}+\omega_{2} \bar{S}+\omega_{3} \pi X^{*} .
$$

The resulting absolute bid-ask spread is unchanged with respect to the benchmark of Eq. (10). The proportional spread does instead change, and is now equal to

$$
P S_{t}=\frac{S_{2, t}-S_{1, t}}{{ }^{M} S_{t}^{*}}=\frac{\pi\left(\bar{X}_{1}-\bar{X}_{2}\right)}{2\left(S_{t}^{*, l=0}+\pi L X_{t}^{C B}\right)} .
$$

Proof. See the Appendix. The proportional spread is computed using the "true" expected transaction price ${ }^{M} S_{t}^{*}$, and not the conventional mid-quote, because the probability that an investor's order is of type $1(q)$ is public information.

In short, Proposition 3 says that in equilibrium the monopolist dealer revises upward (downward) his quotes, with respect to the benchmark case of Proposition $1(l=0)$, if there is a positive probability that the $\mathrm{CB}$ will intervene at time $t$ buying (selling) the foreign currency, i.e., if $X_{t}^{C B}>0\left(X_{t}^{C B}<0\right)$. The absolute spread is unchanged, while the proportional spread declines (increases) for an expected positive (negative) $\mathrm{CB}$ order. The quote revision is given by

$$
\Delta S_{i, t}=\Delta S_{t}^{*}=\pi L X_{t}^{C B}=\frac{\pi L}{2 \pi L+\lambda R}\left(\bar{S}-\frac{f}{R}\right)+\frac{\pi}{2} X^{*} .
$$


What is the intuition for this result? Let's assume that it becomes known to the dealer (or that the $\mathrm{CB}$, by assumption truthful, announces) that from time $t$ onward, with probability $l>0$, an order $X_{t}^{C B}>0$ might arrive. Given the original set of quotes, $S_{i, t}^{l=0}$, this potential buy order creates an imbalance in the expected instantaneous dealer's inventory by adding to the originally flat position a negative drift component. In order for Eq. (5) to be satisfied or, equivalently, in order to clear the market, the MM increases $S_{1, t}$, the bid price at which he is willing to buy from net sellers, to reduce the size of the expected incoming sales, and $S_{2, t}$, the ask price at which he is willing to sell to net buyers, to increase the size of the expected incoming purchases. Because at the revised quotes net buyers buy less and net sellers sell more, investors' net demand is now expected to be a sale. This allows the dealer to clear the market when the $\mathrm{CB}$ is expected to be a net buyer of GBP. The magnitude of $\Delta S_{i, t}$ depends positively on the relative likelihood of CB intervention $(L)$ and on the elasticity of investors' demand for GBP-denominated assets (via $\pi$ ), as in portfolio balance models. $\Delta S_{i, t}$ is also symmetric, i.e., identical for both bid and offer, so to leave the absolute spread unchanged.

The bid-ask spread remains positive and unchanged because the MM is still trying to maximize his expected net cash flow from trading. The expected transaction price ${ }^{M} S_{t}^{*}$ does however increase, and the proportional spread declines. Vice versa, if the CB order were of negative sign, quotes would be revised downward symmetrically, the expected transaction price would decrease, so the proportional spread increase. Thus, in our setting the announcement of a fully expected CB intervention does have an impact on quotes and $P S_{t}$, and the direction of such impact depends on the expected sign of the $\mathrm{CB}$ trade. There are two reasons why this happens in our economy. First, although the probability of intervention is declared to every player, at each point in time $t$ the dealer still faces uncertainty about which order (investor versus monetary authority) is going to arrive. This uncertainty is embedded in the quotes' revisions via $L$. Second, the market-clearing constraint is binding. If $X_{t}^{C B}>0\left(X_{t}^{C B}<0\right)$, the dealer is expecting extra cash inflows (outflows) from the $\mathrm{CB}$, i.e., is anticipating an increase (decrease) of his expected net revenues from trading at time $t, E\left[\Pi_{t} \mid M_{t}\right]$. If the market had not to be cleared, the monopolist currency dealer would set quotes allowing him to retain the extra gain (to pass the extra cost) from CB intervention from (to) the population of investors. But this is not the case here: because investors' demand for the GBP-denominated asset is less than perfectly elastic, the no-expected net inventory condition obliges the dealer to give up some of the extra cash inflows (not to pass all the extra cash outflows) to investors in order to have $E\left[Z_{t} \mid M_{t}\right]=0$. To do so, the dealer reduces (increases) the proportional spread.

As suggested by Eq. (20), CB intervention is effective in moving the expected transaction price by an amount $\Delta S_{i, t}$ toward $\bar{S}$. In Figure 1 we plot the behavior of ${ }^{M} S_{t}^{*}$ for different levels of $l$, for a parametrization such that the CB is chasing the trend $\left(\bar{S}>\frac{f}{R}-\pi X^{*}\right) \cdot{ }^{20}$ In this example, $\Delta S_{i, t}$ induces an undershooting (overshooting) of ${ }^{M} S_{t}^{*}$ with respect to the target level $\bar{S}$ if the likelihood of intervention is relatively high (low). This occurs because for high (low) values of $l$ the monetary authority is expected to hit the dealer with a smaller (bigger) order size in order to achieve its objectives. In fact, at higher (lower) frequency $L$ the MM has more (less) scope to exploit his market power versus the CB (i.e., to maximize his profits while still clearing

\footnotetext{
${ }^{20}$ In all the simulations that follow in this paper, model's parameters are always chosen to ensure that the resulting ratio between the expected absolute size of investors' and CB orders is as similar as possible to available empirical estimates (roughly 12\%).
} 
the market); consequently he charges a higher (lower) $S_{t}^{C B}$, thus making the intervention more (less) expensive. The ensuing smaller (bigger) NFV of buying GBP reduces (increases) CB's incentive to weaken the dollar, hence the endogenously determined magnitude of its intervention operation. This effect is reinforced by the inverse relation between $X_{t}^{C B}$ and $L$ (for a given $S_{t}^{C B}$ ) described in Section 3.4. Therefore, the needed adjustment in the bid and offer rates to clear the market is bigger (smaller). Indeed, it is easy to show that, for any $\bar{S}<\frac{f}{R}, \frac{\partial \Delta S_{t}^{*}}{\partial l}<0$.

Because ${ }^{M} S_{t}^{*}=S_{t}^{*, l=0}+\Delta S_{t}^{*}$, we can interpret the first derivative of $\Delta S_{t}^{*}$ with respect to any of the parameters controlling CB's motives as measures of the price impact of its actions in the market. In particular, from Eq. (20) we compute $\frac{\partial^{\mathrm{M}} S_{\mathrm{t}}^{*}}{\partial X_{\mathrm{t}}^{\mathrm{CB}}}=\pi L$; thus, the ratio $\frac{\alpha \sigma_{\mathrm{F}}^{2} L}{R}$ represents the inverse of the relative market depth of the market (as in Kyle (1985)), a measure of how much the expected exchange rate moves from the no-intervention price $\frac{f}{R}-\pi X^{*}$ if there is a positive likelihood that the monetary authority is willing to transact one unit of GBP-denominated bonds at time $t(l>0)$. Not surprisingly, the currency market is less deep (from the CB's viewpoint), the price impact is higher, and the intervention is more effective when the coefficient of riskaversion, $\alpha$, and the volatility of the long-term value of GBP, $\sigma_{F}^{2}$, are higher, when the return paid by the riskless domestic bond is lower (as the underlying depreciation trend for the domestic currency is weaker, hence easier to challenge), or when the likelihood of government action is higher. Indeed, in such circumstances the monopolist dealer needs a bigger quote revision to clear the market for the same expected incoming CB trade, for example when risk-averse investors face more uncertainty surrounding nature's choice for $f$, and therefore their demand elasticity is lower.

Eventually, when the intervention does happen, that is if the incoming order is the one from the CB, there is no additional impact on quotes and spreads. This occurs because in full information the market is strong-form efficient and CB actions do not affect investors and dealers' beliefs about the fundamental value of the currency. In other words, as soon as the intervention is announced (and $l$ becomes positive) the dealer immediately and fully discounts this news in prices and spreads. However, in our setting trading is sequential and the market does not clear just once. Hence, the expected transaction price returns immediately to the pre-intervention levels as soon as $l$ is again equal to zero, because then no new investor needs to be rewarded (penalized) for having to hold more GBP than they otherwise would in the absence of active currency management by the government. Therefore, the impact of CB intervention remains on quotes and transaction prices just as long as the (exogenous) threat of arrival of orders from the monetary authority is present.

\subsection{The competitive dealers}

In this subsection we consider the case of competitive forex dealers, i.e., of dealers with no market power. We have shown in Proposition 1 that in such a circumstance, and under the assumptions of no inventory control, order processing costs, or information asymmetry, no spread arises in the resulting stylized currency market when $l=0$. Does that conclusion still hold if we introduce the positive likelihood of CB intervention at each point in time $t$ ? Along the lines of Section 3 , the equilibrium set of rates $S_{1, t}, S_{2, t}$, and $S_{t}^{C B}$ is the one that, given investors' $\left(X_{i, t}\right)$ and CB's $\left(X_{t}^{C B}\right)$ optimal demand functions, satisfies both $E\left[\Pi_{t} \mid M_{t}\right]=0$ and $E\left[Z_{t} \mid M_{t}\right]=0$. Competition among dealers drives the instantaneous expected net revenues from trading to zero, while the 
market-clearing condition still applies. These two restrictions are not sufficient to identify three reservation prices, hence we express the bid and offer quotes as functions of a free variable, $S_{t}^{C B}$. The following proposition ensues.

Proposition 4 With full information and $l>0$, the competitive $M M s^{\prime}$ reservation bid and ask quotes at any point in time $t$, as a function of $S_{t}^{C B}$, before an investor's order arrives, are

$$
\begin{aligned}
& S_{1, t}=S_{1, t}^{l=0}+\pi L X_{t}^{C B}-\left[\frac{\pi}{2}(1-q)\left(\bar{X}_{1}-\bar{X}_{2}\right)-\frac{\pi}{2}(\Gamma)\right] \\
& S_{2, t}=S_{2, t}^{l=0}+\pi L X_{t}^{C B}+\left[\frac{\pi q}{2}\left(\bar{X}_{1}-\bar{X}_{2}\right)-\frac{\pi q}{2(1-q)}(\Gamma)\right],
\end{aligned}
$$

where $\Gamma>0$ is given in the Appendix. A spread between the offer and the bid quotes does exists and is given by

$$
S_{2, t}-S_{1, t}=\frac{\pi}{2}\left(\bar{X}_{1}-\bar{X}_{2}\right)-\frac{\pi}{2(1-q)}(\Gamma)
$$

The sign and size of the spread depend on the selected parametrization of the economy. There exists a price $S_{t}^{C B}=S_{t}^{C B}(*)$ such that, for $X_{t}^{C B} \neq 0$, the above spread is equal to zero:

$$
S_{t}^{C B}(*)=S_{1, t}=S_{2, t}=\left(\frac{2 \pi L}{2 \pi L+\lambda R}\right) \bar{S}+\left(\frac{\lambda}{2 \pi L+\lambda R}\right) f .
$$

Proof. See the Appendix.

Proposition 4 contains several implications of the introduction of a $\mathrm{CB}$ in the competitive forex dealership scenario. As in the monopoly case, the new equilibrium bid and ask quotes differ from the prices reported in Proposition 1. Let's examine them more carefully. The chosen $S_{1, t}$ and $S_{2, t}$ depend on three different components, one of which, $\frac{f}{R}-\pi X^{*}$, is the competitive price arising when $l=0$ and investors' orders are cleared all at once. As such, this is our benchmark exchange rate. The remaining two are revisions induced by the positive likelihood of the $\mathrm{CB}$ to arrive. One of them, $\pi L X_{t}^{C B}$, affects symmetrically both bid and offer quotes. This is the adjustment that is needed to clear the market when $l$ and $X_{t}^{C B}$ are different from zero, and is similar, albeit not in magnitude to what we observed in the monopolistic scenario. If, for example, the $\mathrm{CB}$ is known to plan a sale of foreign currency $\left(X_{t}^{C B}<0\right)$ to strengthen the dollar toward a given low $\bar{S}$, dealers decrease the exchange rate at which they are willing to trade in order to achieve two related objectives. First, at the new levels, type 2 (type 1) investors will demand (offer) bigger amounts of GBP-denominated bonds. Second, the resulting expected exchange rate ${ }^{C} S_{t}^{*}$ will be closer to the target $\bar{S}$, thus reducing $X_{t}^{C B}$. Indeed, $X_{t}^{C B}$ is endogenously determined in our model, hence is crucially affected by where prices are expected to move. In other words, if quotes are symmetrically revised downward, the lower ${ }^{C} S_{t}^{*}$ induces a smaller intervention, while investors will be buying more GBP than they would have if $l=0$, thus facilitating the dealers' efforts to have a driftless expected inventory.

The exogenous price impact of CB intervention on the expected exchange rate (defined in Section 3.1 as $\left.\frac{\partial S_{\mathrm{t}}^{*}}{\partial X_{\mathrm{t}}^{\mathrm{CB}}}\right)$ is unaffected by the dealers' degree of market power. In fact, it ensues from the equilibrium quotes in Proposition 4 that ${ }^{C} S_{t}^{*}=E\left[S_{t}^{*} \mid M_{t}\right]$ is given by $S_{t}^{*} l=0+\pi L X_{t}^{C B}$, and 
that consequently $\frac{\partial^{\mathrm{C}} S_{\mathrm{t}}^{*}}{\partial X_{\mathrm{t}}^{\mathrm{CB}}}=\pi L=\frac{\partial^{\mathrm{M}} S_{\mathrm{t}}^{*}}{\partial X_{\mathrm{t}}^{\mathrm{CB}}}$. This occurs because the market-clearing condition is binding for both the monopolist and the competitive MMs. However, the equilibrium optimal $X_{t}^{C B}$ arises endogenously from the model; thus, ${ }^{M} X_{t}^{C B}$ is generally different from ${ }^{C} X_{t}^{C B}$, and so is ${ }^{M} S_{t}^{*}$ from ${ }^{C} S_{t}^{*}$, as we discuss in greater detail in the next subsection. Most interestingly, $l>0$ induces a wedge between bid and offer quotes: indeed, the bid-ask spread (in Eq. (23)) is generally different from zero, unless either $S_{t}^{C B}=S_{t}^{C B}(*)$ or $l=0$. To interpret this result, remember that our assumption of otherwise frictionless trading implies a benchmark zero spread when $l=0$ (Proposition 1). Therefore, by relaxing that assumption, we could more properly consider Eq. (23) as the change of an otherwise positive spread resulting from other market frictions.

What is the intuition behind the insurgence of a positive (negative) wedge when $l>0$ and $S_{t}^{C B} \neq S_{t}^{C B}(*)$ ? The basic idea is that if the truthful monetary authority announces it will intervene in the future, risk-neutral dealers revise their quotes for two specific reasons. First, as previously suggested, they have to increase (decrease) both the bid and the ask to ensure that, for an expected positive (negative) intervention, the market still clears, i.e., to ensure that their expected instantaneous inventory is still driftless, as in the monopoly case. Second, the competitive pressure among dealers obliges them to pass all extra revenues (costs) resulting from the potential arrival of a positive (negative) $\mathrm{CB}$ order onto the population of investors. The given elasticity of investors' demand for GBP then determines whether this effort eventually induces a positive or a negative wedge between the new bid and ask quotes. ${ }^{21}$ For $l=0$, as we have seen in Section 2.3, competition erodes completely the positive spread arising when dealers, holding significant market power, attempt to extract rents from the trading process. Indeed, $\Gamma=(1-q)\left(\bar{X}_{1}-\bar{X}_{2}\right)$ and $S_{2, t}-S_{1, t}=0$ when $l=0$. If instead $l>0$, further asymmetric quotes' revisions, thus a spread may be needed to transfer any additional cash inflow or outflow induced by the expected CB actions to the investors, because domestic and foreign currencydenominated bonds are not perfectly substitutable for risk-averse type 1 and type 2 traders.

Not surprisingly, as $\Gamma>0$, such wedge is always lower than the absolute spread set by a monopolistic dealer, $\frac{\pi}{2}\left(\bar{X}_{1}-\bar{X}_{2}\right)$, because of competition eroding the MMs' ability to extract rents from providing liquidity to the market. Therefore, the more binding is the no-profit condition, $E\left[\Pi_{t} \mid M_{t}\right]=0$, the smaller the competitive spread will be with respect to $\frac{\pi}{2}\left(\bar{X}_{1}-\bar{X}_{2}\right)$. Consequently, for a given and exogenous $X_{t}^{C B}$, the terms $\frac{\pi}{2}(\Gamma)$ and $-\frac{\pi q}{2(1-q)}(\Gamma)$ in $S_{1, t}$ and $S_{2, t}$, respectively, can be interpreted as the effect of the binding no-profit condition on the monopolistic quotes of Section 3.1. The wedge in Eq. (23) also depends crucially on the parameters controlling CB's optimal intervention schedule, because $X_{t}^{C B}$ is not exogenous in our model. For example, a more (less) intense trade-off between policy and wealth-preservation motives in the CB's loss function $L(\bar{S}, \lambda)$ may endogenously induce smaller (bigger) interventions, hence easing the pressure for competing dealers to even out expected cash inflows and outflows from trading.

Finally, the price at which the bid-ask wedge is equal to zero, $S_{t}^{C B}(*)$ in Eq. (24), is a weighted sum of the target rate $\bar{S}$ and the long-term fundamental value of the currency $f$, and is equal to the expected transaction price $S_{t}^{*}$. Therefore, if either the CB decides to pursue a more aggressive devaluation policy for the domestic currency or wealth-preservation becomes

\footnotetext{
${ }^{21}$ Observe incidentally that, in this model, the apparent arbitrage opportunity offered by a negative wedge cannot be exploited by the investors, as they arrive to the forex market just one at the time, can trade with the dealers just once, and do not return.
} 
more relevant in its loss function, its optimal intervention schedule $X_{t}^{C B}$ shifts upward and the MMs have to transact with the monetary authority (and the population of investors) at a higher price. The ensuing $\Delta S_{t}^{C B}(*)>0$ in fact prevents the dealers from retaining any additional cash inflow from $\mathrm{CB}$ trades, thus from having to impose a spread, while still clearing the market.

To clarify the interpretation of the multiple equilibria of Proposition 4, we parametrize the model for a $\mathrm{CB}$ chasing the trend and leaning against the wind, and report in Figure 2 the resulting spread and $X_{t}^{C B}$ for different $l \mathrm{~s}$. We start by setting the free parameter $S_{t}^{C B}$ equal to the target level $\bar{S}$. Later, we consider the issue of how sensitive is the analysis that follows to this assumption. Here we comment uniquely on the case of a CB chasing the trend (Figure 2a). The same reasoning however applies for a CB trying to resist that trend (Figure 2b). The positive likelihood of an intervention induces a positive spread, but such wedge is declining for increasing $l$, as so is the size of the positive expected CB order. As we suggested above, both bid and ask quotes go up in response to $X_{t}^{C B}>0$. The dealers need in fact to receive bigger sell orders and smaller buy orders from the investors to clear the market.

A positive expected CB order not only creates a potential imbalance in the dealers' inventory but also allows them to enjoy a potential increase of their net revenues at any point in time. Each single dealer would then try to modify slightly the bid and ask quotes, and the reservation rate for the $\mathrm{CB}$ order, in order to monopolize the trading flow while keeping the market clear. Competitive pressure from a multitude of dealers does eventually lead the quotes at steady state levels where both the expected instantaneous inventory drift $\left(-Z_{t}\right)$ and the expected instantaneous net revenues from trading $\left(\Pi_{t}\right)$ are equal to zero. The resulting spread is positive because, for the given set of parameters (and the implying investors' demand elasticity), those positive extra revenues from the CB have to be passed onto the population of investors, while $S_{t}^{C B}$ cannot be reduced, as it is fixed by assumption at $\bar{S}$. Indeed, at the given endowment ratio $\left(\frac{\bar{X}_{1}}{\bar{X}_{2}}=3\right)$ type 1 investors are bigger "net sellers" than otherwise identical type 2 investors are "net buyers." A positive spread therefore arises, for the dealers can achieve a net negative outflow of cash from the investors (that is $\left.(1-l) q X_{1, t} S_{1, t}+(1-l)(1-q) X_{2, t} S_{2, t}<0\right)$ to compensate the positive expected net cash inflow from the $\mathrm{CB}\left(l X_{t}^{C B} S_{t}^{C B}>0\right)$ just by revising upward the ask more than the bid.

Figure 3a displays the spread and net revenue components for investors of both types and the CB for increasing values of $\bar{X}_{1}$, hence of the endowment ratio. For higher $\bar{X}_{1}$, type 1 investors are more sensitive to changes in the exchange rate at which they would sell some GBP-denominated assets. Thus, the spread increases, as a smaller $\Delta S_{1, t}$ with respect to $\Delta S_{2, t}$ is needed to have a bigger sell order from type 1 investors and ensure that both the expected instantaneous drift and profit are equal to zero. Revenues from type 2 investors are steady, while the cash outflow from type 1 investors is bigger (in absolute terms) to compensate the inflow from the CB order.

It is worth observing that in this example $\left[q X_{1, t} S_{1, t}+(1-q) X_{2, t} S_{2, t}\right]<0$ : the competitive dealers are incurring an expected net loss (cash outflow) versus the population of investors to compensate for the gains (cash inflow) they are earning versus the CB. In other words, CB intervention is transferring rents to investors to induce them to hold more GBP-denominated bonds, but the dealers are unable to retain even part of these rents, as instead was happening in the case of a monopolist MM. However, if $S_{t}^{C B}$ is fixed at $S_{t}^{C B}(*)$, then the opposite effect occurs: even under extreme competitive pressure, dealers do not pass any of the costs or revenues from trading with the CB onto the population of investors. The higher potential price charged to 
the monetary authority lowers CB demand for GBP, and the absolute spread remains unchanged (at zero). If all three potential prices were allowed to change, then we would expect a smaller absolute spread than in the case of Figure 2a, but nonetheless different from zero. As expected, the intensity of these effects is reduced for increasing $l$, as it implies smaller CB orders.

How sensitive are the sign and size of the absolute spread in Figure 2a to the initial assumption that $S_{t}^{C B}$ is fixed at $\bar{S}$ ? To answer this question, we plot in Figure $3 \mathrm{~b}$ (for $l=0.03$ and $\frac{\bar{X}_{1}}{\bar{X}_{2}}=3$ ) the wedge of Eq. (23) for different values of $S_{t}^{C B}$, centered around $S_{t}^{C B}(*)$. Such wedge is negative for any $S_{t}^{C B}>S_{t}^{C B}(*)$. Why is this occurring? First, the fact that $X_{t}^{C B}$ is not particularly sensitive to changes in the rate at which CB's order is transacted, although the NFV of buying GBP $\left(f-R S_{t}^{C B}\right)$ is declining, suggests that, for our selected set of parameters, the wealth-preserving motives are weighted less than currency management in the loss function of Eq. (11). However, for higher $S_{t}^{C B}$, potential revenues from the $\mathrm{CB}$ are higher as well. Competitive dealers then need to suffer more cash outflows versus the population of investors, or need to make them more net sellers to pass to them these increasing rents, while still clearing the market. To do so, they raise more the bid quote, with respect to the ask, as type 1 investors are the needed net sellers. When $S_{t}^{C B}>S_{t}^{C B}(*)$, the quote revision on the bid becomes higher than on the offer, and a negative spread might be observed. Along the same lines, $S_{2, t}-S_{1, t}<0$ for any $S_{t}^{C B}>S_{t}^{C B}(*)$ if the CB were leaning against the wind.

The results of Proposition 4 convey very strikingly the intuition of imperfect substitutability as the only channel of effectiveness for CB intervention in a world of full information. It also suggests that, even when no information asymmetry among market participants is assumed, the impact of actions and announcements of the monetary authority on quotes and spreads is less trivial and more complicated than previously believed. Even a fully anticipated announcement (or arrival) of a CB order for the same size and sign may have different effects on quotes and spreads, depending not only on the degree of market power held by the dealers but also on the investors' current demand elasticity.

\subsection{A primer on the effectiveness of CB intervention}

We showed in the previous subsections that the introduction of a rational, but not necessarily profit-maximizing CB has a significant impact on the process of intraday price formation in the forex markets, even under the assumption of full information, when all risk-averse investors' orders do not arrive simultaneously but hit the dealers' screens one after the other. Indeed, one of the novelties of this paper is to extend the classic portfolio balance setting (with imperfect substitutability of domestic and foreign currency-denominated assets), previously restricted to a stylized market clearing just once, to the more realistic assumption of sequential trading. In particular, we showed that CB interventions may have both temporary and persistent effects on the exchange rate depending on $l$, the likelihood of the next order being from the CB.

Such effects are temporary because they disappears as soon as $l=0$, but may instead remain in the quotes for as long as the threat of future CB trades is significant and credible, i.e., for as long as $l>0$. Prices are initially revised because, at a specific point in time, dealers learn to face the arrival of a potential order by the monetary authority. Instantaneous market clearing forces them to effectively pass the order to incoming investors. As those investors are risk-averse, a risk premium is needed to compensate them for holding different positions from what they 
otherwise would if $l=0$. This adjustment is temporary if $l$ immediately goes back to zero, since compensation is no longer needed for new potentially arriving investors. The effect is instead persistent if $l$ remains positive for several trading rounds, as for each of them incoming investors have to be compensated for the possibility of having to rebalance their optimal portfolios.

The effects of CB actions on equilibrium quotes also depend crucially on the degree of market power held by the currency dealers. In the extreme scenario of a monopolist MM, the absolute spread is unchanged by CB intervention. It trivially ensues from Propositions 3 and 4 that the expected transaction price induced by $l>0$ is given by

$$
{ }^{M} S_{t}^{*}=\left(\frac{f}{R}-\pi X^{*}\right)+\frac{\pi L}{2 \pi L+\lambda R}\left(\bar{S}-\frac{f}{R}\right)+\frac{\pi}{2} X^{*}
$$

in the case of a monopolist $\mathrm{MM}\left({ }^{M} S_{t}^{*}\right)$, and by

$$
{ }^{C} S_{t}^{*}=\bar{S}+\frac{\lambda}{2 \pi L}\left(f-R S_{t}^{C B}\right)
$$

when instead dealers compete for the next incoming trade $\left({ }^{C} S_{t}^{*}\right)$. We measure the relative effectiveness of interventions under the two regimes by computing the variable $E M_{t}$ as

$$
E M_{t}=\left({ }^{M} S_{t}^{*}-\bar{S}\right)^{2}-\left({ }^{C} S_{t}^{*}-\bar{S}\right)^{2} .
$$

Positive values for $E M_{t}$ indicate that the threat of the arrival of CB trades pushes the expected transaction price closer to the objective $\bar{S}$ when the competitive scenario of Section 3.2 applies. We have then the following proposition.

Proposition 5 With full information and $l>0, C B$ interventions are maximally effective when $S_{t}^{C B}$ is equal to the risk-neutral price $\frac{f}{R}$, i.e., when the $N F V$ of currency trading $\left(f-R S_{t}^{C B}\right)$ is equal to zero for the monetary authority. $C B$ interventions are always more effective for competitive $M M s$ if wealth-preservation has no weight in the loss function $L(\bar{S}, \lambda)(\lambda=0)$. When instead $\lambda>0$, the same is true only if the absolute NFV of currency trading for the CB is "small" and/or if $\lambda$ is "small."

Proof. See the Appendix, where we also show that, if $\lambda$ is "small" or if there is no trade-off between wealth-preservation and policy motives in selecting $X_{t}^{C B}$, the $\mathrm{CB}$ is always better off when dealers compete for the incoming trade, i.e., $L^{C}(\bar{S}, \lambda)<L^{M}(\bar{S}, \lambda)$.

That the effectiveness of intervention is generally hindered by GBP trading being a positive NFV decision should not be surprising, given the attitude of our stylized CB toward final wealth in its loss function. Indeed, if wealth-preservation motives are conflicting with achieving the target $\bar{S}$, that trade-off shifts downward the CB's optimal intervention schedule for each $S_{t}^{C B}$. If instead wealth-preservation and policy motives reinforce each other, the resulting expected transaction price $S_{t}^{*}$ may overshoot $\bar{S}$. In addition, interventions tend to be more effective when the degree of dealers' market power is minimal. When such market power is significant, MMs' quest for profit maximization prevents interventions from being fully effective. The monopolist dealer in fact does not adjust his quotes completely in order to be able to extract some rents from the $\mathrm{CB}$, or to pass most of the costs of intervention onto investors. Consequently, the absolute 
size of the expected $X_{t}^{C B}$ is smaller, and the intervention is less effective. When instead there is a multitude of dealers, costs and revenues resulting from CB action are transmitted fully to the population of investors, and quotes' revisions are more substantial.

How general is this result? Speculative motives induce the CB to buy (sell) more or less foreign currency than it otherwise would to push $S_{t}^{*}$ toward $\bar{S}$. Hence, they may significantly affect the impact of the intervention schedule on the exchange rate. From Proposition 5 , it is clear that when wealth-preservation does not condition the CB's decision process $(\lambda=0)$ intervention is unequivocally more effective in the competitive scenario. In that case in fact the CB is a pure price-manipulator interested in managing the currency at any cost, thus offering the monopolist MM more opportunities to extract rents from the CB's activity, by not fully adjusting $S_{t}^{*}$ toward $\bar{S}$. Therefore, the resulting ${ }^{M} S_{t}^{*}$ is equal to a simple average of $\bar{S}$ and $S_{t}^{*, l=0}$, while ${ }^{C} S_{t}^{*}$ shifts promptly to $\bar{S}$.

When instead $\lambda>0$, there are some circumstances in which the reverse might be true (and $\left.E M_{t}<0\right)$. Proposition 5 tells us that this could occur when the role of wealth-preservation in the formulation of the optimal intervention schedule $X_{t}^{C B}$ is "very important," i.e., when it is "very profitable" to trade currencies and/or CB's loss function is "very sensitive" to the impact of its activity in the forex markets on its final wealth $W_{T^{\prime}}^{C B}$. In fact, when wealth-preservation motives are significant, either because the opportunity cost of not trading in currencies is "high" or because $\lambda$ is "big" (i.e., when our CB is less a price-manipulator and more a risk-neutral speculator), there is less room for the monopolist dealer to use his market power to extract rent from a more wealth-conscious monetary authority using its quotes. Moreover, when dealers compete for the next incoming trade, the smaller weight for policy motives in the CB's loss function is discounted integrally in bid and ask prices. This makes the resulting expected transaction price less responsive to the target level $\bar{S}$.

Is the impact of different degrees of market power on the distance between $S_{t}^{*}$ and $\bar{S}$ economically significant? As an additional example, we report in Figure 4 the effect of a CB chasing the trend on the expected transaction price as a function of the likelihood of intervention $l$ for the two extreme cases of monopolist and competitive MMs. Quotes and intervention sizes for the competitive case are evaluated at $S_{t}^{C B}(*)$, i.e., at the rate at which the bid-ask spread is zero, for the results of the simulation not to be affected by arbitrary choices for the free parameter $S_{t}^{C B}$. Because $\bar{S}>\frac{f}{R}-\pi X^{*}$, the CB is buying GBP in the attempt to induce a devaluation of the dollar. The expected transaction price ${ }^{C} S_{t}^{*}$ gets closer to the target level $\bar{S}$, although initially with some overshooting at low $l \mathrm{~s}$, and converges to it for higher values of $l$. In a market where there is a monopolist dealer, the intervention is least effective. Indeed, ${ }^{M} S_{t}^{*}$ undershoots the target level and never approaches it for increasing $l$.

At the same time, the absolute size of the endogenously determined CB order tends to be higher in the competitive scenario, but the difference shrinks when the arrival of such order is more likely. This occurs because $S_{t}^{C B}(*)$ (Eq. (24)) is lower than $S_{t}^{C B}$ in Eq. (18), hence the expected NFV of buying GBP-denominated bonds and selling dollar-denominated T-bills $\left(f-R S_{t}^{C B}\right)$ is higher. In other words, when dealers compete for the incoming trade, and $l$ and $S_{t}^{C B}$ are low, wealth-preservation reinforces the CB's resolve to weaken the domestic currency. Overshooting of ${ }^{C} S_{t}^{*}$ with respect to $\bar{S}$ may ensue, as in this case. When instead dealers' market power is significant, $f-R S_{t}^{C B}$ is smaller and may even become negative, thus inducing a tradeoff between the pursuit of $\bar{S}$ and speculation. Consequently, ${ }^{M} S_{t}^{*}$ undershoots $\bar{S}$. Although 
empirical evidence on the relationship between effectiveness of intervention and degree of market power is scarce, and mostly anecdotal, there appears to be consensus over the fact that more frequent, more successful, and larger interventions are observed for heavily traded currencies in quasi-competitive market settings, with respect to the case of less intermediated exchange rates of emerging economies. ${ }^{22}$

\section{Information asymmetry}

We have proceeded so far in a world of full information. This restrictive assumption allowed us to explore a single channel through which intervention affects transaction prices, imperfect substitutability, and to focus on its impact on quotes and spreads. Full information prevented CB intervention from having any information content. Hence, it prevented us from examining the information asymmetry channel of currency management effectiveness. Through this second channel, according to the economic literature mentioned in Section 1, actions by the monetary authority, by conveying fundamental information and thus affecting the market's beliefs and their dispersion, may have a more persistent effect on quotes and, we add, spreads, beyond the revisions induced by portfolio balance considerations. In this section we fill this gap by introducing information asymmetry related to CB intervention, in particular by introducing uncertainty about the Central Bank's objective $(\bar{S})$, the fundamental value of the currency $(f)$, and the likelihood of intervention $(l) .^{23}$

In particular, we examine a stylized case in which all those forms of uncertainty interact, and study their combined impact on quotes and spreads with respect to the two benchmark cases of no intervention (Proposition 1) and intervention with full information (Section 3). Although the main focus of our analysis is on the effects of information asymmetry between dealers (and investors) and the CB, we also consider the possibility that (as suggested by Goodhart and Hesse (1991) and Lewis (1995), among others) not all agents are able to immediately observe the arrival of an intervention, or its magnitude. This implies that investors may update their beliefs with a delay with respect to the dealers, and just after having observed the new resulting transaction prices. Hence, heterogeneity of otherwise identical beliefs may arise.

To deal with information asymmetry, we need some additional terminology and few additional assumptions. We define $M_{t}, I_{t}$, and $\mathbf{z}_{t}$ as the information sets available to dealers, investors, and $\mathrm{CB}$ at $t$, respectively. $I_{t}$ and $\mathbf{z}_{t}$ contain all past transaction prices $S_{1, t-j}$ or $S_{2, t-j}$ and the corresponding orders $X_{i, t-j}$, while $M_{t}$ also includes units of foreign currency traded by the CB up to (but excluding) time $t .^{24}$ Because our stylized dealers do not maximize expected utility of future wealth, they do not need to formulate beliefs about the future fundamental value of the

\footnotetext{
${ }^{22}$ See, for example, Brown (2000) and Chancellor (2000) for anecdotal evidence on the actions of Asian monetary authorities during the crisis of 1997 and 1998, and Moloney (2000) on interventions by G-7 CBs over the past 25 years.

${ }^{23} \mathrm{CBs}$ may not, and usually do not declare explicitly the degree of resolution of their trade-off between policy and wealth-preserving motives $(\lambda)$ as well. We explored the issue of uncertainty surrounding $\lambda$ in a previous version of this paper, and found that its implications for quotes and spreads were substantially similar to those deriving from uncertainty surrounding $\bar{S}$ and $f$.

${ }^{24}$ This occurs because investors observe the rate at which their order at time $t$ is going to be transacted, $S_{i, t}$, only after they arrive to the dealers with their demand schedule $X_{i, t}$, according to the exogenous orderly point process $G(t)$.
} 
currency. However, being in the business of making and clearing the market, they need to learn investors' beliefs about $f$. For sake of simplicity, we assume that dealers know exactly those beliefs, and so does the $\mathrm{CB},{ }^{25}$ but still formulate their own beliefs about $f$ to estimate the sign and size of the policy and wealth-preserving components in the incoming $\mathrm{CB}$ order.

As a result of information asymmetry surrounding $\bar{S}, f$, and/or $l$, the actions of the monetary authority can surprise the market for their direction and magnitude (thus being unexpected, along the lines of Naranjo and Nimalendran (2000)), and/or for their timing (thus being unannounced). Therefore, as in Section 3.4, in the reminder of the paper we also study the effectiveness of the announcement of an intervention and of its eventual arrival on the exchange rate, in terms of its speed of adjustment toward the target level, in terms of its proximity to that target, and finally in terms of its persistence around it over time.

\subsection{The extended model}

We start by providing a two-step stylized representation of the underlying economic process leading to $\mathrm{CB}$ intervention: we assume that at $t=0$ nature first exogenously chooses $f$, the mean long-term value of the currency, between $f_{H}$ and $f_{L}\left(f_{H}>f_{L}\right)$, with probability $p_{f}$ and $\left(1-p_{f}\right)$, respectively, then picks the two parameters controlling the currency management policy of the monetary authority, the target level $\bar{S}$, between $\bar{S}_{H}$ and $\bar{S}_{L}\left(\bar{S}_{H}>\bar{S}_{L}\right)$, and the likelihood of intervention $l$. If nature chooses $f_{H}\left(f_{L}\right)$, then $\bar{S}=\bar{S}_{H}\left(\bar{S}=\bar{S}_{L}\right)$ with probability $\psi$ known to all market participants. Hence, the parameter $\psi$ can be interpreted as a measure of the correlation between the fundamental value of the currency and the currency management policy selected by the $\mathrm{CB}$, or alternatively of the consistency of that policy with the basic economic factors driving the long-term behavior of the exchange rate. For $\psi>\frac{1}{2}$ there is a positive "true" correlation between $f$ and $\bar{S}$, i.e., the pairs $\left(f_{H}, \bar{S}_{H}\right)$ and $\left(f_{L}, \bar{S}_{L}\right)$ are more likely than $\left(f_{H}\right.$, $\left.\bar{S}_{L}\right)$ and $\left(f_{L}, \bar{S}_{H}\right)$, respectively. A $\psi<\frac{1}{2}$ instead implies that $f$ and $\bar{S}$ are negatively correlated. Finally, if $\psi=\frac{1}{2}$ it is equally probable that, for a given $f, \bar{S}$ is going to be high or low, i.e., the fundamental value of the exchange rate and the policy motive in the CB's loss function are ex ante uncorrelated.

We also assume that the CB, aware of $\bar{S}$ and $l$, is going to be better informed about nature's choice for $f$ than the rest of the market just with probability $v$. This implies that not in all circumstances $\mathrm{CB}$ trades may be informative about $f$. In fact, with probability $(1-v)$, the CB formulates its intervention based on the true $\bar{S}$ and on its belief about what $f$ should be, $E\left[f \mid \mathbf{z}_{t}^{U}\right]$, where $\mathbf{z}_{t}^{U}$ is the information set of the uninformed $\mathrm{CB}^{26}$ When uninformed, the monetary authority knows that no trade can be informative about $f$, hence it never updates its original expectation after observing the flow of executed trades and the corresponding transaction prices. Investors and dealers do not observe any of the variables chosen by nature, hence formulate their orders and quotes, respectively, based on their beliefs about $p_{f}, p_{\bar{S}}$, and $l$. We could then also interpret $\psi$ as controlling for the amount of uncertainty surrounding CB's activity in the

\footnotetext{
${ }^{25}$ Therefore, Proposition 2 implies that its optimal intervention schedule is now given by $X_{t}^{C B}=$ $\gamma\left[\bar{S}-\left(\frac{E\left[f \mid I_{\mathrm{t}}\right]}{R}-\pi X^{*}\right)+\frac{\lambda}{2 \pi L}\left(f-R S_{t}^{C B}\right)\right]$.

${ }^{26}$ In other terms, CB trades depend ex post on the "true" mean $f$ selected by nature just with probability $v$. The information set of the informed CB is then given by $\mathbf{z}_{t}^{I}=\left\{\mathbf{z}_{t}^{U}, f\right\}$. Furthermore, we impose the logic restriction that the uninformed $\mathrm{CB}$ cannot use its knowledge of $\bar{S}$ to infer $f$, i.e., that $E\left[f \mid \mathbf{z}_{t}^{U}\right]$ is given.
} 
currency market in the eyes of other market participants. In fact, when $\psi \neq \frac{1}{2}$ investors and MMs can more easily infer what $\bar{S}$ is from their expectations about $f$.

Investors use their beliefs on $f$ to formulate their optimal demand schedules $X_{i, t}$. As already mentioned, we assume that both dealers and the monetary authority know perfectly investors' beliefs $E\left[f \mid I_{t}\right]$, hence their incoming orders $X_{i, t}$. This hypothesis, while simplifying the analysis that follows, allows us to concentrate on the information asymmetry between dealers (and investors) and the CB. Nonetheless, dealers' beliefs about $f$ are not irrelevant in the model. Indeed, dealers also need to predict what the informed CB's intervention is going to look like, when and if it occurs. Hence they need to formulate some beliefs about $f$ to be able to forecast $X_{t}^{C B}$. Consistently with available (albeit scarce) empirical evidence (e.g., Fischer and Zurlinden (1999)), in our setting all dealers observe sign and size of the most recent CB transaction, know that this trade may be informative about $f$, and use this information to update their priors on it. Vice versa, as in most currency markets, investors know if an intervention occurred, but do not observe the transacted amounts. This lack of transparency slows down their process of learning about $f$ from the trade flow. We accommodate such a circumstance by assuming that investors observe the most recent order $X_{i, t+1}$ arrived immediately after the intervention occurred (at time $t$ ) and the price $S_{i, t+1}$ at which it was transacted, that they infer from the sign of the observed quote revision $S_{i, t+1}-S_{i, t-j}$ which kind of intervention has taken place, and that they eventually update their beliefs based on this filtered information, although with a delay. This form of order flow uncertainty is between dealers and investors, and not among dealers, as in Evans and Lyons (2000), and has not been previously explored by the currency microstructure literature. ${ }^{27}$

It is clear from an examination of the demand function of an informed CB that both the sign and the magnitude of the intervention are going to depend crucially on the realizations of $f$ and $\bar{S}$. For sake of simplicity, we assume that $\lambda$ is small enough for wealth-preservation not to be the dominating concern for the monetary authority, and that $\bar{S}$ and $f$ control the direction and size, respectively, of its optimal intervention, regardless of $l$. In particular, we consider the case in which, for a low enough $\lambda$, the long-term value of the currency $f_{H}\left(f_{L}\right)$ is such that the expected NFV of investing in GBP-denominated bonds for the informed $\mathrm{CB},\left(f-R S_{t}^{C B}\right)$, is positive (negative). This assumption de facto restricts that $\mathrm{CB}$ to four distinct interventions for magnitude and direction. For high (low) values of both $\bar{S}$ and $f$, there is no trade-off between policy and wealth-preservation motives in $L(\bar{S}, \lambda)$, as they both push the monetary authority to buy (sell) the foreign currency. The resulting intervention is then endogenously big in size, ${ }_{B} X_{t}^{C B}$. For low (high) values of $\bar{S}$ and high (low) values of $f$, a trade-off between policy and speculation motives instead exists, as the informed CB wants to chase the trend (lean against the wind) while selling (buying) GBP is a negative NFV action. However, the low $\lambda$ ensures that the second effect does not dominate the first, and that eventually the CB sells (buys) small amounts of GBP, ${ }_{S} X_{t}^{C B} \cdot{ }^{28}$ Hence, when $\psi$ is high (low), $f$ is positively (negatively) correlated with $\bar{S}$, the trade-off in CB's loss function $L(\bar{S}, \lambda)$ is more (less) significant, hence the informed $\mathrm{CB}$ is more likely to intervene with a big (small) order.

What is the optimal intervention strategy of the uninformed CB? We focus on a specific

${ }^{27}$ One exception is the empirical study of Peiers (1997), who applies Granger causality tests to DEMUSD quotes and shows that Bundesbank trades are revealed first to dealers and then to the general public.

${ }^{28}$ If, for example, $\bar{S}=\bar{S}_{L}$ and $f=f_{H}$, the monetary authority leans against the wind $\left(\right.$ as $\left.\bar{S}_{L}<\frac{f_{H}}{R}-\pi X^{*}\right)$ by selling GBP, i.e., $X_{t}^{C B}<0$; however, at $f_{H}$ the NFV of investing in GBP-denominated bonds is positive, and induces the $\mathrm{CB}$ to reduce the amount of GBP sold to dealers. 
pooling equilibrium in which the uninformed monetary authority, aware of the true $\bar{S}$ but not of the true $f$, always buys (sells) foreign currency by the amount ${ }_{S} X_{t}^{C B}>0\left({ }_{S} X_{t}^{C B}<0\right)$ when $\bar{S}=\bar{S}_{H}\left(\bar{S}=\bar{S}_{L}\right)$. This mimicking behavior is interesting to us because it prevents observed interventions from being fully revealing of the information available to the CB about $f$, i.e., because it introduces uncertainty in the learning process of the dealers when they transact with the CB. This uncertainty clearly depends on $v$, the parameter controlling the CB's skills at understanding fundamentals. More specifically, the optimal intervention schedules of the informed $\left({ }^{I} X_{t}^{C B}\right)$ and uninformed $\left({ }^{U} X_{t}^{C B}\right) \mathrm{CB}$ are given by

$$
{ }^{I} X_{t}^{C B}=\left\{\begin{array}{cl}
{ }_{B} X_{t}^{C B}>0 & \text { if nature chooses }\left(\bar{S}_{H}, f_{H}\right) \\
{ }_{S} X_{t}^{C B}>0 & \text { if nature chooses }\left(\bar{S}_{H}, f_{L}\right) \\
{ }_{S} X_{t}^{C B}<0 & \text { if nature chooses }\left(\bar{S}_{L}, f_{H}\right) \\
{ }_{B} X_{t}^{C B}<0 & \text { if nature chooses }\left(\bar{S}_{L}, f_{L}\right)
\end{array}\right.
$$

and, for the chosen $E\left[f \mid \mathbf{z}_{t}^{U}\right]$,

$$
{ }^{U} X_{t}^{C B}=\left\{\begin{array}{cl}
{ }_{S} X_{t}^{C B}>0 & \text { if nature chooses } \bar{S}_{H} \text { for any true } f \\
{ }_{S} X_{t}^{C B}<0 & \text { if nature chooses } \bar{S}_{L} \text { for any true } f,
\end{array}\right.
$$

respectively, when MMs use the following pricing schedule:

$$
S_{t}^{C B}=\left\{\begin{array}{cc}
{ }^{M} S_{t}^{C B},{ }^{C} S_{t}^{C B} & \text { for any order }{ }_{S} X_{t}^{C B},{ }_{B} X_{t}^{C B} \\
0 & \text { for any order } X_{t}^{C B}<0\left(X_{t}^{C B} \neq{ }_{S} X_{t}^{C B}\right) \\
\infty & \text { for any order } X_{t}^{C B}>0\left(X_{t}^{C B} \neq{ }_{S} X_{t}^{C B}\right) .
\end{array}\right.
$$

We report in the Appendix a set of restrictions on the dispersion of the model's parameters that, together with the exchange rates in Eq. (30) and the results of Proposition 2, ensure that those $\mathrm{CB}$ actions are indeed optimal, in particular that the uninformed CB will pool with the informed CB's ${ }^{I} X_{t}^{C B}$ not only in sign but also in magnitude, for any true $f$ selected by nature. Intuitively, the model's parameters have to be such that policy motives are significant enough to determine the direction of intervention for both the informed and the uninformed CB. The extreme-pricing hypothesis of Eq. (30) guarantees that, if the uninformed CB decides to pool with the informed one, the small positive order executed with the dealer(s) is equal to ${ }_{S} X_{t}^{C B}>0$. In fact, $S_{t}^{C B}$ in Eq. (30) is "too low" or "too high" when the optimal ${ }^{U} X_{t}^{C B}$ is negative or positive, respectively, given its prior $E\left[f \mid z_{t}^{U}\right]$, therefore making mimicking optimal for the uninformed $\mathrm{CB}$ at the true $\bar{S}$. In this respect, we can then interpret the parameter $v$ also as controlling for the degree of informativeness of CB trading. If $v=1$, any intervention can be attributed to the informed CB, hence is fully revealing not only about $\bar{S}$ but also about $f$. Our dealers can in fact infer from Eq. (14) and from the observed transaction sign and magnitude what nature's choice for $f$ and $\bar{S}$ were, and revise their beliefs accordingly. If instead $v=0$, CB trading is never informative about $f$. Consequently, no updating of beliefs about $f$ ever occurs.

\subsection{Beliefs and beliefs' revisions}

Dealers choose potential prices and investors formulate their demand for the foreign currency based on their beliefs about $f, \bar{S}$, and $l$. In this subsection we describe how these beliefs are 
updated over time in our model. We first focus on the dealers. At each point in time $t$, before setting their potential prices and before any new order arrives to the market, the MMs have some priors about the true probability of nature having chosen $f_{H}\left(q_{f, t}\right)$ and $\bar{S}_{H}\left(q_{\bar{S}, t}\right)$ at $t=0$, where $q_{f, t}=\operatorname{Pr}\left\{f=f_{H} \mid M_{t}\right\}$ and

$$
q_{\bar{S}, t}=\operatorname{Pr}\left\{\bar{S}=\bar{S}_{H} \mid M_{t}, q_{f, t}\right\}=q_{f, t} \psi+\left(1-q_{f, t}\right)(1-\psi)
$$

Therefore, the MMs' expected values for $f$ and $\bar{S}$ at time $t$ are given by $E\left[f \mid M_{t}\right]=q_{f, t} f_{H}+$ $\left(1-q_{f, t}\right) f_{L}$ and $E\left[\bar{S} \mid M_{t}\right]=q_{\bar{S}, t} \bar{S}_{H}+\left(1-q_{\bar{S}, t}\right) \bar{S}_{L}$, respectively. At the beginning of the trading period $[0, T]$ the dealers have also priors about $l\left(E\left[l \mid M_{0}\right]\right)$. This prior may depend on their past experience, or on the announcement of a future intervention by the monetary authority. Hence, we can interpret the difference between $E\left[l \mid M_{0}\right]$ and the true $l$ and the dispersion of dealers' beliefs around it $\left(\operatorname{Var}\left[l \mid M_{0}\right]\right)$ as measures of the credibility of CB's announcements in the currency market, and analyze the impact of different degrees of such credibility on quotes and spreads. If $f_{M_{\mathrm{t}}}(l)$ is the prior distribution of $l$ given the information available to the MMs at time $t$, before any new order arrives, then dealers' prior about $l$ is given by

$$
q_{l, t}=E\left[l \mid M_{t}\right]=\int_{0}^{1} l f_{M_{\mathrm{t}}}(l) d l .
$$

We assume that those priors are updated according to Bayes' Rule every time a new order arrives to the market. The following proposition summarizes the way dealers' beliefs $q_{l, t}, q_{f, t}$, and $q_{\bar{S}, t}$ evolve into the corresponding posteriors $p_{l, t}, p_{f, t}$, and $p_{\bar{S}, t}$ in this stylized economy.

Proposition 6 The potential arrival of investors' orders (a CB order) at $t$ induces a downward (upward) revision of the dealers' prior $q_{l, t}$ into $p_{l, t}$, where

$$
p_{l, t}=\left\{\begin{array}{cc}
E\left[l \mid M_{t}\right]-\frac{\operatorname{Var}\left[l \mid M_{\mathrm{t}}\right]}{1-E\left[l \mid M_{\mathrm{t}}\right]}<E\left[l \mid M_{t}\right] & \text { if } X_{i, t} \text { arrives } \\
E\left[l \mid M_{t}\right]+\frac{\operatorname{Var}\left[l \mid M_{\mathrm{t}}\right]}{E\left[l \mid M_{\mathrm{t}}\right]}>E\left[l \mid M_{t}\right] & \text { if }{ }_{S} X_{t}^{C B} \text { arrives } \\
l & \text { if }{ }_{B} X_{t}^{C B} \text { arrives }
\end{array}\right.
$$

The posterior $p_{l, t}$ is used by the dealers for their reservation quotes at time $t$, and becomes their best prior at time $t+1$, i.e., $q_{l, t+1}=p_{l, t}$. When an intervention occurs at time $t$, its sign is always fully revealing of the $C B$ 's target $\bar{S}$. The arrival of a big positive $\left({ }_{B} X_{t}^{C B}>0\right)$ or of a big negative $\left({ }_{B} X_{t}^{C B}<0\right) C B$ order induces full revelation to the dealers of the true $f$ (hence of the true l). If a small positive intervention $\left({ }_{S} X_{t}^{C B}>0\right)$ occurs, then dealers update their beliefs about $f$ according to:

$$
q_{f, t+1}=p_{f, t}=\frac{q_{f, t}(1-v)}{q_{f, t}(1-v)+\left(1-q_{f, t}\right) v},
$$

while if a small negative intervention $\left({ }_{S} X_{t}^{C B}<0\right)$ occurs, we have

$$
q_{f, t+1}=p_{f, t}=\frac{q_{f, t} v}{q_{f, t} v+\left(1-q_{f, t}\right)(1-v)} .
$$


Proof. See the Appendix.

That the sign of $X_{t}^{C B}$ is a sufficient statistic for the MMs to learn about $\bar{S}$ follows immediately from our assumption that the NFV of currency trading $\left(f-R S_{t}^{C B}\right)$ is always positive (negative) for $f_{H}\left(f_{L}\right)$. In addition, the arrival of ${ }_{B} X_{t}^{C B}>0$ or ${ }_{B} X_{t}^{C B}<0$ is fully revealing of $f$ as well. When instead ${ }_{S} X_{t}^{C B}>0\left({ }_{S} X_{t}^{C B}<0\right)$ arrives, the possibility that it was submitted by an uninformed CB induces just a partial revision of the dealers' priors for $f$. More interestingly, because of the potential informativeness of $\mathrm{CB}$ actions about $f$, not only the likelihood of an intervention but the intervention itself, when and if it occurs, has an impact on MMs' beliefs, thus eventually on quotes and spreads. This effect persists even when $l$ drops to zero. A CB trade, because potentially informative, induces a permanent revision of dealers' expectations about $f$, and such revision is embedded in their reservation prices, as we will see more clearly in the remainder of this section. This is the information asymmetry channel of effectiveness of intervention.

Proposition 6 also states that the arrival of $X_{i, t}\left({ }_{S} X_{t}^{C B}\right)$ reduces (increases) the perceived likelihood of a future action by the monetary authority. The effect of the potential arrival of an investor or a CB on MMs' beliefs is crucial in our model. Dealers set potential prices conditional to the arrival of an investor of type 1, an investor of type 2, or the CB. The likelihood of such arrivals $((1-l) q,(1-l)(1-q)$, and $l$, respectively) is by assumption exogenous, i.e., independent from those quotes. If an investor arrives, then the dealers are induced to believe that an intervention is less likely, and the posterior about $l, p_{l, t}=E\left[l \mid M_{t}, X_{i, t}\right]$, is revised downward. If instead the CB hits the dealers' screens with a small order, the posterior about $l, p_{l, t}=E\left[l \mid M_{t}, X_{t}^{C B}\right]$, is revised upward. Therefore, the potential bid or ask prices already discount the knowledge of that information, i.e., are computed based on $p_{l, t}$ and not $q_{l, t}$. The same is true when the monetary authority intervenes.

The entity of these updates depends on the degree of dispersion in the dealers' priors about $l, \operatorname{Var}\left[l \mid M_{t}\right]$. Widely dispersed beliefs around $l$, for a given $E\left[l \mid M_{t}\right]$, induce a bigger impact of order arrivals on beliefs' revisions. As suggested above, we can interpret $\operatorname{Var}\left[l \mid M_{t}\right]$ as a measure of the degree of credibility enjoyed by a $\mathrm{CB}$ when it announces that it will intervene in the future. When the monetary authority divulges information to the market about its future actions, MMs form an opinion on $l, E\left[l \mid M_{t}\right]$. Even if $E\left[l \mid M_{t}\right]=l$, i.e., even if the dealers are able to predict the true probability of intervention, the uncertainty in that estimate is going to affect the size of their beliefs' updates (and quotes' revisions) during trading. Thus, depending on their perceived credibility, CB's announcements have a potentially significant impact not only on price levels but also on their variance. The arrival of ${ }_{B} X_{t}^{C B}$, by fully revealing $f$ and $\bar{S}$, induces a new $q_{l, t}=l$, for the magnitude of the intervention depends on its likelihood as well as on policy and wealth-preservation motives. However, because of the assumed mimicking behavior by the uninformed CB, observing the sign of a small CB trade does not reveal the true likelihood of intervention. The dealers then use Eq. (33) to generate a new posterior for $l$. In both cases, we assume that the resulting value for $l$ ( or $p_{l, t}$ ) becomes dealers' best prior for the likelihood of the next incoming agent being a Central Bank, to reflect the fact that, following a governmentoriginated transaction, the likelihood of future interventions tends usually to decline, as reported, for example, in Fischer and Zurlinden (1999).

How do investors change their beliefs about $f$ when the CB intervenes? Dealers observe first-hand $X_{t}^{C B}$, hence they can revise their expectations about $f$ using Bayesian updating. We 
previously suggested that investors in our economy are only aware that an intervention actually happened, if announced, but do not observe size and direction of that transaction. We also assume that investors are unaware of past and current dealers' beliefs about $f, \bar{S}$, and $l .{ }^{29}$ However, we assume that they do observe every past transaction price $S_{i}$ at which other investors in the market might have traded GBP, and the corresponding amount $X_{i}$. Investors also know that the CB might not be better informed than the rest of the market about $f$. Hence, they revise upward (downward) their expectations about $f$ if there is a positive (negative) drift in the most recent transaction rate at the bid or at the offer, $S_{i, t+1}$, after the intervention occurred. Therefore, transaction prices convey (albeit noisy) information to otherwise less informed agents in the economy. If we define investors' priors for $p_{f}$ as ${ }^{I} q_{f, t}=\operatorname{Pr}\left\{f=f_{H} \mid I_{t}\right\}$, the following proposition applies.

Proposition 7 When an intervention occurs, investors observe the positive (negative) change in the first transaction price $S_{i, t+1}$ registered after the intervention (but not dealers' new posteriors and uninformed $C B$ 's priors) and revise their beliefs at $t+2$ about $f$ according to

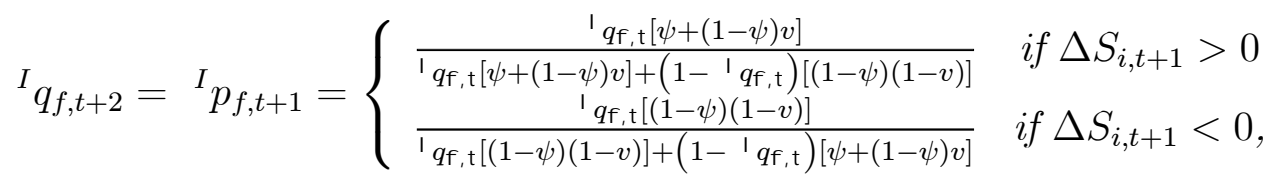

if the first transaction after the time $t$ intervention happens at $t+1 ; \Delta S_{i, t+1}=S_{i, t+1}-S_{i, t-j}$ is the revision in the bid $(i=1)$ or ask $(i=2)$ price, and $t-j$ is when the latest pre-intervention transaction on the side $i$ of the market occurred.

Proof. See the Appendix.

Investors use Bayes' Rule to formulate a new expectation for $f$ based on the observed quote revision because they are assumed to be unaware of dealers' past priors and posteriors for $\bar{S}, f$, and $l$, and of the uninformed CB's belief $E\left[f \mid z_{t}^{U}\right]$. More specifically, in Proposition 7 investors revise their beliefs about $f$ simply using the information potentially conveyed by the sign of the change in the transaction price, hence using a less refined update structure than the one our dealers rely upon after observing the magnitude of the CB trade. Consequently, dealers and investors may disagree on $f$ even once such trade took place, i.e., it is possible that ${ }^{I} p_{f, t+1} \neq p_{f, t}$. Moreover, investors revise their beliefs just when a transaction occurred after the intervention by the monetary authority. This delay induces positive serial correlation in transaction prices immediately following the arrival of a CB order, an easily testable implication of our model.

\section{A particular day in the currency market}

Armed with these results, in the next two subsections we investigate how dealers with different degrees of market power revise their quotes when investors' orders and CB interventions arrive to

\footnotetext{
${ }^{29}$ This assumption prevents our investors from being able to infer from observed past transaction prices and quotes following CB's intervention at time $t, S_{i, t+j}$, what exactly the new dealers' posterior for $f, p_{f, t}$, is going to be. Hence, we abstract in this paper from the issue of agents estimating other agents' expectations from the order flow, as explored in depth by Foster and Viswanathan (1996).
} 
our stylized forex market with information asymmetry, and examine the resulting behavior of the expected transaction price $S_{t}^{*}$ during the interval $[0, T]$. For both a monopolist and competitive MMs, we specify the following sequence of events (stages) during the trading day:

0. At $t=0 \mathrm{MMs}$ set potential bid and ask quotes based on their initial expectations for $f, \bar{S}$, and $l$. If the resulting expected likelihood of intervention is positive $\left(q_{l, 0}>0\right)$, such quotes are revised with respect to the benchmark scenario of Proposition 1;

1. Order flow from investors $\left(X_{i, t}\right)$ starts arriving. When this occurs, MMs do not revise their beliefs about $f$ and $\bar{S}$, because they know investors are not better informed than they are about $f$. However, dealers revise their prior about $l$ according to Proposition 6 . This revision induces a change in the quotes and spreads with respect to the levels of stage 0 . Following the resulting change in $S_{t}^{*}$, investors do not revise $E\left[f \mid I_{t}\right]$, because they know that no CB intervention has yet occurred;

2. When the monetary authority eventually intervenes, both dealers and investors know the $\mathrm{CB}$ has arrived. However, MMs transact directly with the government. Hence, they observe sign and magnitude of its intervention, update their beliefs about $\bar{S}, f$, and $l$ (as described in Section 4.2), and execute the order of the monetary authority at a price $S_{t}^{C B}$ that contains all the resulting revealed information. Such information is then discounted in their reservation bid and ask quotes for any future incoming order. As our stylized forex market is semi-strong efficient, this process of beliefs' and quotes' revision occurs only if the actual intervention was unexpected in its direction and size $\left(\bar{S} \neq E\left[\bar{S} \mid M_{t}\right]\right.$ and/or $\left.f \neq E\left[f \mid M_{t}\right]\right)$, or if its arrival was unannounced $\left(l \neq E\left[l \mid M_{t}\right]\right)$;

3. The first investors' order coming to the market after the intervention is processed at the new bid or ask price. After this trade occurs, investors observe the corresponding transaction price change $\Delta S_{i, t+1}$ and revise their beliefs about $f$ according to Proposition 7;

4. Incoming investors use their new expectation for $f$ to formulate their demand for GBP. Dealers use investors' new $E\left[f \mid I_{t, \Delta S_{\mathrm{i}, \mathrm{t}+1}}\right]$ to update their potential bid and ask quotes from the levels of stage 3 ;

5. All future orders $X_{i, t}$ do not induce any additional update of investors' and dealers' priors about $f$ (and $\bar{S}$ ), but just of MMs' priors for $l$, unless the CB credibly announces that it is not going to intervene in the future. Hence, observed transaction prices may still change from the immediately preceding round of trading.

Before the intervention actually occurs, the impact of $q_{l, t}>0$ on $S_{t}^{*}$ is due exclusively to the imperfect substitutability of dollar versus GBP-denominated bonds for risk-averse investors. The arrival of a $\mathrm{CB}$ order may instead reveal new information about $f$ and $\bar{S}$. At different stages, both dealers and investors update their priors based on such information. By the end of the trading day, if the perceived likelihood of a future intervention by the monetary authority drops to zero, any residual effect on $S_{t}^{*}$ with respect to $S_{0}^{*, l=0}$ should be due exclusively to the signaling effect of the past intervention. In the reminder of the paper we analyze the process of intraday price formation implied by the above stages over the interval $[0, T]$ for a monopolist 
and competitive MMs and simulate the resulting equilibrium for a basic parametrization of the economy. ${ }^{30}$

\subsection{The monopolist dealer}

In this subsection we derive, at each point in time $t_{n} \in[0, T]$ across each stage of the sequence of the sequence of events above described, explicit solutions for the problem of a monopolist dealer (Eq. (15)) when his expectations $E\left[f \mid M_{t_{n}}\right], E\left[\bar{S} \mid M_{t_{n}}\right]$, and $E\left[l \mid M_{t_{n}}\right]$ replace $f, \bar{S}$, and $l$ and the investor's optimal demand (Eq. (3)) depends on $E\left[f \mid I_{t_{n}}\right]$ (instead of $f$ ). We start by assuming that at time zero the monetary authority announces it will intervene in the future, but that, as it often happens, the CB is vague about the objectives of its potential action. ${ }^{31}$ Based on this information, and on his past experience, our dealer formulates a set of priors for $l$ and $f, q_{l, 0}$ and $q_{f, 0}$, and computes $q_{\bar{S}, 0}$ using $\psi\left(q_{\bar{S}, 0}=q_{f, 0} \psi+\left(1-q_{f, 0}\right)(1-\psi)\right)$. Investors formulate their own beliefs about $p_{f},{ }^{I} q_{f, 0}$, and those beliefs are assumed to be perfectly known to the dealer. As we have done so far, we focus here on the sign and magnitude of the quote revision induced at each of the stages with respect to the benchmark scenario of no intervention $(l=0)$ of Proposition 1.

At stage 0, before any order arrives, the dealer revises the potential bid and ask quotes with respect to $S_{1,0}^{l=0}$ and $S_{2,0}^{l=0}$,

$$
\begin{aligned}
& S_{1,0}=S_{1,0}^{l=0}+\Delta^{M} S_{0}^{*}=S_{0}^{*, l=0}-\frac{\pi(1-q)}{2}\left(\bar{X}_{1}-\bar{X}_{2}\right)+\Delta^{M} S_{0}^{*} \\
& S_{2,0}=S_{2,0}^{l=0}+\Delta^{M} S_{0}^{*}=S_{0}^{*, l=0}+\frac{\pi q}{2}\left(\bar{X}_{1}-\bar{X}_{2}\right)+\Delta^{M} S_{0}^{*},
\end{aligned}
$$

respectively, where $S_{0}^{*, l=0}=\frac{E\left[f \mid I_{0}\right]}{R}-\pi X^{*}$ and $\Delta^{M} S_{0}^{*}=\pi E\left[L \mid M_{0}\right] E\left[X_{0}^{C B} \mid M_{0}\right]$ is given by

$$
\Delta^{M} S_{0}^{*}=\frac{\pi E\left[L \mid M_{0}\right] E\left[\bar{S} \mid M_{0}\right]}{2 \pi E\left[L \mid M_{0}\right]+\lambda R}-\frac{1}{2}\left\{S_{0}^{*, l=0}\right\}+\frac{\lambda R}{4 \pi E\left[L \mid M_{0}\right]+2 \lambda R}\left\{\frac{E\left[f \mid M_{0}\right]}{R}\right\} .
$$

What is the intuition behind this result? Because of Eq. (13), it is easy to show that the informed CB's optimal demand schedule at each point in time $t$ is now given by $X_{t}^{C B}=$ $\gamma\left[\bar{S}-\left(\frac{E\left[f \mid I_{\mathrm{t}}\right]}{R}-\pi X^{*}\right)+\frac{\lambda}{2 \pi L}\left(f-R S_{t}^{C B}\right)\right]$. The policy component of the intervention depends on $E\left[f \mid I_{t}\right]$ because so do both the investors' order flow $X_{i, t}$ and the expected transaction price $S_{t}^{*, l=0}$. Vice versa, the wealth-preservation motive is driven by the CB's knowledge of $f$. As we already emphasized in Section 3.1, the monopolist dealer, aware of the CB's intervention mechanism, uses both $E\left[f \mid I_{0}\right]$ and $E\left[f \mid M_{0}\right]$ to compute $E\left[X_{0}^{C B} \mid M_{0}\right]$. Therefore, he increases (decreases) his benchmark quotes if there is a positive probability $\left(E\left[L \mid M_{0}\right]>0\right)$ that the CB will buy (sell) GBP according to Eq. (39). Relaxing the full information assumption does not affect the absolute spread, because the MM, aware of whether his counterparty (when it arrives) is an investor or the (potentially informed) CB, does not experience any adverse selection risk, hence is

\footnotetext{
${ }^{30} \mathrm{As}$ in the full information framework, in both circumstances we impose similar sets of conditions to the parameters on the model (and to agents' beliefs) in order to interpret the wedge $S_{2, t}-S_{1, t}$ as the difference between the prices at which MMs are willing to sell and to buy GBP-denominated bonds.

${ }^{31}$ See the survey article by Sarno and Taylor (2001) for an analysis of the issue of secrecy usually permeating government activity in currency markets.
} 
not compensated for it while providing liquidity to the market, as it is instead the case in Naranjo and Nimalendran (2000). However, the proportional spread $P S_{0}$ does decrease (increases) if $E\left[X_{0}^{C B} \mid M_{0}\right]$ is positive (negative). Indeed, the MM can clear the market just by passing part of the extra potential revenues (costs) resulting from a positive (negative) intervention. Therefore, he revises $P S_{0}$ downward (upward), i.e., in the opposite direction of the quotes.

In stage 1 the first investors' order arrives, for example at time $t_{1}$. The reservation bid and ask prices at which the MM is willing to transact $X_{1, t_{1}}$ and $X_{2, t_{1}}, S_{1, t_{1}}$ and $S_{2, t_{1}}$, respectively, already account for his ensuing new belief that the CB is less likely to intervene in the future, $p_{l, t_{1}}$, where

$$
p_{l, t_{1}}=E\left[l \mid M_{0}, X_{i, t_{1}}\right]=E\left[l \mid M_{0}\right]-\frac{\operatorname{Var}\left[l \mid M_{0}\right]}{1-E\left[l \mid M_{0}\right]}<q_{l, t_{1}} .
$$

The difference between the quote revision at stage 0 and the one at stage 1 depends on the dispersion of the dealer's beliefs about $l$, that is, on the credibility of the CBs' announcement about the future intervention. If this credibility is lower, i.e., if the dispersion $\operatorname{Var}\left[l \mid M_{0}\right]$ is higher for a given $q_{l, t_{1}}=p_{l, 0}=E\left[l \mid M_{0}\right], E\left[L \mid M_{0}, X_{i, t_{1}}\right]$ is lower, the expected magnitude of the $\mathrm{CB}$ order is higher (as we have seen in Section 2.4), and so is the resulting quote revision with respect to the benchmark case, for $\frac{\partial \Delta^{\mathrm{M}} S_{0}^{*}}{\partial L}<0$. Consequently, investors' order flow induces more volatility in the transaction price, via the process of belief updating for $q_{l, t_{n}}$, when the monetary authority is less credible.

In stage 2, when the CB eventually intervenes (for example at time $t_{2}$ ), the dealer discounts his resulting new posteriors on $\bar{S}, f$, and $l$ into the price at which the transaction with the government occurs,

$$
\begin{aligned}
S_{t_{2}}^{C B}= & \frac{1}{R}\left\{E\left[\omega_{1 A} \mid M_{t_{1}}, X_{t_{2}}^{C B}\right] E\left[f \mid M_{0}, X_{t_{2}}^{C B}\right]\right\}+ \\
& +E\left[\omega_{1 B} \mid M_{t_{1}}, X_{t_{2}}^{C B}\right] \frac{E\left[f \mid I_{0}\right]}{R}+E\left[\omega_{2} \mid M_{t_{1}}, X_{t_{2}}^{C B}\right] \bar{S}+E\left[\omega_{3} \mid M_{t_{1}}, X_{t_{2}}^{C B}\right] \pi X^{*},
\end{aligned}
$$

where $E\left[\omega_{1 A} \mid M_{t_{1}}, X_{t_{2}}^{C B}\right]=\frac{\lambda R+\pi E\left[L \mid M_{\mathrm{t}_{1}}, X_{\mathrm{t}_{2}}^{\mathrm{CB}}\right]}{2 \pi E\left[L \mid M_{\mathrm{t}_{1}}, X_{\mathrm{t}_{2}}^{\mathrm{CB}}\right]+\lambda R}>0, E\left[\omega_{1 B} \mid M_{t_{1}}, X_{t_{2}}^{C B}\right]=-E\left[\omega_{3} \mid M_{t_{1}}, X_{t_{2}}^{C B}\right]=$ $-\frac{\pi E\left[L \mid M_{\mathrm{t}_{1}}, X_{\mathrm{t}_{2}}^{\mathrm{CB}}\right]}{\lambda R}<0$, and $E\left[\omega_{2} \mid M_{t_{1}}, X_{t_{2}}^{C B}\right]=\frac{2 \pi E\left[L \mid M_{\mathrm{t}_{1}}, X_{\mathrm{t}_{2}}^{\mathrm{CB}}\right]\left(\lambda R+\pi E\left[L \mid M_{\mathrm{t}_{1}}, X_{\mathrm{t}_{2}}^{\mathrm{CB}}\right]\right)}{\lambda R\left(2 \pi E\left[L \mid M_{\mathrm{t}_{1}}, X_{\mathrm{t}_{2}}^{\mathrm{CB}}\right]+\lambda R\right)}>0.32$ As in Proposition $3, S_{t_{2}}^{C B}$ is a weighted average of the MM's and investors' expectations about $f$, the revealed target level $\bar{S}$, and the risk-premium component $\pi X^{*} . E\left[f \mid I_{0}\right]$ has a negative weight in Eq. (41) because if investors are more pessimistic about the domestic currency, ceteris paribus the CB is expected to need a smaller positive $X_{t}^{C B}$ to chase the trend, or a bigger negative $X_{t}^{C B}$ to lean against the wind. Vice versa, $E\left[f \mid M_{t_{2}}\right]$ has a positive weight in $S_{t_{2}}^{C B}$ because if the NFV of buying GBP is greater, the $\mathrm{CB}$ is then expected to execute a bigger positive $X_{t}^{C B}$, or a smaller negative $X_{t}^{C B}$. In both cases, the dealer's optimal response to clear the market would then be to bid/offer less for one unit of GBP. $S_{t_{2}}^{C B}$ increases for higher $E\left[f \mid M_{0}, X_{t_{2}}^{C B}\right]$ because in that case the speculative component of CB total demand for GBP is expected to be bigger. In transacting

\footnotetext{
${ }^{32}$ Therefore, the price at which the trade with the CB is settled is regret-free in the sense of Glosten and Milgrom (1985), as it eventually depends on $X_{t_{2}}^{C B}$. Because we assume that investors' optimal demand schedules $X_{i, t_{\mathrm{n}}}$ are fully anticipated by the MMs, the prices at which their orders are cleared, $S_{i, t_{\mathrm{n}}}$, are regret-free as well.
} 
with the monetary authority, the MM uses sign and magnitude of the intervention to update his beliefs about $f$ and/or $\bar{S}$ (if $X_{t_{2}}^{C B}$ is unexpected) and his posterior $p_{l, t_{2}}=E\left[l \mid M_{t_{1}}, X_{t_{2}}^{C B}\right]$ (if $X_{t_{2}}^{C B}$ is unannounced) according to Proposition 6. In particular, $p_{l, t_{2}}$ becomes the MM's best prior for $l$ for the next trading rounds.

At this point the investors simply know that an intervention happened, but did not observe $X_{t_{2}}^{C B}$. Therefore, they cannot revise their beliefs about $f$ until a new transaction with an investor is executed. Indeed, the dealer embeds all his new information about $f, \bar{S}$, and $l$, in his potential prices for the next incoming investor of type $i$. When this investor arrives at stage 3 (for example at time $t_{3}$ ), that transaction is settled either at the new bid $S_{1, t_{3}}=S_{1,0}^{l=0}+\Delta^{M} S_{t_{3}}^{*}$ or at the new ask price $S_{2, t_{3}}=S_{2,0}^{l=0}+\Delta^{M} S_{t_{3}}^{*}$, where $\Delta^{M} S_{t_{3}}^{*}$ is given by

$$
\Delta^{M} S_{t_{3}}^{*}=\frac{\pi E\left[L \mid M_{t_{2}}, X_{i, \mathrm{t}_{3}}\right] \bar{S}}{2 \pi E\left[L \mid M_{t_{2}}, X_{i, \mathrm{t}_{3}}\right]+\lambda R}-\frac{1}{2}\left\{S_{0}^{*, l=0}\right\}+\frac{\lambda R}{4 \pi E\left[L \mid M_{t_{2}}, X_{i, \mathrm{t}_{3}}\right]+2 \lambda R}\left\{\frac{E\left[f \mid M_{t_{2}}\right]}{R}\right\} .
$$

The change in ${ }^{M} S_{t_{3}}^{*}$ with respect to $S_{0}^{*, l=0}\left(\Delta^{M} S_{t_{3}}^{*}\right)$ is due to both the imperfect substitutability $\left(p_{l, t_{3}}>0\right)$ and the signaling $\left(E\left[f \mid M_{t_{2}}\right] \neq E\left[f \mid M_{0}\right]\right)$ effects of the intervention. In fact, the MM used that transaction to learn about $f$ and $\bar{S}$, and then incorporated this new information in his new potential bid and ask quotes. The investors are aware of this process, and revise their beliefs about $p_{f}$ based on the sign of the difference between $S_{i, t_{3}}$ and the latest price $S_{i, t_{2-1}}$ registered before the CB actually intervened, along the lines of Proposition 7, i.e., $E\left[f \mid I_{0}, S_{i, t_{3}}-S_{i, t_{2-\mathrm{j}}}\right]={ }^{I} p_{f, t_{3}} f_{H}+\left(1-{ }^{I} p_{f, t_{3}}\right) f_{L}$.

This new expectation enters type 1 and type 2 investors' demand functions for domestic and GBP-denominated bonds, and is discounted into the quotes set by the MM for the next incoming order. Any future order flow, at time $t_{n}>t_{3}$, is then transacted at rates that reflect not only the dealer's and the investors' new beliefs about $f$ and $\bar{S}$ following the intervention but also the update to the dealer's prior about $l, q_{l, t_{n}}$ induced by the next arrivals, so that ${ }^{M} S_{t_{\mathrm{n}}}^{*}={ }^{M} S_{t_{3}}^{*}+\pi E\left[L \mid M_{t_{\mathrm{n}}}\right] E\left[X_{t_{\mathrm{n}}}^{C B} \mid M_{t_{\mathrm{n}}}\right]=S_{0}^{*, l=0}+\Delta^{M} S_{t_{\mathrm{n}}}^{*}$, where

$$
\begin{aligned}
\Delta^{M} S_{t_{\mathrm{n}}}^{*}= & \frac{\pi E\left[L \mid M_{t_{\mathrm{n}}}, X_{i, t_{\mathrm{n}}}\right] \bar{S}}{2 \pi E\left[L \mid M_{t_{\mathrm{n}}}, X_{i, t_{\mathrm{n}}}\right]+\lambda R}+\frac{1}{2}\left\{\frac{E\left[f \mid I_{t_{3}}\right]}{R}-\pi X^{*}\right\}+ \\
& +\frac{\lambda R}{4 \pi E\left[L \mid M_{t_{\mathrm{n}}}, X_{i, t_{\mathrm{n}}}\right]+2 \lambda R}\left\{\frac{E\left[f \mid M_{t_{2}}\right]}{R}\right\}-\left\{\frac{E\left[f \mid I_{t_{0}}\right]}{R}-\pi X^{*}\right\}
\end{aligned}
$$

and $E\left[L \mid M_{t_{\mathrm{n}}}, X_{i, t_{\mathrm{n}}}\right]=E\left[L \mid M_{t_{2}}, X_{i, t_{3}}, \ldots, X_{i, t_{\mathrm{n}}}\right]$. If the expected probability of future intervention $q_{l, t_{\mathrm{n}}}$ drops to zero after an intense flow of investors' orders, the reservation quotes $S_{i, t_{\mathrm{n}}}$ and the expected transaction price ${ }^{M} S_{t_{\mathrm{n}}}^{*}$ revert toward the pre-intervention levels $S_{i, 0}^{l=0}$ and $S_{0}^{*, l=0}$, respectively. The portfolio balance effect on the exchange rate is in fact smaller on the incoming risk-averse traders. However, the reversion is less than complete, as at the limit, for a $t_{N} \leq T$ such that $q_{l, t_{\mathrm{N}}}=0,{ }^{M} S_{t_{\mathrm{n}}}^{*}$ converges to:

$$
{ }^{M} S_{t_{\mathrm{N}}}^{*}=\frac{E\left[f \mid I_{t_{3}}\right]}{R}-\pi X^{*} \neq S_{0}^{*, l=0}
$$

The long-lived difference between ${ }^{M} S_{t_{\mathrm{N}}}^{*}$ and $S_{0}^{*, l=0}$ depends on the difference between $E\left[f \mid I_{t_{3}}\right]$ and $E\left[f \mid I_{0}\right]$, and is due exclusively to the fact that $\mathrm{CB}$ intervention, potentially informative 
about $f$ with probability $v$, induces a permanent revision in the investors' beliefs. Indeed, we can think of a typical cycle of CB actions starting with a public announcement such that initially $q_{l, 0}>0$, continuing with a trade $X_{t}^{C B}$ with the dealer(s), and ending with the market at large not expecting any additional intervention in the short-term, i.e., $q_{l, t_{\mathrm{N}}}=0$. Announcing a future intervention has an impact on quotes and spreads, but the sign and the magnitude of such impact depend crucially on the dealers' perceptions about the motives of the incoming CB trades $\left(E\left[f \mid M_{0}\right]\right.$ and $\left.E\left[\bar{S} \mid M_{0}\right]\right)$, on the credibility of the announcement $\left(\operatorname{Var}\left[l \mid M_{0}\right]\right)$, and on the investors' elasticity of demand for the risky GBP-denominated bonds $(\pi)$.. Because our stylized forex market is semi-strong efficient, the actual intervention is effective as long as it is unexpected and/or unannounced, i.e., just if it induces dealers and investors to revise their initial beliefs. Eventually, unless the monetary authority keeps the threat of intervention alive, or unless investors' expectations about $f$ have not been altered permanently by the previous intervention (as in Eq. (44)), the exchange rate eventually reverts to the levels at which the currency was traded at time zero.

To examine more carefully some of the implications of Eqs. (37) to (44) and to better understand the dynamics of the resulting intraday process of price formation, we simulate the sequence of events described in this section for a specific parametrization of the economy. We assume that 30 trades arrive during the interval $[0, T]$, and that for simplicity the same time elapses between each trade. We also assume (without loss of generality) that, consistently with some anecdotal and empirical evidence (e.g., Fischer and Zurlinden (1999) and Payne and Vitale (2001)), the CB order arrives in the morning, at time $t_{6}$. However, the dealer does not know with certainty when and if that order will ultimately come. We choose the true likelihood of intervention to be equal to 0.105, and then let the initial dealer's prior about $l, f_{M_{0}}(l)$, be a $\operatorname{Bet} a(a, b)$ with $a=266$ and $b=1215$, implying $q_{l, 0}=\frac{a}{a+b}=0.18$ (i.e., not centered on the true $l$ ) and $\operatorname{Var}\left[l \mid M_{0}\right]=\frac{a b}{(a+b+1)(a+b)^{2}}=0.0001 .^{33}$ As we did in Section 3, we select the initial endowments $\bar{X}_{1}$ and $\bar{X}_{2}$ and the variable $q$ so that type 1 and type 2 investors will be net sellers and net buyers, respectively, for any $t_{n} \in[0, T]$. We finally impose a parameter $\psi=0.30<\frac{1}{2}$, hence that the true correlation between $f$ and $\bar{S}$ is negative. A low $\psi$ implies that the informed $\mathrm{CB}$ is likely to try to fight the fundamental trend in the exchange rate behavior by setting low (high) target levels for high (low) true $f$, as it is typically the case for G7 currencies (e.g., Sarno and Taylor (2001)).

We start analyzing the case in which $f_{L}=\$ 1.45$ and $\bar{S}_{L}=\$ 1.31$ and the informed CB attempts to lean against the wind with a big negative order ${ }_{B} X_{t_{6}}^{C B}<0$ in Figure $5 \mathrm{a}$. The initial benchmark exchange rate, calculated according to Proposition 1 , is $S_{0}^{*, l=0}=\$ 1.4345$. We assume that the $\mathrm{CB}$ announcement at stage 0 is successful in pushing down $S_{1,0}^{l=0}$ and $S_{2,0}^{l=0}$ because, for $\psi<\frac{1}{2}$, the resulting $E\left[X_{t_{1}}^{C B} \mid M_{0}\right]$ is small and negative, although investors are pessimistic about the domestic currency $\left({ }^{I} q_{f, 0}=0.75\right)$ and the probability that the CB may be uninformed about $f$ is positive $((1-v)=0.2)$. Therefore, when it arrives, ${ }_{B} X_{t_{6}}^{C B}$ is unexpectedly big. The proportional spread $P S_{0}$ increases, as the dealer needs to pass some of the expected cash outflows from a future potential CB trade onto the population of investors in order to avoid to impose any drift on his inventory while simultaneously making some profits from trading. Our CB is also

\footnotetext{
${ }^{33}$ We choose the Beta distribution for computational ease, as the posterior distribution computed using Bayes' Rule is a Beta as well. The results that follow nonetheless hold for any other initial prior distribution for $l$ with support on $[0,1]$.
} 
not fully credible, for $\operatorname{Var}\left[l \mid M_{0}\right]>0$. Hence, the next few investors' orders, from $t_{1}$ to $t_{5}$, lowers the posterior likelihood of a future intervention to occur, inducing ${ }^{M} S_{t_{n}}^{*}$ to slightly increase (and the proportional spread to slightly decrease).

When the big and negative CB order arrives at $t_{6}$, such intervention (as shown in Proposition 6 ) is fully revealing to the dealer of the true $f$ and $\bar{S}$. The MM embeds this knowledge in his reservation quotes for the next incoming trades. Hence, ${ }^{M} S_{t_{7}}^{*}$ drops to around $\$ 1.3807$. When this occurs, investors observe the resulting negative $\Delta S_{i, t_{7}}$, hence infer that the CB was leaning against the wind. Consequently, they become more optimistic about the domestic currency $\left(E\left[f \mid I_{t_{7}}\right]<E\left[f \mid I_{0}\right]\right)$ and incorporate this new posterior in their demand function. So does the $\mathrm{MM}$ as well in pricing all future incoming orders. An additional downward (upward) adjustment of the quotes $S_{1, t_{8}}$ and $S_{2, t_{8}}$ (the proportional spread $P S_{t_{8}}$ ) ensues, due to the signaling content of the CB trade for the population of investors; its magnitude depends on $\pi$, or more precisely on those investors' demand elasticity, as illustrated by Eq. (44).

In stage 4 there are no more informative trades arriving to the market. Nonetheless, the investors' order flow from $t_{9}$ to $t_{30}$ drives down the dealer's belief about $l\left(q_{l, t_{n}}\right)$, according to Proposition 6, and its conditional variance $\operatorname{Var}\left[l \mid M_{t_{\mathrm{n}}}\right]=E\left[l^{2} \mid M_{t_{\mathrm{n}}}\right]-q_{l, t_{\mathrm{n}}}^{2}$, and drives up the absolute size of his expectation for a potential future intervention $\left(E\left[X_{t_{n}}^{C B} \mid M_{t_{n}}\right]\right)$. Hence, the threat of a future CB trade becomes less credible. Because the CB's optimal demand schedule is not a linear function of $L$, the effect of the resulting greater magnitude of $E\left[X_{t_{\mathrm{n}}}^{C B} \mid M_{t_{\mathrm{n}}}\right]$ $\left(\frac{\partial X_{t}^{\mathrm{CB}}}{\partial l}<0\right.$ in Section 2.4) on the market-clearing condition $E\left[Z_{t_{n}} \mid M_{t_{n}}\right]$ is more than outweighed by the effect of a lower and lower perceived likelihood of intervention. Consequently, the impact of imperfect substitutability on the quotes is weaker and weaker, the expected transaction price starts rising, and the proportional spread falls at an increasing rate. If we assume that eventually both $q_{l, t_{\mathrm{n}}}$ and $\operatorname{Var}\left[l \mid M_{t_{\mathrm{n}}}\right]$ drop to zero by time $t_{30}=T$, so does the portfolio balance effect on the reservation prices. Thus, the expected transaction price ${ }^{M} S_{t_{\mathrm{n}}}^{*}$ converges toward $S_{0}^{*, l=0}$, but does not return to its pre-announcement level. Indeed, ${ }^{M} S_{T}^{*}=\$ 1.3743<S_{0}^{*, l=0}$, i.e., closer than it was at $t=0$ to the target $\bar{S}_{L}=\$ 1.31$. We also find that $P S_{T}>P S_{0}^{l=0}$. Both $\Delta^{M} S_{T}^{*}<0$ and $\Delta P S_{T}>0$ stem from the signaling effect of $X_{t_{6}}^{C B}\left(E\left[f \mid I_{T}\right]<E\left[f \mid I_{0}\right]\right)$ : at time $T$ investors are more optimistic about the dollar than they were at stage 0 , hence relatively more willing to sell GBP; therefore, the monopolist MM reduces his bid and ask prices to clear the market, but not the absolute spread, in order to maximize at the same time his expected revenues from trading.

What happens to quotes and spreads if the monetary authority intervenes with an unexpectedly small negative order, i.e., when both $q_{f, 0}$ and $q_{\bar{S}, 0}$ are low but ${ }_{S} X_{t_{6}}^{C B}<0$ occurs instead? For the informed CB ${ }_{S} X_{t_{6}}^{C B}<0$ is optimal when $\bar{S}=\bar{S}_{L}$ but $f=f_{H}$, because of the ensuing trade-off between policy and wealth-preserving motives. Because there is a positive probability that the surprisingly small order came instead from an uninformed CB (i.e., that $f$ could be equal to $f_{L}$ ), beliefs' updates following its arrival are less than complete, along the lines of Proposition 6 . In particular, the new dealer's posterior $p_{f, t_{6}}$ is greater than $q_{f, 0}$ but lower than 1 . The resulting price update $\Delta^{M} S_{t_{7}}^{*}$ of stage 3 , albeit negative, may then be smaller than $\Delta^{M} S_{t_{5}}^{*}$. Therefore, the transaction prices at which orders $X_{i, t_{7}}$ are settled, $S_{i, t_{7}}=S_{i, 0}^{l=0}+\Delta^{M} S_{t_{7}}^{*}$, may increase (and the proportional spread may decrease) from the pre-intervention levels, even though the CB actually sold GBP, i.e., even if $\bar{S}=\bar{S}_{L}$. If at stage 4 the investors, unaware that ${ }_{S} X_{t_{6}}^{C B}<0$, observe a $S_{i, t_{7}}-S_{i, t_{7-\mathrm{j}}}>0$, they revise upward their (already high) beliefs about $p_{f}$, according to Proposition 7 , the $E\left[f \mid I_{0}, S_{i, t_{7}}-S_{i, t_{7-\mathrm{j}}}\right]$ that follows, higher (and not lower) than the original $E\left[f \mid I_{0}\right]$, 
is embedded in their demand schedules and in the dealer's reservation rates. Thus, the expected transaction price, ${ }^{M} S_{t_{8}}^{*}$, rises again and converges to levels higher than $S_{0}^{*, l=0}$ for $E\left[l \mid M_{T}\right] \longrightarrow 0$.

Thus, unexpectedly small interventions may generate undesired (or perverse) short- and longlived effects on quotes and spreads. The existence of such perverse, i.e., not unidirectional effects of $\mathrm{CB}$ interventions on the target exchange rate has been documented in the empirical literature. For example, Dominguez and Frankel (1993 a) report that purchases of dollars by the Fed during the period following the Louvre Accord in 1987 were consistently accompanied by a depreciation of the domestic currency in a market environment where investors were becoming more pessimistic about the long-term perspectives of the U.S. currency. Additionally, in the above scenario an unexpectedly small negative intervention may induce the proportional spread to fall, and not to increase, as it was instead the case for the arrival of an unexpectedly big negative order. These results suggest that not only the sign but also the magnitude of both the intervention and market's expectations about it play a crucial role in explaining its impact on the process of price formation in a currency market dominated by a monopolist dealer.

\subsection{The competitive dealers}

We now consider the circumstance in which dealers do not hold any market power, hence are unable to extract expected positive instantaneous rents from trading. We have shown in Sections 2.3 and 3.2 that, under the assumption of no inventory control, order processing costs, and full information, no bid-ask spread arises in our stylized forex market when $l=0$. The resulting expected exchange rate $S_{t}^{*, l=0}=\frac{f}{R}-\pi X^{*}$ corresponds to the price that would emerge if all risk-averse investors arrived at the same time in a competitive market. When instead $l>0$, we found that a wedge may be needed for the dealers to pass all additional revenues or costs from a potential CB order onto the population of investors, while at the same time still clearing the market.

In this subsection, we explore the impact of relaxing the full information assumption on these results. More specifically, using the previously described sequence of intraday events, we derive at each point in time $t_{n} \in[0, T]$ explicit solutions for the problem of competitive MMs (i.e., the equilibrium $S_{1, t_{n}}$ and $S_{2, t_{n}}$ such that $E\left[Z_{t_{n}} \mid M_{t_{n}}\right]=0$ and $E\left[\Pi_{t_{n}} \mid M_{t_{n}}\right]=0$ ) when their expectations $E\left[f \mid M_{t_{\mathrm{n}}}\right], E\left[S \mid M_{t_{\mathrm{n}}}\right]$, and $E\left[l \mid M_{t_{\mathrm{n}}}\right]$ replace $f, \bar{S}$, and $l$ and the investors' optimal demand (Eq. (3)) depends on $E\left[f \mid I_{t_{n}}\right]$ (instead of $f$ ). To do so, we further assume that the price set by the MMs for any transaction with the CB is the price that in full information would imply a zero spread, i.e., $S_{t_{\mathrm{n}}}^{C B}=S^{C B}(*)$ of Eq. (24). This hypothesis allows us to impose a zero spread in the benchmark full information scenario with $l>0$ of Proposition 4 by satisfying the binding no-instantaneous profit condition under market clearing. At each stage of the trading day (from 1 to 5), following a CB announcement at stage 0, the MMs update their beliefs about $f, \bar{S}$, and $l\left(E\left[f \mid M_{t_{\mathrm{n}}}\right], E\left[\bar{S} \mid M_{t_{\mathrm{n}}}\right], E\left[l \mid M_{t_{\mathrm{n}}}\right]\right.$, respectively), originally formulated at $t=0$, in the same way the monopolist MM does in Section 5.1, and consequently revise their potential prices with respect to the benchmark case of Proposition $1(l=0)$, so that

$$
S_{1, t_{\mathrm{n}}}=S_{1,0}^{l=0}+\pi E\left[L \mid M_{t_{\mathrm{n}}}\right] E\left[X_{1}^{C B} \mid M_{t_{\mathrm{n}}}\right]-\left\{(1-q) \frac{\pi}{2}\left(\bar{X}_{1}-\bar{X}_{2}\right)-\frac{\pi}{2} E\left[\Gamma \mid M_{t_{\mathrm{n}}}\right]\right\}
$$


and

$$
S_{2, t_{\mathrm{n}}}=S_{2,0}^{l=0}+\pi E\left[L \mid M_{t_{\mathrm{n}}}\right] E\left[X_{1}^{C B} \mid M_{t_{\mathrm{n}}}\right]+\left\{\frac{\pi q}{2}\left(\bar{X}_{1}-\bar{X}_{2}\right)-\frac{\pi q}{2(1-q)} E\left[\Gamma \mid M_{t_{\mathrm{n}}}\right]\right\},
$$

where $E\left[\Gamma \mid M_{t_{n}}\right]$ is obtained by replacing $A, C$, and $X_{t_{n}}^{C B}$ in $\Gamma$ (Proposition 4) with their expectations conditional on the information set $M_{0}, E\left[A \mid M_{t_{\mathrm{n}}}\right]=S_{0}^{*, l=0}+\pi E\left[L \mid M_{t_{\mathrm{n}}}\right] E\left[X_{t_{\mathrm{n}}}^{C B} \mid M_{t_{\mathrm{n}}}\right]$, $E\left[C \mid M_{t_{\mathrm{n}}}\right]=E\left[A \mid M_{t_{\mathrm{n}}}\right]\left(\frac{E\left[A \mid M_{\mathrm{t}_{\mathrm{n}}}\right]}{\pi q}+\bar{X}_{1}-\frac{R}{\pi} E\left[f \mid I_{t_{\mathrm{n}}}\right]\right)-E\left[L \mid M_{t_{\mathrm{n}}}\right] E\left[X_{t_{\mathrm{n}}}^{C B} \mid M_{t_{\mathrm{n}}}\right] S^{C B}(*)$, and

$$
\begin{aligned}
E\left[X_{t_{\mathrm{n}}}^{C B} \mid M_{t_{\mathrm{n}}}\right]= & \frac{1}{\pi E\left[L \mid M_{t_{\mathrm{n}}}\right]}\left\{E\left[\bar{S} \mid M_{t_{\mathrm{n}}}\right]-\left(\frac{E\left[f \mid I_{t_{\mathrm{n}}}\right]}{R}-\pi X^{*}\right)\right\}+ \\
& +\frac{\lambda}{2\left(\pi E\left[L \mid M_{t_{\mathrm{n}}}\right]\right)^{2}}\left\{E\left[f \mid M_{t_{\mathrm{n}}}\right]-R S^{C B}(*)\right\},
\end{aligned}
$$

respectively. Therefore, information asymmetry induces the following wedge between equilibrium offer and bid quotes:

$$
S_{2, t_{\mathrm{n}}}-S_{1, t_{\mathrm{n}}}=\frac{\pi}{2}\left(\bar{X}_{1}-\bar{X}_{2}\right)-\frac{\pi}{2(1-q)} E\left[\Gamma \mid M_{t_{\mathrm{n}}}\right]
$$

Such wedge is generally different from zero, hence different from the benchmark scenario of Proposition $1(l=0)$ and from the full information scenario of Section $3.2\left(l>0\right.$ and $S_{t}^{C B}=$ $\left.S^{C B}(*)\right)$. It is also different from the absolute spread quoted in the monopolistic scenario of the previous subsection, $\frac{\pi}{2}\left(\bar{X}_{1}-\bar{X}_{2}\right)$. When $E\left[l \mid M_{t_{n}}\right]>0$, competitive MMs must dissipate all the additional expected costs or revenues from expected CB trades onto the population of investors while clearing the market. Because of imperfect substitutability, this can be accomplished only by an asymmetric revision of reservation quotes, as in Proposition 4. The monopolist MM is instead able to retain part of those expected revenues, or to pass part of those expected costs to the risk-averse investors. When there is information asymmetry between dealers and the informed $\mathrm{CB}\left(E\left[\Gamma \mid M_{t_{\mathrm{n}}}\right] \neq \Gamma\right)$, this effect is enhanced by the uncertainty surrounding $l\left(\operatorname{Var}\left[l \mid M_{t_{\mathrm{n}}}\right]\right)$ and sign and magnitude of the expected intervention $\left(E\left[X_{t_{\mathrm{n}}}^{C B} \mid M_{t_{\mathrm{n}}}\right] \neq X_{t_{\mathrm{n}}}^{C B}\right)$ making more difficult for the competitive MMs to transfer the resulting expected cash flows to the risk-averse investors while maintaining their inventory driftless. Such uncertainty does not affect the spread set by the monopolist MM, for he still finds optimal to adjust his quotes symmetrically (as in the full information scenario) to maximize his expected profits from trading. Therefore, an equilibrium nonzero wedge arises in Eq. (48), although we imposed that $S_{t_{\mathrm{n}}}^{C B}=S^{C B}(*)$, hence that the full information competitive spread $S_{2, t_{\mathrm{n}}}-S_{1, t_{\mathrm{n}}}=0$ (Eq. (23)).

To facilitate the intuition for those bid and offer rates we simulate the case in which an informed CB arrives to the market at time $t_{6}$ with an unexpectedly big negative trade in the attempt to lean against the wind, using the same model parametrization adopted in Figure 5a, and display the resulting patterns for ${ }^{C} S_{t_{\mathrm{n}}}^{*}$ and $P S_{t_{\mathrm{n}}}$ in Figure 5b. This experiment also allows us to compare the impact of the same CB action on the intraday process of price formation in our stylized forex market for different degrees of market power. At the beginning of the day, the announcement by the monetary authority induces a small downward revision in quotes, as dealers expect the CB to sell a small amount of foreign currency, while both $S_{2,0}-S_{1,0}$ and $P S_{0}$ decline (i.e., become negative, for $S_{1,0}^{l=0}=S_{2,0}^{l=0}$ in Eq. (6)) as a result of $E\left[X_{0}^{C B} \mid M_{0}\right]<0$. 
Why is this occurring? To clear the market, competitive dealers need to attract more net buyers and less net sellers. To do so, they mark down both their bid and offer prices. Additionally, because those dealers expect a cash outflow from the potential CB trade, they need to generate positive expected net revenues from trading with the investors to satisfy the binding condition that $E\left[\Pi_{0} \mid M_{0}\right]=0$. For the given set of model's parameters, this is achieved by reducing the absolute and proportional spreads. When the MM holds significant market power instead (as in the example of Figure 5a), the no-expected drift condition is the only constraint to his actions. Therefore, he is still able to charge a positive spread and extract rents from the trading process.

Absolute and proportional spreads increase slightly toward the $l=0$ levels for the next few uninformative trades with investors, as $q_{l, t_{n}}$ declines along the lines of Proposition 6 , and so does $\operatorname{Var}\left[l \mid M_{t_{\mathrm{n}}}\right]$. When eventually the CB intervenes (at time $t_{6}$ ) with a big negative order, sign and magnitude of the trade are fully revealing of $\bar{S}_{L}$ and $f_{L}$. Quotes are consequently again revised downward, but both the absolute and proportional spreads now increase, this time as in the monopolistic scenario of Figure 5a, because at the new posteriors the MMs expect bigger future negative orders $X_{t_{\mathrm{n}}}^{C B}$. The new spread at $t_{7}$, in stage 3 , is very close to zero by construction, as now $E\left[X_{t_{7}}^{C B} \mid M_{t_{7}}\right]=X_{t_{7}}^{C B}$ and $S_{t_{7}}^{C B}=S^{C B}(*)$, but not exactly so because $E\left[f \mid I_{t_{7}}\right]>f_{L}$. Only afterwards (at $t_{8}$ ) do investors learn from the observed transaction prices that a negative intervention must have occurred, and become consequently more optimistic about the domestic currency. Hence, they demand less GBP, thus inducing a smaller expected future intervention and a consequent bigger increase of the proportional spread toward the initial levels when $l=0$.

For the rest of the trading day, no more informative orders hit the dealers' screens. Nonetheless, the MMs keep updating their priors about $l$ according to Eq. (40). The arrival of $X_{i, t_{n}}$ implies more uncertainty about whether the CB is going to arrive again, higher $\operatorname{Var}\left[l \mid M_{t_{\mathrm{n}}}\right]$, and a bigger expected absolute size of that potential future intervention. This uncertainty translates not only in higher transaction prices but also in smaller absolute and proportional spreads. More interestingly, the spreads decreases at an increasing rate, and at a faster pace than in the monopolistic case of the previous subsection. This implies, as evident from Figure 5b, that the time series of observed $\left(S_{i, t_{\mathrm{n}}}\right)$ and expected $\left({ }^{C} S_{t_{\mathrm{n}}}^{*}\right)$ transaction prices are more volatile. What is the intuition behind this result? At the beginning of the day, price volatility is high because the announcement of a future intervention increased the dispersion of beliefs among market participants. Indeed, the dealers, expecting a small negative CB order, revise downward their reservation quotes, although investors forecast a weaker dollar $\left({ }^{I} q_{f, 0}=0.75\right)$. When the intervention actually occurs and the information learned from it is conveyed to all market participants, then new beliefs are formed, the policy motives are fully revealed, and the exchange rate volatility subsides.

Lower $q_{l, t_{n}}$ resulting from investors' order flow and increasing uncertainty surrounding it make more difficult for the dealers to clear the market at each point in time, for the composition of the incoming demand for GBP is becoming more difficult to predict. Additionally, competitive MMs still have to pass all expected extra cash flows from a potential future CB trade onto the population of investors. Given the current demand elasticity and the fact that now investors, being more optimistic about the dollar, are less willing to buy the GBP-denominated assets that an incoming monetary authority would sell, bigger (but less likely) $E\left[X_{t_{n}}^{C B} \mid M_{t_{n}}\right]$ induces absolute and proportional spreads to decrease, and transaction prices (and price volatility) to increase. When $q_{l, t_{n}}$ eventually converges to zero (by time $T$ in Figure $5 \mathrm{~b}$ ), the conditional variance $\operatorname{Var}\left[l \mid M_{t_{n}}\right]$ declines sharply as well. Hence, the threat of a future intervention becomes 
less significant and credible, and affects less the process by which dealers formulate offer and bid quotes. Therefore, intraday transaction price volatility subsides, the absolute spread rises toward the initial pre-announcement level, and so does $P S_{t_{\mathrm{n}}}$ toward $P S_{T}=0=P S_{0}^{l=0}$.

Nonetheless, even without portfolio balance effects, eventually the expected transaction price at $T$ (assuming that $q_{l, t_{30}}=q_{l, T}=0$ ) is equal to ${ }^{M} S_{T}^{*}$ and is given by ${ }^{C} S_{T}^{*}=\$ 1.3743<S_{0}^{*, l=0}$. More generally, it is easy to see that Eq. (44) applies to the competitive dealership case as well for each possible $X_{t_{n}}^{C B}$. This result has two interesting normative implications for an active monetary authority. First, the CB trade has the same long-lived effect on quotes (but not on spreads) in both the monopolist and competitive scenarios of Figures 5a and 5b, respectively, if the threat of future intervention is not significant $\left(E\left[X_{T}^{C B} \mid M_{T}\right]=0\right)$ or not credible $\left(q_{l, T}=0\right)$ by the end of the interval $[0, T]$. That effect is due exclusively to the CB having signaled information about $f$ and $\bar{S}$ through its transactions (and to the investors' updating mechanism for their beliefs), consistently with the empirical findings of Pasquariello (2002). Portfolio balance effects of interventions on the exchange rate are instead short-lived. Indeed, if $E\left[f \mid I_{T}\right]=E\left[f \mid I_{0}\right]$ then ${ }^{C} S_{T}^{*}=S_{0}^{*, l=0}$ unless $q_{l, T}>0$. In our model this is due to the structure of currency trading, i.e., to the fact that trading occurs sequentially, and not to exogenous market integration increasing the substitutability of domestic and foreign currency-denominated assets (as suggested, for example, by Edison (1993) and Sarno and Taylor (2001)). This observation has so far been ignored, to our knowledge, by the financial and economic literature on exchange rates.

Second, with information asymmetry the intervention is more effective (in both the shortand long-term) than in the monopoly case as long as $E\left[l \mid M_{t_{n}}\right]>0$. In fact, for the same set of parameters and initial priors, immediately following investors' beliefs update in Figure 5b, ${ }^{C} S_{t_{8}}^{*}=\$ 1.3270$ is lower than ${ }^{M} S_{t_{8}}^{*}=\$ 1.3506$ (thus closer to $\bar{S}_{L}=\$ 1.31$ ), and remains so as long as $q_{l, t_{n}}>0$ because the portfolio balance effects of $E\left[X_{t_{n}}^{C B} \mid M_{t_{n}}\right] \neq 0$ on $S_{i, t_{n}}$ (i.e., the ensuing quotes' revisions $\Delta S_{i, t_{n}}$ ) are more significant when the MMs are unable to extract rents from the trading process, as we had already suggested in Section 3.3. However, if the perceived threat of a future intervention becomes increasingly less credible (if $q_{l, t_{n}}$ declines rapidly toward zero), then $S_{t_{n}}^{*}$ converges to $\frac{E\left[f \mid I_{T}\right]}{R}-\pi X^{*}$ of Eq. (44), regardless of the degree of market power held by the dealers. The unsuccessful attempts by the (generally less credible) monetary authorities of several Asian countries during 1997 and 1998 to lean against the wind and rescue their ailing exchange rates seem to offer some anecdotal support to these findings, for their domestic currency markets were characterized by a smaller number of dealers holding more market power.

\section{Conclusions}

In this paper we developed a theory of the impact of sterilized Central Bank intervention on the microstructure of currency markets, and showed that this impact is indeed significant and non-trivial. We analyzed the two major channels of effectiveness of such intervention, imperfect substitutability and signaling, in a model of sequential trading in which the stylized monetary authority is a rational, but not necessarily profit-maximizing player. In the basic framework of our model, domestic risk-averse traders do not reach the market simultaneously but according to an exogenously specified arrival process with their demand for risky foreign currency-denominated bonds. Prices are set by a monopolist, or by competitive risk-neutral dealers who maximize expected instantaneous net revenues from trading under a market-clearing constraint. In such 
framework, the rational CB's demand schedule results endogenously from the optimal resolution of a potential trade-off between policy and wealth-preservation motives. This trade-off makes interventions leaning against the wind more painful, or alternatively more expensive than if chasing the trend.

When all agents are perfectly informed about the parameters of the economy and CB's objectives, the market is strong-form efficient. Full information restricts the effectiveness of an intervention to the portfolio balance scenario in which risk-averse investors have to be compensated for having to hold more (or less) of the foreign currency than they otherwise would were the CB trade unlikely. Under the more realistic assumption that investors and dealers are not perfectly informed about fundamentals and CB's policy motives, the market is semi-strong efficient and actions by the monetary authority may signal information to those less informed agents. In both settings, we showed that the introduction of a CB has a significant impact on the process of intraday price formation in our stylized currency market. Dealers' reservation quotes are revised upward (downward) as soon as the CB is expected to buy (sell) the foreign currency with positive likelihood. The effect of CB trades on absolute and proportional spreads depends crucially on the degree of market power held by the market-makers. In the extreme scenario of a monopolist dealer, the absolute spread is unchanged by the intervention. However, the proportional spread increases (decreases) when the monetary authority is leaning against the wind (chasing the trend). This occurs because the dealer is attempting to retain (pass) at least some of the additional revenues (costs) resulting from the $\mathrm{CB}$ action in order to maximize his expected profits, while at the same time keeping his expected inventory driftless. When there are many dealers competing for the incoming trade, all potential cash inflows (outflows) resulting from expected positive (negative) government orders have to be transferred onto the population of investors. Because of imperfect substitutability of domestic and foreign currency-denominated assets, this can be achieved only by creating a wedge between bid and offer prices.

This intuition is critical as well to understand why in our model a CB attempting to push the exchange rate toward a target level is generally more successful when forex dealers hold less market power. A significant degree of market power hampers such effectiveness because the market-makers, in their quest for profit-maximization, do not adjust their quotes completely in response to the $\mathrm{CB}$ trade. When there is instead a multitude of dealers, costs and revenues from those CB actions are transmitted fully to the risk-averse investors, hence the resulting quotes' revisions are more substantial. We also demonstrated that interventions have a permanent effect on the exchange rate when the transacted CB order is informative, or when the threat of future government actions is significant and credible. In particular, we showed that portfolio balance effects are short-lived (consistently with available empirical evidence) because of sequential trading, without having to invoke increasing financial integration and substitutability between financial assets denominated in different currencies.

In our model, changes in transaction prices, in bid and offer quotes, and in the resulting absolute and proportional spreads are found to be related not only to the sign but also to the magnitude of the incoming CB order. Small CB trades are more easily accommodated by forex dealers and more easily absorbed by risk-averse investors. Small orders are also not fully revealing of the objectives and knowledge of an informed CB. Hence, they may induce less substantial or, if unexpected, even undesired (or perverse) updates of marketwide beliefs, and eventually smaller revisions in demands, transaction prices, and spreads. Furthermore, the speed of adjustment in 
those prices following a CB trade is shown to depend crucially on the degree of transparency of the order flow in the market, i.e., on the process by which uninformed investors update their beliefs over time. Finally, simulations of the intraday pattern of equilibrium transaction prices in our setting revealed that exchange rate volatility tends to be high before an intervention occurs, subsides when new information is conveyed to dealers and investors by the executed CB order, then resumes following increasing uncertainty surrounding future actions by the monetary authority, and eventually declines when a new intervention is perceived to be more unlikely.

This paper offers many avenues for future research. Several are theoretical. For example, our model focuses on the circumstance in which investors update their beliefs about the fundamental value of the currency just with a delay with respect to the intervention, because they cannot observe the amount transacted. This delay, we argued, slows down the adjustment of the exchange rate in the direction targeted by the monetary authority. However, we do not consider the possibility that just one of the competing market-makers receives the $\mathrm{CB}$ order and that the subsequent "hot potato" transmission of flows in the inter-dealer market, as in Lyons (1997) and Evans and Lyons (2001), may affect differently quotes and spreads. Allowing market-makers to trade with each other during the day is a challenging yet desirable extension of our basic framework, as Lyons (2001) reports that inter-dealer trading explains up to $75 \%$ of total daily volumes in the global currency markets. Other avenues are more properly empirical. Our model generates a rich set of implications of the positive likelihood of an incoming intervention and of its arrival for intraday spreads, price revisions, exchange rate returns, and return volatility. The recent availability of tick-by-tick transaction data for currency trades executed on behalf of monetary authorities, e.g., the database of Fischer and Zurlinden (1999), offers an unparalleled opportunity for financial economists to study in a comprehensive manner the process by which dealers buy and sell foreign currency over a typical trading day.

\section{Appendix}

Proof of Proposition 3. Given the problem in Eq. (15), it is straightforward to show that the equilibrium exchange rate quoted by the MM for a CB intervention is given by Eq. (18) with

$$
\begin{aligned}
\omega_{1} & =\frac{\lambda^{2} R^{2}-2 \pi^{2} L^{2}}{\lambda R(2 \pi L+\lambda R)}, \\
\omega_{2} & =\frac{2 \pi L(\pi L+\lambda R)}{\lambda R(2 \pi L+\lambda R)}>0, \\
\omega_{2} & =\frac{\pi L}{\lambda R}>0 .
\end{aligned}
$$

We assume that parameters in the model are such that $S_{t}^{C B}$ is always positive. Plugging $S_{t}^{C B}$ in $X_{t}^{C B}$ we have

$$
X_{t}^{C B}=\gamma\left[\bar{S}\left(1-\frac{\lambda R \omega_{2}}{2 \pi L}\right)-\frac{f}{R}\left(1+\frac{\lambda R \omega_{1}}{2 \pi L}-\frac{\lambda R}{2 \pi L}\right)+\pi X^{*}\left(1-\frac{\lambda R \omega_{3}}{2 \pi L}\right)\right],
$$


while plugging $X_{t}^{C B}$ into Eqs. (16) and (17) gives

$$
\begin{aligned}
S_{i, t} & =S_{i, t}^{l=0}+\frac{\pi L}{2 \pi L+\lambda R}\left(\bar{S}-\frac{f}{R}\right)+\frac{\pi}{2} X^{*}= \\
& =\bar{S}\left(\frac{\pi L}{2 \pi L+\lambda R}\right)+\frac{f}{R}\left(\frac{\pi L+\lambda R}{2 \pi L+\lambda R}\right)-\frac{\pi}{2} \bar{X}_{i} .
\end{aligned}
$$

We finally use those prices and Eq. (3) to find the optimal orders for the investors,

$$
X_{i, t}=P-\frac{1}{\pi}\left[S_{i, t}^{l=0}+\pi L X_{t}^{C B}\right]-\bar{X}_{i}
$$

Eq. (A-6) implies that $X_{i, t}=P-\frac{1}{\pi} S_{i, t}^{l=0}-\bar{X}_{i}-L X_{t}^{C B}$, and $X_{2, t}-X_{1, t}=\frac{\bar{X}_{1}-\bar{X}_{2}}{2}$. It is then clear that, as in Saar (2000a), $X_{1, t} \neq X_{2, t}$, thus satisfying the dealer's initial conjecture, unless $\bar{X}_{1}=\bar{X}_{2}$, i.e., unless we are in the economy where there would be no trading (and risk-sharing), and no CB. In that economy there would be a population of investors of just one type (for example, net buyers of foreign currency), and the market would open only when the MM expects that $X_{t}^{C B}<0$. Finally, we check that the CB's initial conjecture that $E\left[S_{t}^{*} \mid \mathrm{z}_{t}\right]$ be equal to $q S_{1, t}+(1-q) S_{2, t}$ is indeed correct in equilibrium. In fact, from Eq. (A-5) it follows that:

$$
q S_{1, t}+(1-q) S_{2, t}=\left(\frac{f}{R}-\pi X^{*}\right)+\left[\left(\bar{S}-\frac{f}{R}\right)\left(\frac{\pi L}{2 \pi L+\lambda R}\right)+\frac{\pi}{2} X^{*}\right]
$$

while, after some tedious substitutions, Eq. (A-4) implies that

$$
X_{t}^{C B}=\frac{1}{\pi L}\left[\left(\bar{S}-\frac{f}{R}\right)\left(\frac{\pi L}{2 \pi L+\lambda R}\right)+\frac{\pi}{2} X^{*}\right]
$$

It is then clear that $\left(\frac{f}{R}-\pi X^{*}\right)+\pi L X_{t}^{C B}=q S_{1, t}+(1-q) S_{2, t}$, as originally conjectured by our CB. Finally, it is easy to show that, for $l>0$, the resulting investors' optimal orders are ${ }^{M} X_{2, t}>0$ and ${ }^{M} X_{1, t}<0$ if $\bar{X}_{2}<2\left[\frac{f}{R}\left(\frac{\pi L}{2 \pi^{2} L+\lambda R}\right)-\bar{S}\left(\frac{L}{2 \pi L+\lambda R}\right)\right]<\bar{X}_{1}$, i.e., when $\bar{X}_{1}$ is "sufficiently" high and $\bar{X}_{2}$ is "sufficiently" low.

Proof of Proposition 4. The construction of the equilibrium involves the same three steps as in the proof of Proposition 3. However, in this case we use the market-clearing condition $E\left[Z_{t} \mid M_{t}\right]=0$ to express $S_{1, t}$ as a function of $S_{2, t}$ and $S_{t}^{C B}$ (Eq. (21)). Then, plugging the optimal demand schedules of investors and CB in the no-profit condition $E\left[\Pi_{t} \mid M_{t}\right]=0$ generates a quadratic equation with respect to $S_{2, t}$, whose solutions are given by

$$
S_{2, t}=\frac{f}{R}-\pi X^{*}+\pi L X_{t}^{C B}+\frac{\pi q}{2}\left(\bar{X}_{1}-\bar{X}_{2}\right) \pm\left[\frac{\pi q}{2(1-q)}(\Gamma)\right]
$$

where $\Gamma=\left\{(1-q)^{2}\left[\left(\bar{X}_{2}-\bar{X}_{1}\right)-\frac{2 A}{\pi q}\right]^{2}-4\left(\frac{1-q}{\pi q}\right) C\right\}^{\frac{1}{2}}>0, A=\bar{S}+\frac{\lambda}{2 \pi L}\left(f-R S_{t}^{C B}\right)$, and $C=A\left(\frac{A}{\pi q}+\bar{X}_{1}-\frac{R}{\pi} f\right)-L X_{t}^{C B} S_{t}^{C B}$. We choose, and report in Eq. (22), the expression for $S_{2, t}$ implied by the minus sign multiplying the squared term $\Gamma$, as it represents the only solution 
reverting to the case of Proposition 1 (Eq. (6)) when $l=0$ and $\bar{X}_{1}>\bar{X}_{2}$. Because of the definition of $\Gamma$, we need to impose that the parameters of the model are such that

$$
(1-q)^{2}\left[\left(\bar{X}_{2}-\bar{X}_{1}\right)-\frac{2 A}{\pi q}\right]^{2}-4\left(\frac{1-q}{\pi q}\right) C>0 .
$$

This condition is satisfied when $\left|\bar{X}_{1}-\bar{X}_{2}\right|$ is not "too" small. Finally, we verify that the dealers' initial conjectures are confirmed under the selected set of equilibrium prices. It easily follows from substituting Eqs. (21) and (22) into the investors' optimal demand functions of Eq. (3) that $X_{1, t} \neq X_{2, t}$ if and only if $\left(\bar{X}_{1}-\bar{X}_{2}\right) \neq-\frac{1}{(1-q)} \Gamma$. For the set of parameters such that the restriction of Eq. (A-10) applies, $\left(\bar{X}_{1}-\bar{X}_{2}\right)$ always differs from $-\frac{1}{(1-q)} \Gamma$ when $\bar{X}_{1}>\bar{X}_{2}$, as in our model. This implies from the fact that, for $\bar{X}_{1}>\bar{X}_{2}$, we have chosen the solution implied by the minus sign in Eq. (A-9). If instead $\bar{X}_{1}<\bar{X}_{2}$, then we would choose the solution implied by the plus sign in order to have $S_{1, t}$ and $S_{2, t}$ reverting to the case of Proposition 1 when $l=0$. In such a circumstance, it would then follow that $X_{1, t} \neq X_{2, t}$ if and only if $\left(\bar{X}_{2}-\bar{X}_{1}\right) \neq-\frac{1}{(1-q)} \Gamma$. But again this is always true, given the original assumption about the types' endowments $\left(\bar{X}_{1}<\bar{X}_{2}\right)$ and the fact that $\Gamma>0$. Hence, as in the monopoly case, dealers' initial conjecture about the investors' types is verified in equilibrium. To save space, we do not report here the last step of verifying that the CB's initial conjecture that $E\left[S_{t}^{*} \mid \mathbf{z}_{t}\right]$ be equal to $q S_{1, t}+(1-q) S_{2, t}$ is indeed correct in equilibrium. Now, we can search for the price $S_{t}^{C B}$ such that $S_{2, t}-S_{1, t}=0$. It is clear from Proposition 1 that this is the case if the CB is not intervening (i.e., if $l=0$ and/or $X_{t}^{C B}=0$ ). Hence, when $l>0$, it is possible to find the (extremely high or extremely low) price at which our monetary authority (willing to chase the trend or lean against the wind, respectively) does not find optimal to intervene, i.e., such that $X_{t}^{C B}=0$. Because in this study we focus on the impact of CB interventions on quotes and spreads, $S_{t}^{C B}(*)$ in the text will instead always be the one price charged by competitive dealers to the CB such that $S_{2, t}-S_{1, t}=0$ but $X_{t}^{C B} \neq 0$. In order to find this price, we first observe that $E\left[Z_{t} \mid M_{t}\right]=0$ implies that $q X_{1, t}+(1-q) X_{2, t}=-L X_{t}^{C B}$. Hence, for $E\left[\Pi_{t} \mid M_{t}\right]=0$ to hold at a zero spread and $X_{t}^{C B} \neq 0$, it has to be true that $S_{1, t}=S_{2, t}=S_{t}^{C B}$. Moreover, we have seen in Section 2.4 that plugging the optimal investors' demand for GBP-denominated bonds of Eq. (3) in $E\left[\Pi_{t} \mid M_{t}\right]=0$ gives

$$
q S_{1, t}+(1-q) S_{2, t}=S_{t}^{C B}=\frac{f}{R}-\pi X^{*}+\pi L X_{t}^{C B} S_{t}^{C B}
$$

Lastly, we substitute $X_{t}^{C B}$ in Eq. (A-11) with the optimal intervention schedule of Eq. (14), solve for $S_{t}^{C B}(*)$, and find

$$
S_{t}^{C B}(*)=S_{1, t}=S_{2, t}=\left(\frac{2 \pi L}{2 \pi L+\lambda R}\right) \bar{S}+\left(\frac{\lambda}{2 \pi L+\lambda R}\right) f .
$$

It is easy to verify that, when $S_{t}^{C B}=S_{t}^{C B}(*)$, investors and CB's conjectures are verified in equilibrium, i.e., that $X_{1, t} \neq X_{2, t}$ for $\bar{X}_{1} \neq \bar{X}_{2}$ (as then $\Gamma=0$ and $\left(\bar{X}_{1}-\bar{X}_{2}\right)=-\frac{1}{(1-q)} \Gamma$ for $\bar{X}_{1}=\bar{X}_{2}$ and $\left.S_{t}^{C B}=S_{t}^{C B}(*)\right)$, and that $E\left[S_{t}^{*} \mid \mathbf{z}_{t}\right]$ is equal to $q S_{1, t}+(1-q) S_{2, t}$. If $S_{t}^{C B}=$ $S_{t}^{C B}(*)$, then ${ }^{C} X_{2, t}=2{ }^{M} X_{2, t}$ and ${ }^{C} X_{1, t}=2{ }^{M} X_{1, t}$. Hence, to ensure that the resulting investors' 
optimal orders will be ${ }^{C} X_{2, t}>0$ and ${ }^{C} X_{1, t}<0$, the same restriction on the model's parameters reported in the proof of Proposition 3 applies. If instead $S_{t}^{C B} \neq S_{t}^{C B}(*)$, it can be shown that ${ }^{C} X_{2, t}>0$ and ${ }^{C} X_{1, t}<0$ when the following restrictions on the model's parameters (and the chosen $S_{t}^{C B}$ ) are satisfied:

$$
\begin{gathered}
\bar{X}_{2}+\frac{q}{2}\left(\bar{X}_{1}-\bar{X}_{2}\right)-\frac{q}{2(1-q)}(\Gamma)<\left[P-\frac{1}{\pi} \bar{S}-\frac{\lambda}{2 \pi^{2} L}\left(f-R S_{t}^{C B}\right)\right] \\
\bar{X}_{1}-\frac{(1-q)}{2}\left(\bar{X}_{1}-\bar{X}_{2}\right)+\frac{1}{2}(\Gamma)>\left[P-\frac{1}{\pi} \bar{S}-\frac{\lambda}{2 \pi^{2} L}\left(f-R S_{t}^{C B}\right)\right],
\end{gathered}
$$

i.e., again when $\bar{X}_{1}$ is "sufficiently" high and $\bar{X}_{1}$ is "sufficiently" low, as in Proposition 3. For $l=0$ these restrictions reduce to our original assumption that $\bar{X}_{1}>\bar{X}_{2}$.

Proof of Proposition 5. The first statement of Proposition 5 ensues from the marketclearing condition $E\left[Z_{t} \mid M_{t}\right]=0$ implying that $S_{t}^{*}=\bar{S}+\frac{\lambda}{2 \pi L}\left(f-R S_{t}^{C B}\right)$. If $\lambda=0$ in $L(\bar{S}, \lambda)$, then ${ }^{C} S_{t}^{*}=\bar{S}$ while ${ }^{M} S_{t}^{*}=\frac{1}{2} \bar{S}+\frac{1}{2}\left(\frac{f}{R}-\pi X^{*}\right)$. Clearly ${ }^{M} S_{t}^{*}=\bar{S}$ just when $\frac{f}{R}-\pi X^{*}=\bar{S}$, i.e., just when the intervention is actually not needed, hence does not occur. Finally, if we substitute Eqs. (25) and (26) into Eq. (27), it is easy to see that $E M_{t}<0$ if and only if $\left(f-R^{C} S_{t}^{C B}\right)^{2}>\frac{4 \pi^{2} L^{2}}{\lambda^{2}}\left[\left(\frac{f}{R}-\bar{S}\right)\left(\frac{\pi L+\lambda R}{2 \pi L+\lambda R}\right)-\frac{\pi}{2} X^{*}\right]^{2}$. For reasonable parametrizations of the model, this will happen just for "very small" or "very high" values of ${ }^{C} S_{t}^{C B}$ and/or for very high values of $\lambda$. In those circumstances, wealth-preservation is "too" important for the CB, that consequently resists the monopolist MM's attempts to maximize profits at its expenses. Additionally, it is possible to show that if $\lambda$ is "small" or if the NFV of investing in GBP-denominated bonds $\left(f-R S_{t}^{C B}\right)$ is positive when the CB is chasing the trend (but ${ }^{M} S_{t}^{C B}>{ }^{C} S_{t}^{C B}$ ) and negative when the CB is instead leaning against the wind (but ${ }^{M} S_{t}^{C B}<{ }^{C} S_{t}^{C B}$ ), Proposition 5 applies to $L^{M}(\bar{S}, \lambda)$ versus $L^{C}(\bar{S}, \lambda)$ as well. In fact, their difference is given by $E M_{t}+\lambda\left(W_{T^{\prime}}^{C}-W_{T^{\prime}}^{M}\right)$. Moreover, under those conditions ${ }^{C} X_{t}^{C B}$ is always bigger than ${ }^{M} X_{t}^{C B}$. It then follows that $\lambda\left(W_{T^{\prime}}^{C}-W_{T^{\prime}}^{M}\right)$, given by $\lambda\left[\left(f-R^{C} S_{t}^{C B}\right){ }^{C} X_{t}^{C B}-\left(f-R^{M} S_{t}^{C B}\right){ }^{M} X_{t}^{C B}\right]$, is always positive, and so is $L^{M}(\bar{S}, \lambda)-L^{C}(\bar{S}, \lambda)$. In other words, if $\lambda$ is "small", or if there is no trade-off between wealth-preservation and policy motives in selecting the optimal $X_{t}^{C B}$, the $\mathrm{CB}$ is always better off when dealers compete for the incoming trade. Instead, if such a trade-off exists and affects significantly the CB's decision process, then $L^{C}(\bar{S}, \lambda)>L^{M}(\bar{S}, \lambda)$ for some parametrizations of the model, as in the case of $E M_{t}<0$.

Uncertainty about $\bar{S}, f$, and $\lambda$ : optimality constraints. As suggested in Section 4.1 , the parameters of our model need to satisfy some rationality constraints to ensure that the intervention choices of Eqs. (28) and (29) are indeed optimal for the informed and uninformed CB. Let's start with the informed CB. The following set of constraints (Participation Constraints, $\mathrm{PC})$ guarantees that its optimal intervention schedule is different from zero, and that the signs of Eq. (28) are optimal:

$$
\begin{array}{lll}
\left(\bar{S}_{H}-\frac{E\left[f \mid I_{t}\right]}{R}\right)+\pi X^{*}+\frac{\lambda}{2 \pi L}\left(f_{H}-R S_{t}^{C B}\right)>0 & \text { PC }-\left(\bar{S}_{H}, f_{H}\right) \\
\left(\bar{S}_{L}-\frac{E\left[f \mid I_{t}\right]}{R}\right)+\pi X^{*}+\frac{\lambda}{2 \pi L}\left(f_{L}-R S_{t}^{C B}\right)<0 & \text { PC }-\left(\bar{S}_{L}, f_{L}\right)
\end{array}
$$




$$
\begin{array}{ll}
\left(\bar{S}_{H}-\frac{E\left[f \mid I_{t}\right]}{R}\right)+\pi X^{*}+\frac{\lambda}{2 \pi L}\left(f_{L}-R S_{t}^{C B}\right)>0 & \text { PC- }\left(\bar{S}_{H}, f_{L}\right) \\
\left(\bar{S}_{L}-\frac{E\left[f \mid I_{t}\right]}{R}\right)+\pi X^{*}+\frac{\lambda}{2 \pi L}\left(f_{H}-R S_{t}^{C B}\right)<0, & \text { PC- }\left(\bar{S}_{L}, f_{H}\right)
\end{array}
$$

where $E\left[f \mid I_{t}\right]$ is investors' prior about $f$. If we assume that the NFV of buying GBP assets is positive (negative) for $f_{H}\left(f_{L}\right)$ and that $\lambda$ is small enough, although not insignificant, for policy (speculation) motives to prevail in determining $X_{t}^{C B}$ 's sign (magnitude), those constraints are clearly satisfied for any set of parameters' values such that $\bar{S}_{H}$ is high enough and $\bar{S}_{L}$ is low enough with respect to $S_{t}^{*, l=0}: \bar{S}_{L}<<\frac{E\left[f \mid I_{\mathrm{t}}\right]}{B_{B}}-\pi X^{*}<<\bar{S}_{H}$. We also need to ensure that both ${ }_{B} X_{t}^{C B}>{ }_{S} X_{t}^{C B}>0$ and ${ }_{B} X_{t}^{C B}<{ }_{S} X_{t}^{C B}<0$, i.e., that the optimal schedule of Eq. (28) is compatible with the incentives implying from $L(\bar{S}, \lambda)$. It can be shown, however, that this is always the case for all possible sets of nature's draws for $f$ and $\bar{S}$, given the above assumptions about $\lambda, \bar{S}$, and the sign of $\left(f-R S_{t}^{C B}\right)$. For example, it is clear that when the pair $\left(f_{H}, \bar{S}_{H}\right)$ is true, the loss function resulting from Eq. (28), $L\left(\bar{S}_{H}, \lambda, f_{H, B} X_{t}^{C B}>0, S_{t}^{C B}\left({ }_{B} X_{t}^{C B}\right)\right)$, is lower than $L\left(\bar{S}_{H}, \lambda, f_{H},_{S} X_{t}^{C B}>0, S_{t}^{C B}\left({ }_{S} X_{t}^{C B}\right)\right)$ if the policy motive is sufficiently important (e.g. for small values of $\lambda$, or for an ambitious target $\left.\bar{S}_{H}\right)$ and $f_{H}$ is such that $\left(f_{H}-R S_{t}^{C B}\right)>0$. Along the same line, if the pair $\left(f_{L}, \bar{S}_{H}\right)$ is true, then $L\left(\bar{S}_{H}, \lambda, f_{L, S} X_{t}^{C B}>0, S_{t}^{C B}\left({ }_{S} X_{t}^{C B}\right)\right)$ is lower than $L\left(\bar{S}_{H}, \lambda, f_{L}, S X_{t}^{C B}<0, S_{t}^{C B}\left({ }_{S} X_{t}^{C B}\right)\right)$. Indeed, the gain ensuing from selling GBP, weighted by the small $\lambda$, is by assumption not big enough to compensate the informed CB for the loss stemming from a greater $\left[S_{t}^{*}-\bar{S}_{H}\right]^{2}$. Now, we turn our attention to the uninformed CB. We need to impose some restrictions on its beliefs about $f$ such that it is always true that ${ }^{U} X_{t}^{C B}>0$ for $\bar{S}_{H}$ and ${ }^{U} X_{t}^{C B}<0$ for $\bar{S}_{L}$. Hence, we need that, for the above conditions on $\bar{S}$, the following two PCs:

$$
\begin{array}{ll}
\left(\bar{S}_{H}-\frac{E\left[f \mid I_{t}\right]}{R}\right)+\pi X^{*}+\frac{\lambda}{2 \pi L}\left(E\left[f \mid \mathbf{z}_{t}^{U}\right]-R S_{t}^{C B}\right)>0 & \text { PC- }\left(\bar{S}_{H}, E\left[f \mid \mathbf{z}_{t}^{U}\right]\right) \\
\left(\bar{S}_{L}-\frac{E\left[f \mid I_{t}\right]}{R}\right)+\pi X^{*}+\frac{\lambda}{2 \pi L}\left(E\left[f \mid \mathbf{z}_{t}^{U}\right]-R S_{t}^{C B}\right)<0 & \text { PC- }\left(\bar{S}_{L}, E\left[f \mid \mathbf{z}_{t}^{U}\right]\right)
\end{array}
$$

are satisfied. If $f_{L} \leq E\left[f \mid \mathbf{z}_{t}^{U}\right] \leq f_{H}$, then a small enough value for $\lambda$ again ensures that, for each true $\bar{S}$, it is optimal for the uninformed CB to intervene in the direction postulated by Eq. (29). Finally, it is easy to verify that the pricing schedule of Eq. (30) is sufficient to ensure that mimicking is better than intervening with the amount implied by $L\left(\bar{S}, E\left[f \mid \mathbf{z}_{t}^{U}\right], S_{t}^{C B}=0\right.$ or $\left.\infty\right)$. We have in fact assumed that $\lambda$ is not small enough to make the wealth-preservation motive irrelevant in the monetary authority's loss function. Thus, given the selected order $\operatorname{sign}{ }^{U} X_{t}^{C B}>0$ $\left({ }^{U} X_{t}^{C B}<0\right)$ implying from $\bar{S}_{H}\left(\bar{S}_{L}\right)$, any order different from ${ }_{S} X_{t}^{C B}>0\left({ }_{S} X_{t}^{C B}<0\right)$ is going to be transacted at a very high (low) price, thus reducing (increasing) the NFV of investing in GBP-denominated bonds with respect to the pooling scenario, and in both cases pushing the corresponding loss function higher than if the uninformed CB had instead mimicked the informed $\mathrm{CB}$. In other words, given that wealth-preservation matters, the uninformed $\mathrm{CB}$ will always be worse off paying an extremely big price to buy GBP or receiving an extremely low price when 
selling GBP if $\lambda$ is not small enough to make the size of its future wealth irrelevant in the loss function $L\left(\bar{S}, E\left[f \mid \mathbf{z}_{t}^{U}\right]\right)$.

Proof of Proposition 6. As already mentioned in Section 4.1, under the assumption that $\left(f-R S_{t}^{C B}\right)$ is positive for $f_{H}$ and negative for $f_{L}, \bar{S}$ controls the sign of the intervention, while $f$ (or $E\left[f \mid \mathbf{Z}_{t}^{U}\right]$ ) explains its magnitude. Hence, the sign of the CB order is always fully revealing to the dealers of the true $\bar{S}$. It is also clear from Eqs. (28) and (29) that the arrival of big positive and negative interventions induces a full revelation of the true CB type to the MMs, as there is in fact one and one type only of informed CB that would optimally trade each of those order signs and sizes. When instead a small positive (negative) order is observed, the possibility that it is an uninformed CB to submit it induces just a partial revision of the dealers' prior probabilities for $f$ and $\bar{S}$. We assumed in Section 4.1 that the CB is informed about $f$ with probability $v$, and about $\bar{S}$ with probability 1 . By Bayes' Rule, it follows that, for $\xi_{H}=\operatorname{Pr}\left(X_{t}^{C B} \mid f_{H}, \bar{S}\right)$, we have:

$$
p_{f, t}=\frac{q_{f, t} \xi_{H}}{q_{f, t} \xi_{H}+\left(1-q_{f, t}\right) \xi_{L}} .
$$

Using Eqs. (28) and (29), and the fact that the sign of $X_{t}^{C B}$ is revealing of the true $\bar{S}$ to the dealers, we can calculate the conditional probability of arrival of each of the four possible orders. For example, if ${ }_{S} X_{t}^{C B}>0$ arrives, and consequently $\bar{S}_{H}$ is revealed to the MMs, then $\xi_{H}=\operatorname{Pr}\left(X_{t}^{C B} \mid f_{H}, \bar{S}_{H}\right)=(1-v)$ because just the uninformed CB would intervene with that magnitude for the pair $\left(f_{H}, \bar{S}_{H}\right)$. Thus, we have that $\xi_{H}=1$ and $\xi_{L}=0$ if ${ }_{B} X_{t}^{C B}>0$ arrives (and $\left.\bar{S}=\bar{S}_{H}\right), \xi_{H}=(1-v)$ and $\xi_{L}=v$ if ${ }_{S} X_{t}^{C B}>0$ arrives (and $\left.\bar{S}=\bar{S}_{H}\right), \xi_{H}=v$ and $\xi_{L}=(1-v)$ if ${ }_{S} X_{t}^{C B}<0$ arrives (and $\bar{S}=\bar{S}_{L}$ ), and that $\xi_{H}=0$ and $\xi_{L}=1$ if ${ }_{B} X_{t}^{C B}<0$ arrives (and $\left.\bar{S}=\bar{S}_{L}\right)$. It then follows that, when $\bar{S}=\bar{S}_{H}, p_{f, t}=1$ if $X_{t}^{C B}={ }_{B} X_{t}^{C B}>0$, but that $p_{f, t}$ is given by Eq. (34) if $X_{t}^{C B}={ }_{S} X_{t}^{C B}>0$. However, when $\bar{S}=\bar{S}_{L}, p_{f, t}=0$ if $X_{t}^{C B}={ }_{B} X_{t}^{C B}<0$, but that $p_{f, t}$ is given by Eq. (35) if $X_{t}^{C B}={ }_{S} X_{t}^{C B}<0$. Finally, we derive dealers' beliefs about $l$. Small $X_{t}^{C B}$ are not fully revealing about $f$ because of mimicking behavior by the uninformed CB $(v<1)$, hence we assume MMs use Bayes' Rule to revise their beliefs about $l$. When instead a big intervention occurs, the dealers know what the true $\bar{S}$ and $f$ are. Thus, they use that information to learn what $l$ is (and their posterior becomes $p_{l, t}=l$ ) from the observed order sign, as ${ }_{B} X_{t}^{C B}$ is function of $l$ as well. The new prior $q_{l, t+1}=p_{l, t}$ is still subject to change due to the incoming order flow, because $\operatorname{Var}\left[l \mid M_{t}\right]$, a proxy for CB credibility, is still positive. When an investors' order $\left(X_{i, t}\right)$ or a small CB order $\left({ }_{S} X_{t}^{C B}\right)$ arrives, from Bayes' Rule the resulting conditional posterior distribution of $l$ is given by

$$
f_{M_{\mathrm{t}}, X_{\mathrm{i}, \mathrm{t}}}(l)=\frac{(1-l) f_{M_{\mathrm{t}}}(l)}{\int_{0}^{1}(1-l) f_{M_{\mathrm{t}}}(l) d l}
$$

or by

$$
f_{M_{\mathrm{t}, \mathrm{S}} X_{\mathrm{t}}^{\mathrm{CB}}}(l)=\frac{l f_{M_{\mathrm{t}}}(l)}{\int_{0}^{1} l f_{M_{\mathrm{t}}}(l) d l},
$$


respectively. As in Saar (2000a), it is then easy to show that, if $X_{i, t}$ arrives,

$$
\begin{aligned}
p_{l, t} & =\int_{0}^{1} l f_{M_{\mathrm{t}}, X_{\mathrm{i}, \mathrm{t}}}(l) d l=\frac{\int_{0}^{1} l(1-l) f_{M_{\mathrm{t}}}(l) d l}{\int_{0}^{1}(1-l) f_{M_{\mathrm{t}}}(l) d l}= \\
& =\frac{E\left[l \mid M_{t}\right]-E\left[l^{2} \mid M_{t}\right]}{1-E\left[l \mid M_{t}\right]}=E\left[l \mid M_{t}\right]-\frac{\operatorname{Var}\left[l \mid M_{t}\right]}{1-E\left[l \mid M_{t}\right]}
\end{aligned}
$$

while, if ${ }_{S} X_{t}^{C B}$ arrives,

$$
\begin{aligned}
p_{l, t} & =\int_{0}^{1} l f_{M_{\mathrm{t}, \mathrm{s}} X_{\mathrm{t}}^{\mathrm{CB}}}(l) d l=\frac{\int_{0}^{1} l^{2} f_{M_{\mathrm{t}}}(l) d l}{\int_{0}^{1} l f_{M_{\mathrm{t}}}(l) d l}= \\
& =\frac{E\left[l^{2} \mid M_{t}\right]}{E\left[l \mid M_{t}\right]}=E\left[l \mid M_{t}\right]+\frac{\operatorname{Var}\left[l \mid M_{t}\right]}{E\left[l \mid M_{t}\right]} .
\end{aligned}
$$

Proof of Proposition 7. Investors update their beliefs about $p_{f}$ when they observe the sign of $\Delta S_{i, t+1}$ following the CB intervention. Investors are by assumption unaware of any of dealers' past priors and new posteriors for $\bar{S}, f$, or $l$, hence cannot extrapolate any of them from the last transaction price. However, they know that CBs are uninformed with probability $1-v$, that interventions produce an impact on quotes just if unexpected (after controlling for the impact of $X_{t}^{C B}$ and $X_{i, t}$ on $q_{l, t}$ ), and that, if an intervention hits the dealers' screens, the resulting transaction is big with probability $\psi$. Because the CB, when informed, acts according to Eq. (28), and because, if uninformed, the CB instead mimics the moves of the informed monetary authority, it then follows that $\operatorname{Pr}\left\{\Delta S_{i, t+1}>0 \mid f_{H}\right\}=\psi+(1-\psi) v, \operatorname{Pr}\left\{\Delta S_{i, t+1}<0 \mid f_{H}\right\}=(1-\psi)(1-v)$, $\operatorname{Pr}\left\{\Delta S_{i, t+1}>0 \mid f_{L}\right\}=(1-\psi)(1-v)$, and $\operatorname{Pr}\left\{\Delta S_{i, t+1}<0 \mid f_{L}\right\}=\psi+(1-\psi) v$. For example, given that an intervention actually occurred, a $\Delta S_{i, t+1}>0$ can arise from an unexpectedly big positive CB order (revealing $\bar{S}$ to the dealers and inducing them to revise upward their beliefs about $f$ ) with probability $\psi$, or from an unexpectedly small negative order (inducing the dealers to revise upward a previously small prior about $f$ ) with probability $1-\psi$. In the first case, ${ }_{B} X_{t}^{C B}>0$ may originate just from an informed $\mathrm{CB}$ aware of $f=f_{H}$. In the second case, ${ }_{S} X_{t}^{C B}<0$ may be the result of the actions of a CB informed about $f_{H}$ just with probability $v$, as an uninformed CB finds optimal to intervene with a small negative order even if $f=f_{L}$. Finally, for simplicity, we impose that $\operatorname{Var}\left[l \mid M_{t}\right]$ is small enough so that, following the arrival of the uninformative order $X_{i, t+1}$ and along the lines of Eq. (33), $q_{l, t+2}$ does not decline "too much," i.e., $\left|q_{l, t+2}-q_{l, t+1}\right|$ is not "too big," and investors do not need to control for the part of the quote's revision that is due to dealers' new beliefs about $l$, when they update their prior ${ }^{I} q_{f, 0}$. Indeed, this assumption allows us to avoid the scenario in which CB's credibility is so low (i.e., $\left|q_{l, t+2}-q_{l, t+1}\right|$ is so big) to more than compensate the effect of the dealers' new posteriors $p_{f, t}, p_{l, t}$, and $p_{\bar{S}, t}$ on the transaction price change $\Delta S_{i, t+1}$, hence so big to induce our investors to false inference on $f$. Consequently, by Bayes' Rule, if for example $\Delta S_{i, t+1}>0$ is observed, we have that

$$
{ }^{I} p_{f, t+1}=\frac{{ }^{I} q_{f, t} \operatorname{Pr}\left\{\Delta S_{i, t+1}>0 \mid f_{H}\right\}}{{ }^{I} q_{f, t} \operatorname{Pr}\left\{\Delta S_{i, t+1}>0 \mid f_{H}\right\}+\left(1-{ }^{I} q_{f, t}\right) \operatorname{Pr}\left\{\Delta S_{i, t+1}>0 \mid f_{L}\right\}} .
$$

The expression for ${ }^{I} p_{f, t+1}$ when $\Delta S_{i, t+1}<0$ is similarly obtained. Eq. (36) then follows. 


\section{References}

[1] Amihud, Y., Mendelson, H. (1980). Dealership Market: Market-Making with Inventory. Journal of Financial Economics, 8, pp. 31-53.

[2] Bank for International Settlements (1999). Central Bank Survey of Foreign Exchange and Derivatives Market Activity 1998. Available at http://www.bis.org.

[3] Bhattacharya, U., Weller, P. (1997). The Advantage of Hiding One's Hand: Speculation and Central Bank Intervention in the Foreign Exchange Market. Journal of Monetary Economics, 39, pp. 251-277.

[4] Bossaerts, P., Hillion, P. (1991). Market Microstructure Effects of Government Intervention in the Foreign Exchange Market. Review of Financial Studies, 4, pp. 513-541.

[5] Branson, W. H. (1983). Macroeconomic Determinants of Real Exchange Risk. In Managing Foreign Exchange Risk, Ed. R. J. Herring, Cambridge University Press, Cambridge.

[6] Branson, W. H. (1984). A Model of Exchange Rate Determination with Policy Reaction: Evidence from Monthly Data. In Contemporary Macroeconomic Modelling, Eds. P. Malgrange and P. Muet, Basil Blackwell, Oxford, U.K.

[7] Brock, W., Kleidon, A. (1992). Periodic Market Closure and Trading Volume: a Model of Intraday Bids and Asks. Journal of Economic Dynamics and Control, 16, pp. 451-489.

[8] Brown, S. (2000). Hedge Funds: Omniscient or Just Plain Wrong. Keynote Address for the PACAP/FMA Meeting, Melbourne, Australia.

[9] Cadenillas, A., Zapatero, F. (1999). Optimal Central Bank Intervention in the Foreign Exchange Market. Journal of Economic Theory, 87, pp.218-242.

[10] Cadenillas, A., Zapatero, F. (2000). Classical and Impulse Stochastic Control of the Exchange Rate Using Interest Rate and Reserves. Mathematical Finance, 10, pp. 141-156.

[11] Chancellor, E. (2000). Devil Take the Hindmost: a History of Financial Speculation. Farrar, Straus \& Giroux Inc., New York.

[12] Dominguez, K. M. (1999). The Market Microstructure of Central Bank Intervention. NBER Working Paper Series No. 7337, Cambridge.

[13] Dominguez, K. M., Frankel, J. A. (1993 a). Does Foreign Exchange Intervention Matter? The Portfolio Effect. American Economic Review, 83, pp. 1356-1369.

[14] Dominguez, K. M., Frankel, J. A. (1993 b). Does Foreign Exchange Intervention Work? Institute for International Economics, Washington.

[15] Eaton, J., Turnowsky, S. J. (1984). The Forward Exchange Market, Speculation, and Exchange Market Intervention. Quarterly Journal of Economics, 99, pp. 45-70. 
[16] Easley, D., O'Hara, M. (1987). Price, Trade Size, and Information in Securities Markets. Journal of Financial Economics, 19, pp. 69-90.

[17] Easley, D., O'Hara, M. (1991). Order Form and Information in Securities Markets. Journal of Finance, 46, pp. 905-928.

[18] Easley, D., O'Hara, M. (1992). Time and the Process of Security Price Adjustment. Journal of Finance, 47, pp. 577-606.

[19] Edison, H. J. (1993). The Effectiveness of Central Bank Intervention: a Survey of Post-1982 Literature. Essays in International Finance, Princeton University Press, Princeton.

[20] Evans, M., Lyons, R. K. (1999). Order Flow and Exchange Rate Dynamics. NBER Working Paper Series No. 7317, Cambridge.

[21] Evans, M., Lyons, R. K. (2001). Portfolio Balance, Price Impact, and Secret Intervention. Working Paper 8356, NBER.

[22] Fischer, A. M., Zurlinden, M. (1999). Exchange Rate Effects of Central Bank Interventions: an Analysis of Transaction Prices. Economic Journal, 109, pp. 662-676.

[23] Foster, F. D., Viswanathan, S. (1996). Strategic Trading When Agents Forecast the Forecasts of Others. Journal of Finance, 51, pp. 1437-1478.

[24] Friedman, M. (1953). The Case for Flexible Exchange Rates. In Essays in Positive Economics, University of Chicago Press, Chicago.

[25] Garman, M. B. (1976). Market Microstructure. Journal of Financial Economics, 3, pp. $257-275$.

[26] Glosten, L., Milgrom, P. (1985). Bid, Ask and Transaction Prices in a Specialist Market with Heterogeneously Informed Traders. Journal of Financial Economics, 14, pp. 71-100.

[27] Goodhart, C., Hesse, T. (1991). Central Bank Forex Intervention Assessed in Continuous Time. London School of Economics Financial Markets Group Discussion Paper No. 123.

[28] Grossman, S. J., Stiglitz, J. E. (1980). On the Impossibility of Informationally Efficient Markets. American Economic Review, 70, pp. 393-408.

[29] Jurgensen, P. (1983). Report of the Working Group on Exchange Market Intervention. U.S. Treasury Department, Washington.

[30] Kaminsky, G. L., Lewis, K. (1996). Does Foreign Exchange Intervention Signal Future Monetary Policy? Journal of Monetary Economics, 37, pp. 285-312.

[31] Kyle, A. S. (1985). Continuous Auctions and Insider Trading. Econometrica, 53, pp. 13151335.

[32] Lewis, K. (1995). Occasional Intervention to Target Rates. American Economic Review, 85, pp. 691-715. 
[33] Lyons, R. K. (1995). Tests of Microstructural Hypotheses in the Foreign Exchange Market. Journal of Financial Economics, pp. 321-351.

[34] Lyons, R. K. (1997). A Simultaneous Trade Model of the Foreign Exchange Hot Potato. Journal of International Economics, pp. 275-298.

[35] Lyons, R. K. (2001). The Microstructure Approach to Exchange Rates. MIT Press, Cambridge.

[36] Madhavan, A. (1992). Trading Mechanisms in Securities Markets. Journal of Finance, 47, pp. 607-641.

[37] Madhavan, A. (2000). Market Microstructure: A Survey. Journal of Financial Markets, 3, pp. 205-258.

[38] Madrigal, V. (1996). Non-Fundamental Speculation. Journal of Finance, 51, pp. 553-578.

[39] Miller, M. H. (1998). Asia's Currency Crisis: Problems and Prescriptions. Speech given to the Asia Society in Hong Kong.

[40] Moloney, T. (2000). G7 Intervention - An Historical Perspective. Deutsche Bank Global Markets Research, New York.

[41] Mussa, M., (1981). The Role of Official Intervention. Group of Thirty, New York.

[42] Naranjo, A., Nimalendran, M. (2000). Government Intervention and Adverse Selection Costs in Foreign Exchange Markets. Review of Financial Studies, 13, pp. 453-477.

[43] Neely, C. J. (2000). Are Changes in Foreign Exchange Reserves Well Correlated with Official Intervention? Federal Reserve Bank of St. Louis Review, St. Louis.

[44] O’Hara, M. (1995). Market Microstructure Theory. Blackwell, Cambridge.

[45] Pasquariello, P. (2000). The Microstructure of Currency Markets: an Empirical Model of Intraday Return and Bid-Ask Spread Behavior. Working Paper, Stern School of Business, New York University.

[46] Pasquariello, P. (2002). Informative Trading or Just Noise? An Analysis of Currency Returns, Market Liquidity, and Transaction Costs in Proximity of Central Bank Interventions. Working Paper, Stern School of Business, New York University.

[47] Payne, R., Vitale, P. (2001). A Transaction Level Study of the Effects of Central Bank Intervention on Exchange Rates. Discussion Paper 3085, CEPR.

[48] Peiers, B. (1997). Informed Traders, Intervention and Price Leadership: a Deeper View of the Microstructure of the Foreign Exchange Market. Journal of Finance, 52, pp. 1589-1614.

[49] Persson, T., Tabellini, G. (1990). Macroeconomic Policy, Credibility and Politics. Harwood Academic Publishers, London. 
[50] Rogoff, K. (1985). The Optimal Degree of Commitment to an Intermediate Monetary Target. Quarterly Journal of Economics, 100, pp. 1169-1189.

[51] Saar, G. (2000a). Demand Uncertainty and the Information Content of the Order Flow. Working Paper, Stern School of Business, New York University.

[52] Saar, G. (2000b). Prices and Spreads in Sequential Markets with Information Imperfections. Working Paper, Stern School of Business, New York University.

[53] Sarno, L., Taylor, M. P. (2001). Official Intervention in the Foreign Exchange Market: Is it Effective, and if So, How Does it Work? Journal of Economic Literature, 39, pp. 839-868.

[54] Stein, J. C. (1989). Cheap Talk and the Fed: a Theory of Imprecise Policy Announcements. American Economic Review, 79, pp. 32-42.

[55] Stoll, H. R. (1978). The Supply of Dealer Services in Securities Markets. Journal of Finance, 33, pp. 1133-1151.

[56] Sweeney, R. J. (1997). Do Central Banks Lose on Foreign-Exchange Intervention? A Review Article. Journal of Banking and Finance, 21, pp. 1667-1684.

[57] Taylor, D. (1982). Official Intervention in the Foreign Exchange Market, or, Bet Against the Central Bank. Journal of Political Economy, 90, pp. 356-368.

[58] Taylor, M. P. (1995). The Economics of Exchange Rates. Journal of Economic Literature, 33, pp. 13-47.

[59] Varian, H. (1985). Divergence of Opinion in Complete Markets: a Note. Journal of Finance, 40, pp. 309-317.

[60] Vitale, P. (1999). Sterilised Foreign Exchange Intervention in the Foreign Exchange Market. Journal of International Economics, 49, pp. 245-267.

[61] Walsh, C. E. (1995). Optimal Contracts for Central Bankers. American Economic Review, 85, pp. 150-167. 
Figure 1. Intervention with a monopolist dealer and full information

Comparative statics: monopolist dealer, full information, and CB chasing the trend. Exchange rate $S_{t}$ on right axis, $\Delta S_{i, t}$ on left axis. Model parametrization: $\alpha=1, R=1.05, \sigma_{F}^{2}=0.0045, \bar{X}_{1}=15, \bar{X}_{2}=5$, $q=0.5, f=1.7, \bar{S}=1.58, \lambda=0.00001$. The resulting $S_{t}^{*, l=0}=\frac{f}{R}-\pi X^{*}=1.5761$.

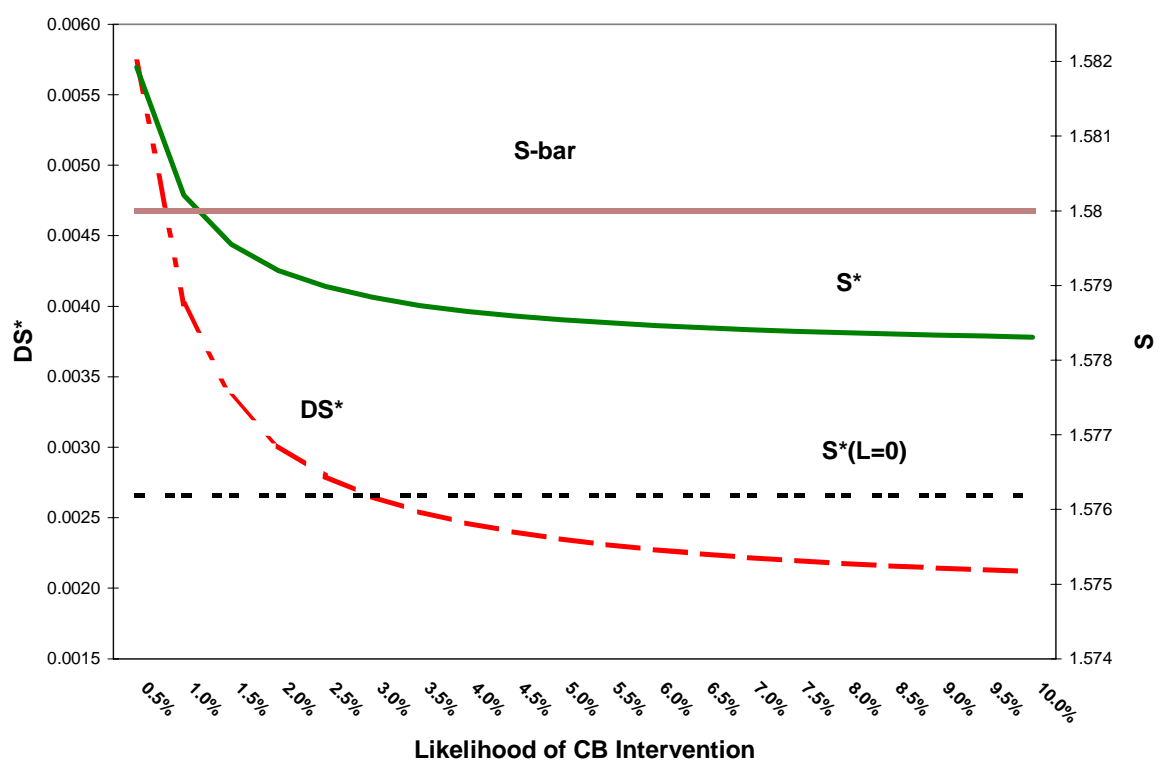

Figure 2. Intervention with competitive dealers and full information

Comparative statics: competitive dealers, and full information. Units of foreign currency on right axis, spread on left axis. Model parametrization: $\alpha=1, R=1.05, \sigma_{F}^{2}=0.025, \bar{X}_{1}=15, \bar{X}_{2}=5, q=0.5, f=1.7$, $\bar{S}_{a}=1.45, \bar{S}_{b}=1.35, \lambda=0.00001, S_{t}^{C B}=\bar{S}$. The resulting $S_{t}^{*, l=0}=\frac{f}{R}-\pi X^{*}=1.3810$.

a) $\mathrm{CB}$ chasing the trend

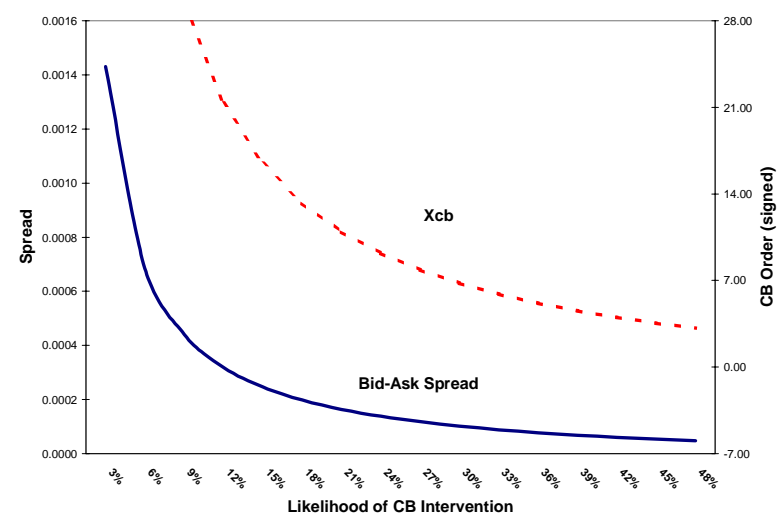

b) CB leaning against the wind

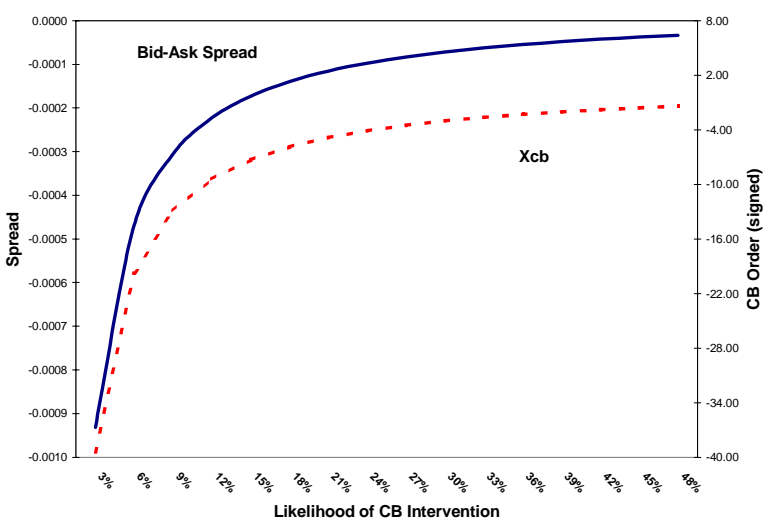


Figure 3. Intervention with competitive dealers and full information: sensitivity analysis

Comparative statics: competitive dealers, full information, and CB chasing the trend. (a) Dollar revenues on right axis, spread on left axis. (b) Units of foreign currency on right axis, spread on left axis. Model parametrization: $\alpha=1, R=1.05, \sigma_{F}^{2}=0.025, \bar{X}_{1}=15, \bar{X}_{2}=5, q=0.5, f=1.7, \bar{S}_{a}=1.45, \lambda=$ $0.00001, l=0.03$. The resulting $S_{t}^{*, l=0}=\frac{f}{R}-\pi X^{*}=1.3810$.

a) Spread versus $\frac{\bar{X}_{1}}{\bar{X}_{2}}$

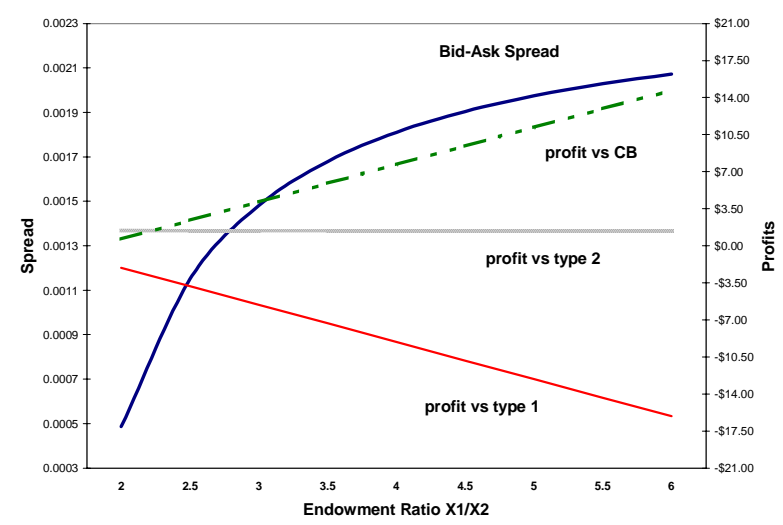

b) Spread and $X_{t}^{C B}$ versus $S_{t}^{C B}$

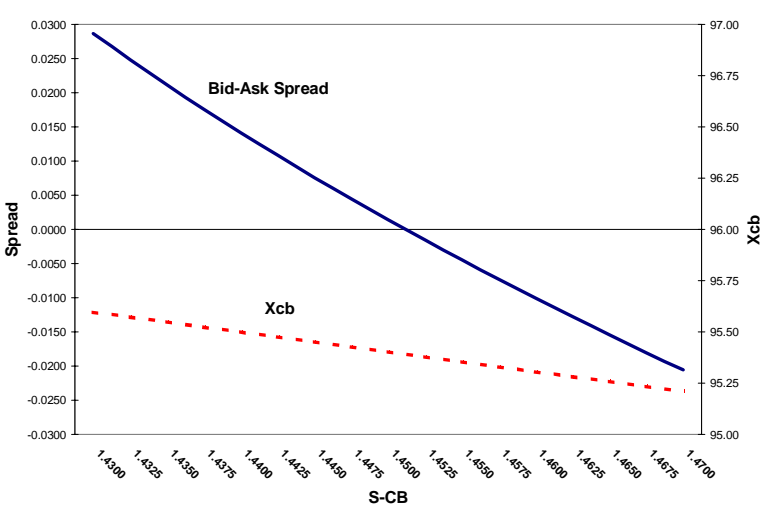

Figure 4. Effectiveness of intervention with full information

Comparative statics: full information, and CB chasing the trend. Exchange rate $S_{t}$ on right axis, GBP amounts on left axis. Model parametrization: $\alpha=1, R=1.05, \sigma_{F}^{2}=0.025, \bar{X}_{1}=15, \bar{X}_{2}=5, q=0.5$, $f=1.7, \bar{S}=1.45, \lambda=0.00001$. The resulting $S_{t}^{*, l=0}=\frac{f}{R}-\pi X^{*}=1.3810$.

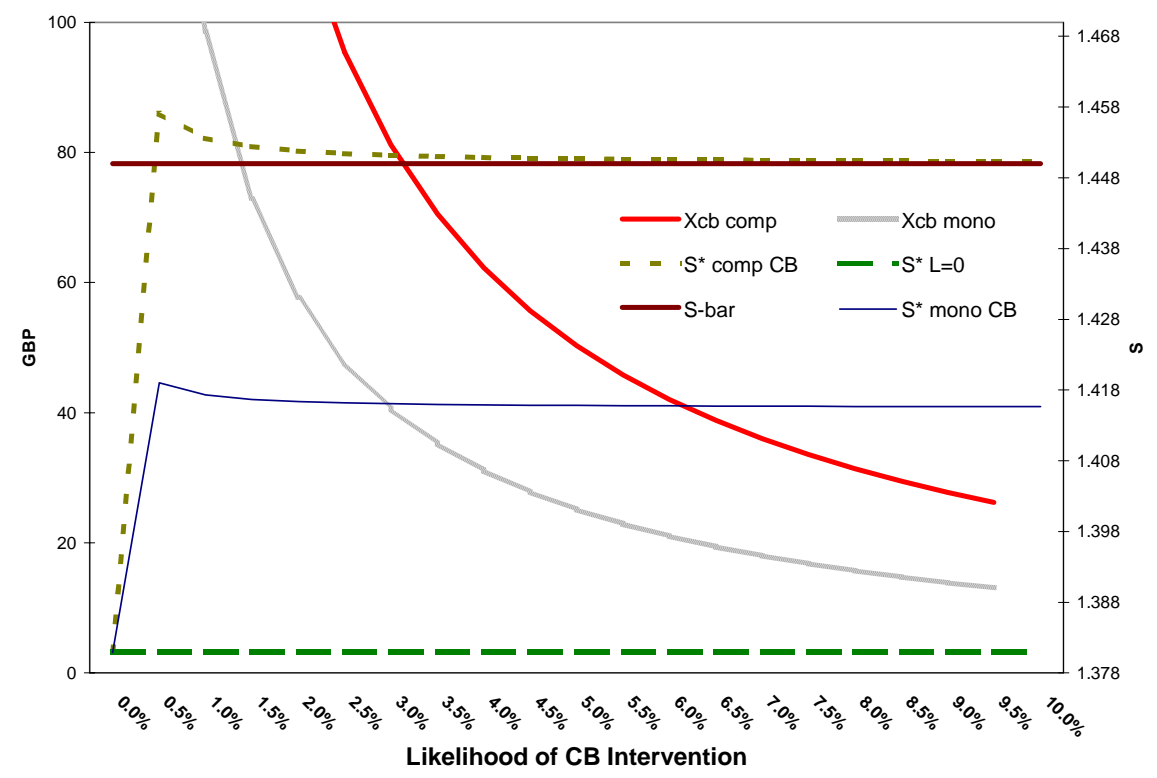




\section{Figure 5. Intervention with information asymmetry}

Monopolist currency dealer (a) and competitive currency dealers (b): CB leaning against the wind with ${ }_{B} X_{t_{6}}^{C B}<0$. Expected exchange rate $S_{t}^{*}$ on left axis (dark continuous line), transaction prices $S_{i, t}$ on left axis (light continuous line), proportional spread $P S_{t_{\mathrm{n}}}$ on right axis (dotted line). Model parametrization: $\alpha=1$, $R=1.05, \sigma_{F}^{2}=0.015, \bar{X}_{1}=12, \bar{X}_{2}=1, q=0.25, f_{H}=1.6, f_{L}=1.45, \bar{S}_{H}=1.5, \bar{S}_{L}=1.31$, $\lambda=0.001$. Market's beliefs: ${ }^{I} q_{f, 0}=0.75,{ }^{U} q_{f, 0}=0.5, q_{f, 0}=0.75, v=0.8, \psi=0.3, q_{\bar{S}, 0}=$ $q_{f, 0} \psi+\left(1-q_{f, 0}\right)(1-\psi)=0.40, E\left[\bar{S} \mid M_{0}\right]=1.3860$. The star symbol on the $\mathrm{x}$-axis indicates when the intervention occurs in the simulated sequence of events.

a) Monopolist MM

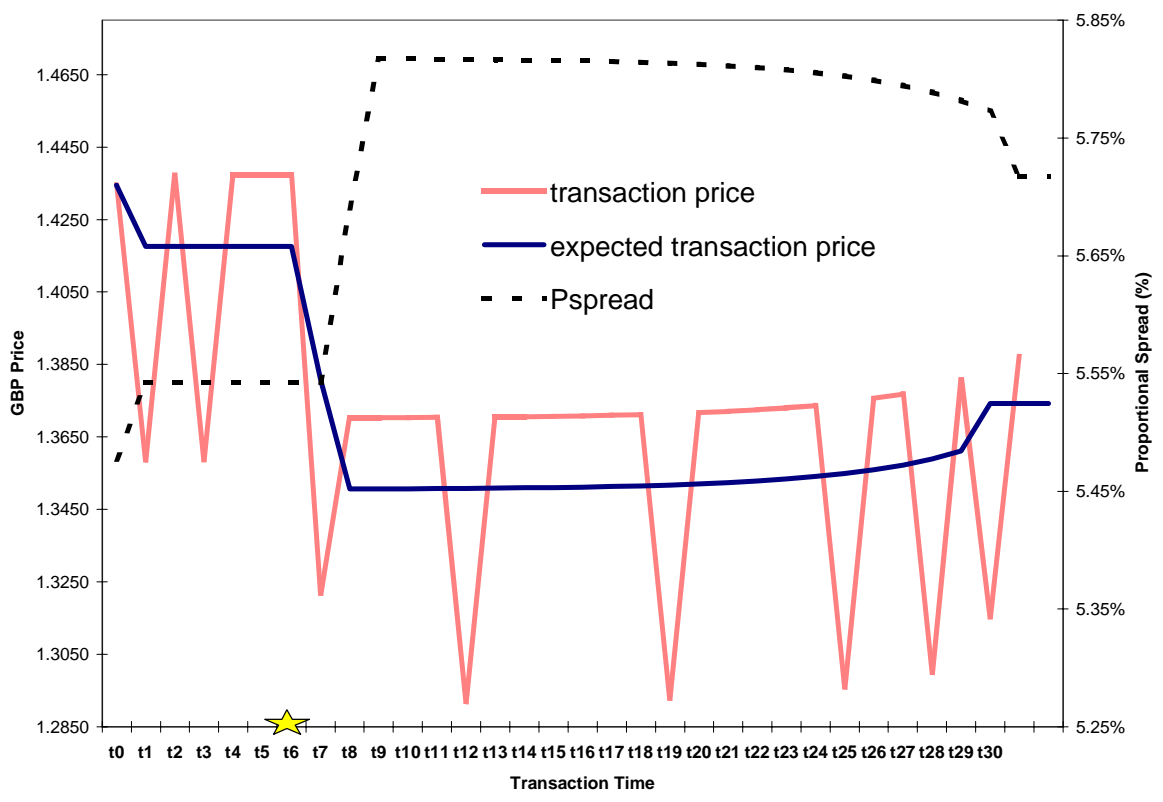

b) Competitive MMs

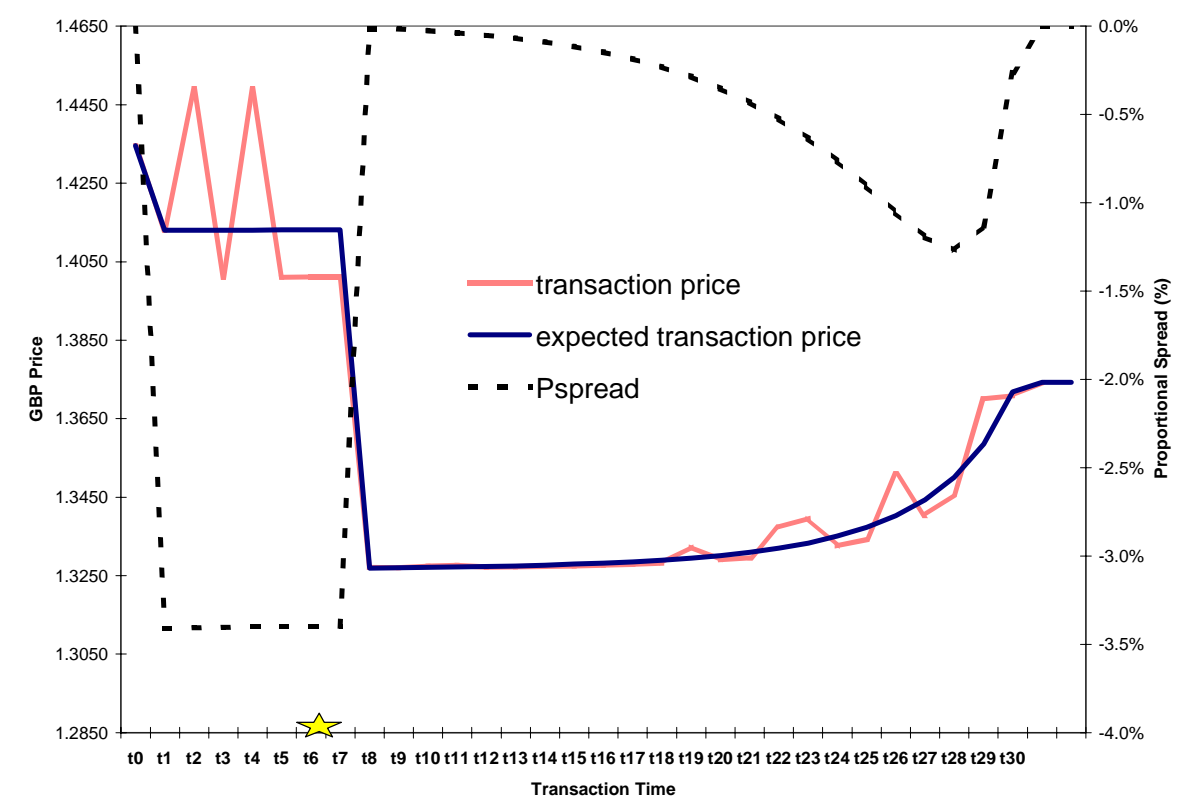

